\title{
THE MORPHOLOGY, SYNTAX, AND SEMANTICS OF ADVERBS IN CANTONESE
}

\author{
WONG LAI YIN
}

王麗 賢

M. PHIL. THESIS

THE UNIVERSITY OF HONG KONG

2002 


\title{
Abstract of thesis entitled \\ The Morphology, Syntax, and Semantics of Adverbs in Cantonese
}

\author{
submitted by \\ Wong Lai Yin \\ for the degree of Master of Philosophy \\ at the University of Hong Kong \\ in September 2002
}

This thesis reports on a study of adverbs in Cantonese as it is spoken in contemporary Hong Kong.

Previous studies of Cantonese adverbs have put particular emphasis on the grammatical meanings of a few individual adverbs, for instance, the adverb 先 sin1 (Cheng, 1990; Cai, 1995; Luke 2002; among others). The general properties of the adverb class as a whole have received relatively scant attention. In this study, the characteristics of Cantonese adverbs, and especially the morphologically, semantically and syntactically relevant aspects of these adverbs are investigated in greater depth. The search for general patterns has been given priority above the description of the peculiarities of each individual adverb.

Linguists have generally agreed that an adverb can serve as an adverbial in a sentence (see, for example, Quirk \& Greenbaum, 1973). Zhu Dexi (1982), in particular, provides the most insightful criterion in distinguishing adverb from other word classes. He proposes that an adverb can and can only function as adverbial, and never function as any other sentence constituent like subject and predicate. In the present study, only those words that can function only as adverbials according to Zhu's analysis are identified as adverbs. Particular attention is given to distinguishing adverbs from adjectives, time words (nouns) and conjunctions, which are commonly used as adverbials as well.

For the purpose of the present study, a collection, which should be fairly comprehensive, of 544 adverbs have been made. 242 of these have cognates in Mandarin whereas 302 are unique to Cantonese. An exploration of the form of these adverbs suggests that reduplication is the most salient characteristics especially in trisyllabic and polysyllabic adverbs. Cantonese adverbs are further 
divided into seven subcategories based on semantic and grammatical criteria; manner adverbs, among these seven subclasses, tend to have the greatest number of members. It was observed that Cantonese adverbs modify verb phrase, adjective phrase and noun phrase as well as modify on the clausal level as adverbials. The fact that adverb subclasses differ in the syntactic categories they modify clearly justifies the classification used in this study. Cantonese adverbs are further shown to exhibit their own peculiarities in syntactic properties as distinguished from English and Mandarin Chinese ones.

Further investigations in Cantonese adverbs should consider their productivity and distribution with respect to sentence types.

(379 words) 


\section{Contents}

Declaration

Acknowledgements

ii

Table of Contents

iii

List of Illustrations/Tables/Figures/Appendices

$v i$

Abbreviations

vii

\section{Chapter 1: Introduction}

1.1 Identification of Adverbs

1.2 Previous Analyses on Adverbs

1.3 Objectives of Present Research 6

1.4 Methodological Issues $\quad 7$

1.4.1 Identification Criteria 7

1.4.2 Collection of Data 8

1.4.2.1 Corpus 8

1.4.2.2 Dictionaries 9

$\begin{array}{ll}\text { 1.4.2.3 Mass Media } & 10\end{array}$

1.4.2.4 Native-speaker Introspection $\quad 11$

$\begin{array}{lll}\text { 1.4.3 Presentation of Data } & 11\end{array}$

1.4.3.1 Use of Parentheses and Slash 11

1.4.3.2 Translation of Cantonese Examples 11

1.4.4 Final Remarks 12

1.5 Organisation of Thesis 12

Chapter 2: Adverbs and Other Word Classes $\quad 14$

$\begin{array}{lll}2.1 & \text { Towards a Definition of Adverbs } & 14\end{array}$

2.2 As Distinguished from Adjectives, Nouns (Time Words) and Subordinate $\begin{array}{ll}\text { Conjunctions } & 18\end{array}$

2.2.1 Adverbs and Adjectives 18

$\begin{array}{ll}\text { 2.2.2 Adverbs and Nouns (Time Words) } & 19\end{array}$

$\begin{array}{ll}\text { 2.2.3 Adverbs and Conjunctions } & 19\end{array}$

$\begin{array}{lll}2.3 & \text { Summary } & 21\end{array}$ 
Chapter 3: Forms of Adverbs

3.1 Simple and Complex Adverbs $\quad 22$

3.1.1 Simple Adverbs 23

3.1.1.1 Monosyllabic Adverbs 23

3.1.1.2 Disyllabic Adverbs 24

3.1.1.3 Trisyllabic Adverbs $\quad 33$

3.1.1.4 Polysyllabic Adverbs $\quad 34$

3.1.2 Complex Adverbs $\quad 35$

3.1.2.1 Monosyllabic Adverbs 35

3.1.2.2 Disyllabic Adverbs $\quad 35$

$\begin{array}{lll}\text { 3.1.2.3 Trisyllabic Adverbs } & 37\end{array}$

3.1.2.4 Polysyllabic Adverbs $\quad 39$

$\begin{array}{lll}3.2 & \text { Recurrent Patternings of Adverbs } & 40\end{array}$

3.2.1 With Directional Complement 嚟 lai4 40

3.2.2 With Directional Complement 上soeng6 40

$\begin{array}{lll}3.2 .3 & \text { With Negation Adverb } & 40\end{array}$

3.2.4 With 中 zungl 41

3.2.5 With 腳 goek3 41

3.2.6 With 意 $j i 2 \quad 41$

3.2.7 With 然 jin4 41

3.2.8 With 之zil 41

3.2.9 AA + 聲 sengl $\quad 42$

3.2.10 A-唔 $m 4-\mathrm{A} \quad 42$

3.2.11 A-不batl-A 42

3.2.12 $\mathrm{V} / \mathrm{ADJ}+$ 㕷 $d i 1$

3.2.13 V+ 吓haa5 43

3.2.14 Verb + Object 43

3.2.15 $\quad \mathrm{ADJ}_{\mathrm{i}}+\mathrm{ADJ}_{\mathrm{i}}+$ 吔 dei2 43

3.2.16 親 can $1+$ Noun (denoting body part responsible for speech or actions) 44

3.2.17 Antonymous Combinations 44

3.3 Reduplicated Adverbs 44

3.3.1 AA Form 44

3.3.2 $\mathrm{AAB}$ Form $\quad 45$

3.3.3 ABA Form 46

$\begin{array}{lll}\text { 3.3.4 ABB Form } & 46\end{array}$

3.3.5 AABB Form 47

$\begin{array}{lll}\text { 3.3.6 } & \mathrm{ABAB} \text { Form } & 47\end{array}$

$\begin{array}{lll}\text { 3.3.7 } & \text { ABAC Form } & 47\end{array}$

$\begin{array}{lll}\text { 3.3.8 } & \mathrm{ABCB} \text { Form } & 48\end{array}$ 
3.4 Co-ordinate Pairs 48

$\begin{array}{ll}3.5 & \text { Antonym Pairs } \\ & 49\end{array}$

$\begin{array}{ll}3.6 \text { Summary } & 50\end{array}$

Chapter 4: Semantic Classification of Adverbs $\quad 51$

4.1 Introduction: Proposals for classification in the literature 51

4.1.1 English classification of adverbs 51

4.1.2 Chinese classification of adverbs 53

4.2 Conjunctive Adverb (C) 56

4.3 Degree Adverb (D) 57

4.4 Manner Adverb (M) 61

4.5 Modal Adverb (ML, MA, MV) 65

$\begin{array}{lll}4.6 & \text { Negation Adverb }(\mathrm{N}) & 69\end{array}$

$\begin{array}{lll}4.7 & \text { Scope Adverb (S) } & 71\end{array}$

4.8 Time Adverb (T) $\quad 75$

$\begin{array}{lll}4.9 & \text { Final Remarks } & 77\end{array}$

Chapter 5: Syntactic Functions of Adverbs $\quad 80$

5.1 Introduction $\quad 80$

5.2 Functions in a Phrase $\quad 82$

5.2.1 Modifier of Verb Phrase 82

5.2.2 Modifier of Adjective Phrase 86

5.2.3 Apparent Modifier of Prepositional Phrase 88

5.2.4 Modifier of Noun Phrase 90

5.3 Function in a Clause - Adverbial 93

5.4 Substantiation of Sub-classification of Adverbs 95

$\begin{array}{lll}5.5 & \text { Summary } & 97\end{array}$

Chapter 6: Conclusion and Further Research 98

6.1 Conclusions 98

6.2 Areas of Future Research 99

6.2.1 Productivity of Adverbs 99

6.2.2 Distribution of Adverbs (with respect to sentence types) 100

$\begin{array}{lll}6.3 \text { Final Remarks } & 100\end{array}$

$\begin{array}{lr}\text { Appendices } & 101\end{array}$

References $\quad 133$ 


\section{Illustrations}

\section{Figures}

\section{Figure 1}

$\begin{array}{ll}\text { A framework of classifying adverbs } & 16\end{array}$

Figure 2

$\begin{array}{ll}\text { Degree adverbs and degree scale } & 58\end{array}$

Figure 3

Syntactic and semantic subclassification of adverbs in Cantonese

96

\section{Tables}

\section{Table 1}

Simple and complex adverbs in Cantonese $\quad 50$

\section{Table 2}

The classification of adverbs in the Chinese literature

\section{Table 3}

Subclassification of adverbs in Cantonese and their grammatical meanings

79

\section{Table 4}

An illustration of different adverb sub-classes modifying different elements

96

\section{Appendices}

\section{Appendix 1}

A list of Cantonese adverbs

Appendix 2

Classification of adverbs by grammatical meanings 


\section{Abbreviations}

$\begin{array}{ll}\text { ADJ } & \text { Adjective } \\ \text { ASP } & \text { Aspect Marker } \\ \text { COMP } & \text { Verb Complement } \\ \text { EMPH } & \text { Expressing Emphasis } \\ \text { FP } & \text { Final Particle } \\ \text { IP } & \text { Initial Particle } \\ \text { LOC } & \text { Locative Marker e.g. 度 dou6 } \\ \text { M } & \text { Measure Word } \\ \text { MP } & \text { Medial Particle } \\ \text { PL } & \text { Plural Marker } \\ \text { POSS } & \text { Possessive Marker de in Mandarin Chinese } \\ \text { PREP } & \text { Preposition with No Obvious Meaning e.g. 將 zoengl } \\ \text { V } & \text { Verb }\end{array}$




\section{troduction}

This study deals with different grammatical aspects of adverbs in Cantonese. Cantonese grammar is in the bloom of development in Hong Kong. Subsequent to the seminal work of Cheung Hung Nin (1972), a number of different issues have been addressed in the joint efforts of scholars and students, notably utterance-final particles (see, for example, Kwok, 1984; Luke, 1990), some detailed analyses on a particular word class (see, for example, Luke and Nancarrow, 1998; Lau, 1999), and some contrastive analyses on distinctive features between English and Cantonese grammar (see, for example, Nancarrow and Luke, 1998). Cantonese adverbs, however, have so far received relatively scant attention in the linguistic literature.

This chapter establishes at the outset how adverbs are identified in Cantonese, based on the illuminating criterion given by Zhu Dexi (1982). It provides a sketchy overview of the previous accounts about Cantonese adverbs. It also states the objectives of the present study which are primarily devoted to discussing the morphological characteristics, semantic subcategorisation and syntactic properties. It then outlines the methodology by which a list of Cantonese adverbs is obtained through various sources. Finally, it summaries how this thesis is organised.

\subsection{IDENTIFICATION OF ADVERBS}

Different linguists might have different opinions on classifying adverbs. Quirk and Greenbaum (1973:125), for example, identify the adverbs in the English language according to their morphological characteristics and syntactic functions. 
They point out that most of the adverbs are marked morphologically by a derivational suffix $-l y$ and syntactically function as either an adverbial or a modifier of adjective and adverb.

The Cantonese adverbs, unlike English ones, do not have any significantly prominent morphological markers of adverb. Hence, the adverbs in Cantonese need to be identified syntactically, that is, according to their grammatical functions. Cai Jianhua (1995a:28) clearly states that Cantonese adverbs, like their Mandarin Chinese counterparts, function as adverbial by putting before a verb, adjective or noun. They cannot independently function as predicate nor subject of the sentence.

In fact, Cai supports the view of Zhu Dexi (1982:192) that an adverb is only used as an adverbial but not any other sentence constituents like subject and predicate. ${ }^{1} \quad$ An adverb, therefore, is identified solely by its syntactic function as adverbial. An adverbial, as Zhu Dexi (1982:151-152) defines earlier, is a modifier of any predicative element which might include, generally, verbs and adjectives, and rather specially, quantifiers, "quantifier + measure word" structure and "quantifier + measure word + noun" structure so long as they function as the predicate of the sentence. $^{2}$

Detailed treatment of how adverbs are identified and distinguished from other word classes is given in the next chapter.

\subsection{Previous Analyses on AdVerbs}

Previous accounts of adverbs have been very limited in English as adverb has long been considered as a heterogeneous category being hard to be subject to a systematic analysis (Macaulay, 1995:41; Nakamura, 1997:247-248). Cantonese is no exception. Only a few of the previous studies tend to highlight the grammatical

\footnotetext{
1 "我們把副詞定義爲只能充任狀語的虛詞。"

2 "狀語是謂詞性成分的修飾語。數詞, 數量詞, 數量名結構都是體詞性的, 但同時又策有謂詞 的性質, 所以也受狀語修飾。"
} 
meanings expressed by some adverbs in Cantonese: Chen Baoru (1982); Chen Huiying (1985); Cheng Ting Au (1990); Mai Yun (1993); Matthews and Yip (1994); Cai Jianhua (1995a, 1995b, 1997, 1998); Chen Xiaoming (2001).

Chen Huiying (1985:297-304), for instance, discusses the grammatical meanings and functions of two adverbs, 噉 gam2 and 咁 gam3. Though these two adverbs have similar phonetic forms, they differ from each other grammatically.

According to Chen, the adverb ${ }^{3}$ 噉 gam2 expresses the way in which an action occurs, and thus it normally does not modify adjectives which describe a state rather than an action.

(1) 大家搏命噉練。

daai6gaal bok3meng6-gam2 lin6

we desperate-in-such-a-way practise

"We are practicing desperately."

In this example, the adverb 噉 gam 2 indicates that the people as the subject are undergoing the action named by the predicate 練 lin6 in such a way that they are doing it desperately.

On the other hand, the adverb 咁 gam3 is usually associated with adjectives to indicate a high degree of the state attained by the adjectives.

(2) 再飲一啖咁多㖭。

zoi3 jam2 jat1 daam6 gam3 do1 tim 1

again drink one mouthful so much FP

"Just drink one more mouthful."

The above example illustrates that the adverb 咁 gam3 is used to modify the adjective 多 dol "much" so as to intensify the degree of the state being "much".

\footnotetext{
${ }^{3}$ See Peng Xiaochuan (2001) for the discussion of 噉 gam2 as an adverbial particle or marker.
} 
Chen adds that this adverb is sometimes put before stative verbs and in set verb phrases to express the same grammatical meaning, as shown in the following two examples.

(3) 咪咁㭷喇!

mai5 gam3 laul laal

not so angry FP

"Don't be so angry!"

(4) 石頭喺山上轆落洯, 好似落雨咁落。 sek6tau4 hai2 saan1 soeng6 luk1 lok6lai4 hou2ci5 lok6 jyu5 gam3 lok6 stones at hill above roll down come-down similar-to fall rain so fall "The stones rolled down like rain."

In the former example, the adverb 咁 gam3 modifies the stative verb 㽖男 lau 1 whereas in the latter, the adverb 咁 gam3 appears in a set verb phrase with the "好似 hou $2 c i 5+$ Verb $_{0}$-Object + 咁 gam3 + Verb $_{0} "$ structure.

Cai Jianhua (1997:176-179) briefly discusses three major grammatical meanings of the adverb 都 doul. Extensive research has been done on the adverb 先 $\sin 1$ (see, for example, Cheng Ting Au, 1990; Cai Jianhua, 1995; Luke Kang Kwong, 2002). Cheng (1990:189-192) basically analyses the grammatical meanings expressed by the adverb 先 $\sin 1$. He points out that the adverb has two major grammatical meanings, one of which is to indicate an action happens earlier than another, and the other of which is equal to that of the Mandarin Chinese adverb 才 cái. Cai (1995b:69-72) also discusses the grammatical meanings of the adverb 先 $\sin 1$ by considering the different constructions and contexts in which the adverb occurs. Cai proposes that the adverb can occur after a verb, a verb and its object and a verb with its complement in two contexts, namely monologue and dialogue, to express the following grammatical meanings. 
(a) earlier in time

(5) 我走先复。

ngo5 zau2 $\sin 1$ laa 3

I leave first FP

"I leave first."

(b) prerequisite

(6) 我有錢先買喇。

ngo5 jau 5 cin $2 \sin 1$ maai5 laal

I have money then buy

"If I have money, I will buy (it)."

(c) doing something seriously

（7）我哋食七嘢先?

ngo5dei6 sik6 mat lje5 sin 1

we eat what after-all

"What do we eat?"

(d) putting off a matter until later

（8）唔使理咁多，拎過洯先。

m4sai2 lei5 gam3 do1 ling1-gwo3-lai4 sin1

not care so much bring-COMP-COMP first

"You don't need to care so much of (it). Just bring (it) over."

The first two grammatical meanings coincide with that given by Cheng. However, Cheng is aware of the existence of a sentence-final particle which has exactly the same form as the adverb 先 $\sin 1$. In fact, Mai Yun (1993:66-67) clearly points out that there is a temporal sentence-final particle 先 $\sin 1$ which expresses the other two grammatical meanings mentioned by Cai.

Apart from focusing on the grammatical meanings of certain adverbs, previous studies have on occasion been devoted to different perspectives of analysing adverb properties; for instance, Chen Baoru (1982) selected eleven adverbs in Cantonese, and studied their positioning in a clause Moreover, a recent study carried out by 
Cai Jianhua (1998) stresses the importance of discerning a word to be an adverb. Cai (1998:91-94) convincingly demonstrates that the word 有 jau5 is an adverb meaning "certainly" when it comes before a verb.

(9) 我有返工。

ngo5 jau5 faanl gungl

I certainly go work

"I have certainly gone to work."

He asserts that the word 有 jau 5 is an adverb on the grounds that its negative counterpart 無 mou5 is an adverb, and the grammatical meaning "certainly" is expressed only when it is placed in front of a verb, where an adverb normally occurs.

In short, most of the previous studies on Cantonese adverbs put their emphasis on the peculiarities of individual adverbs. However, reports on the general properties of the class of adverbs have been very limited (see Huang, 1975; Jacobson, 1978; Matthews and Yip, 1994). Hence, the present study attempts to fill this gap by giving a comprehensive analysis of the Cantonese adverbs.

\subsection{ObJeCtives of PRESENT Research}

In the preceding sections, I have reviewed a number of previous accounts in the study of adverbs and found that adverbs, drastically divergent from other lexical categories, form a heterogeneous class that precludes systematic treatment. This makes linguistic literature on adverbs extremely rare and highly restricted to a few problematic adverbs and, merely, to the semantic aspect of these adverbs. It is hardly sufficient to just scratch the surface of such a complex class as adverbs. Clearly, there is much more work to be done on, particularly, morphological and syntactic characteristics of adverbs. In this respect, the present research attempts to achieve the following objectives: 
(a) find out all the Cantonese adverbs including both the Cantonese adverbs equivalent to their Mandarin Chinese counterparts and those adverbs which are specific to Cantonese only;

(b) investigate the morphological form of Cantonese adverbs;

(c) classify the Cantonese adverbs into subgroups on the basis of their grammatical meanings, being justified in terms of syntactic characteristics;

(d) discuss the syntactic functions they fulfil.

\subsection{METHODOLOGICAL ISSUES}

Cantonese adverbs in this study have been discerned by a variety of criteria. This processing will be reviewed briefly in this section. Following on from this, how the data was collected in this study will be presented (section 1.4.2) and the way the data to be represented described (section 1.4.3).

\subsubsection{Identification Criteria}

As the present study aims to give a list of Cantonese adverbs and discuss the grammatical aspects of these adverbs, the identification of adverbs is crucial. There seems to be a controversy in identifying an adverb in Mandarin Chinese, as shown in considerable discrepancies among different dictionaries on their compiled list of adverbs. On the one hand, dictionaries vary in their criteria of identification and sometimes include those adjectives and nouns that are not adverbs at all. On the other hand, dictionaries may miss out the right candidates of adverbs converted from verbs. In both cases, dictionaries do not agree with one another.

In the present study of the Cantonese adverbs, my strategy is to follow Zhu Dexi's analysis (1982:192) i.e. to identify those words which can function merely as adverbial but not any other sentence constituents like subject, object, predicate and complement as adverbs. 
On the basis of the adverbs in Mandarin Chinese reported in the dictionaries, adverbs in Cantonese which seem to be equivalent to adverbs in these dictionaries in both meaning ${ }^{4}$ and form, and most importantly, adhere to the criterion that they should function only as adverbial, have been identified as Cantonese adverbs having cognates in Mandarin and marked as $\mathbf{C M}$ under type in Appendix 1.

Apart from finding out those Cantonese adverbs which have their counterparts in Mandarin Chinese, adverbs which are Cantonese-specific have also been explored in the study. When an adverb in Cantonese is not equivalent to any adverb in Mandarin Chinese in terms of meaning and form, it is Cantonese-specific. These Cantonese-specific adverbs are represented by the type $\mathbf{C S}$ in Appendix 1 .

It should be noted that only the Cantonese words used in Hong Kong have been examined. Those used elsewhere in other provinces of the mainland China or in the Chinese communities of any other countries have not been taken into consideration in this thesis.

Moreover, particular attention was given to distinguishing adverbs from adjectives, time words (nouns) and conjunctions. This particular issue will be dealt with thoroughly in the next chapter.

\subsubsection{Collection of Data}

\subsubsection{Corpus}

The data under investigation are taken from the Cantonese corpus of naturally occurring conversations, compiled by Luke and Nancarrow (see Luke and Nancarrow (1997) for a description of the corpus). The corpus, totalling 200,000 words, consists of some story-telling and plenty of face-to-face, two-or multi-party

\footnotetext{
${ }^{4}$ In some cases, for example, 就 zau6, the Cantonese adverbs may have additional meanings that are lacking in the Mandarin equivalents. However, since they share nearly all the meanings of their Mandarin equivalents, they are not Cantonese-specific adverbs.
} 
conversations between interlocutors of both sexes and of different age groups and social classes. As a result, the database is fairly representative of the speech of people in Hong Kong and not skewed towards the usage of a particular group.

In the description of grammatical phenomena, corpus-based findings can make important contributions (Tao Hongyin, 2000:68-69). Zhan Bohui (2001:3) insightfully notes that in the study of the grammatical system of a dialect, a corpus which features widely known stories narrated by native speakers and everyday conversations among native speakers would be vital as only this sort of natural language texts would precisely reflect the real picture of different intertwined grammatical phenomena. Having met these two important features of a corpus mentioned by Zhan, the corpus has been used to collect data on the possible adverbs specific to Cantonese, example sentences of certain adverbs, and more importantly, on the discussion of syntactic functions of Cantonese adverbs.

\subsubsection{Dictionaries}

The following dictionaries, both Mandarin Chinese and Cantonese ones, have been consulted.

Mandarin Chinese dictionaries/reference books:

Hou, Xuechao. 1998. Xiandai Hanyu xuci cidian [A Dictionary of Modern Chinese

Function Words]. Beijing: Peking University Press.

Jiang, Huichuan, Xu, Haoguang, Liu, Yanxin, \& Song, Fengying. 1989. Xiandai

Hanyu fuci fenlei shiyong cidian [A Practical Classified Dictionary of Modern

Chinese Adverbs]. Beijing: Duiwai Maoyi Jiaoyu Chubanshe.

Lü, Shuxiang. 1999. Xiandai Hanyu babai ci: zengdingban [Eight Hundred Words in

Modern Chinese (a revised and enlarged edition)]. Beijing: Commercial Press.

Qufu Shifan Daxue Benshu Bianxiezu. 1992. Xiandai Hanyu Changyong xuci cidian

[A Dictionary of Common Function Words in Modern Chinese]. Hangzhou:

Zhejiang Jiaoyu Chubanshe.

Wang, Hai. 1992. Hanying xuci cidian [A Chinese-English Dictionary of Function

Words]. Beijing: Huayu Jiaoxue Chubanshe.

Wang, Ziqiang. 1984. Xiandai xuci yongfa xiao cidian [A Mini-dictionary of Modern 
Chinese Function Words]. Shanghai: Shanghai Cishu Chubanshe.

Cantonese dictionaries/reference books:

Li, Xinkui, Huang, Jiajiao, Shi, Qisheng, Mai, Yun, \& Chen, Dingfang. 1995. Guangzhou fangyan yanjiu [Research in Yue Dialects]. Guangdong: Guangdong Renmin Chubanshe.

Mai, Yun, \& Tan, Buyun. 1997. Shiyong Guangzhouhua fenlei cidian [A Practical Classified Dictionary of Cantonese] Guangdong: Guangdong Renmin Chubanshe.

Qiao, Yannong. 1966. Guangzhouhua Kouyuci de yanjiu [A Study of Colloquial Cantonese]. Hong Kong: Huaqiao Yuwen Chubanshe.

Rao, Bingcai, Ouyang, Xueya, \& Zhou, Wuji. 1996. Guangzhouhua fangyan cidian [A Dictionary of Dialectal Words in Yue Dialects]. Hong Kong: Commercial Press.

Rao, Bingcai, Ouyang, Xueya, \& Zhou, Wuji. 1997. Guangzhouhua cidian [A Dictionary of Yue Dialects]. Guangdong: Guangdong Renmin Chubanshe.

Wu, Kaibun. 1997. Xiangganghua cidian [Hong Kong Cantonese Dictionary]. Guangdong: Huacheng Chubanshe.

Zeng, Zifan. 1999. Guangzhouhua Putonghua kouyuci duiyi shouce (zengdingban) [Colloquial Cantonese and Putonghua Equivalents (a revised and enlarged edition)]. Hong Kong: Joint Publishing.

Zhang, Liyan, \& Ni, Liehuai. 1999. Gangshi Guangzhouhua cidian [A Dictionary of Hong Kong Cantonese]. Hong Kong: Wan Li Book Company.

Cheng, Ting Au. 1997. Xianggang Yueyu cidian [A Dictionary of Hong Kong Yue Dialects]. Nanjing: Jiangsu Jiaoyu Chubanshe.

\subsubsection{Mass Media}

Some example sentences regarding the actual usage of a particular adverb is adapted from the utterances heard in some local radio phone-in programs $s^{5}$ and Cantonese-speaking films.

\footnotetext{
${ }^{5}$ For example, Commerical Radio's 《芝 See 菇 Bi Family》 [Chi's Family] and 《豁達崇拜》 [Enlightened Pilgrimage] broadcast on Monday to Friday from 12:00 a.m. to 2:00 a.m. and on Sunday from 1:00 p.m. to 7:00 p.m. , respectively, and Radio Television Hong Kong's 《星空奇遇鐵達尼》 [Adventure on Titanic] broadcast on Mon to Fri from 9:00 p.m. to 12:00 p.m.
} 


\subsubsection{Native-speaker Introspection}

Some dictionaries as mentioned above clearly state the word class of certain words. When the information of a word given by the dictionaries is in conflict with the authentic data, native intuition prevails. The data under investigation have been considered by a number of native speakers until a final verdict is accepted by a majority of them.

\subsubsection{Presentation of Data}

\subsubsection{Use of Parentheses and Slash}

The parentheses "( )" represent that the Cantonese character bracketed is optional. The adverb in which the character occurs can still retain the same meaning without it. For example, the adverb 啱(啱)先 ngaam1(ngaam1) sin1 has two variants in form, both 啱先 ngaam $1 \sin 1$ and 啱啱先 ngaam 1 gaam $1 \sin 1$. These two forms of the same adverb share the same meaning "just".

The use of a slash " / " indicates that two synonymous adverbs differ only in one character. For instance, one of the entries, 唔淨只/唔單只 m4zing6zi2/ $m 4 d a a n 1 z i 2$, in the findings represent two separate adverbs 唔淨只 $m 4 z i n g 6 z i 2$ and 唔單只 m4daan1zi2, both of which differ by one character in the middle. They are equivalent to each other in meaning and actual use. Similarly, the entry 啱啱好喏 啱線 ngaam1ngaam1hou2/ngaam1ngaam1sin3 represents two adverbs, 啱啱好 ngaam1ngaam1hou2 and 啱啱線 ngaam1ngaam 1 sin3, both of which mean "just right".

\subsubsection{Translation of Cantonese Examples}

The Cantonese examples normally comprise four elements. These examples start with the Cantonese characters officially published by the Government in the 
Hong Kong Supplementary Character Set ${ }^{6}$. They are followed by the romanisations which are used in the LSHK (Linguistic Society of Hong Kong) Jyutping System. This system is different from the traditional Yale system in the following aspects. The romanisations are followed by the literal gloss of each of the words in the Cantonese examples. The free translations based on English are given finally.

$\begin{array}{lll} & \text { Yale system } & \text { LSHK Jyutping system } \\ \text { Initial consonant } & \mathrm{j} & \mathrm{z} \\ & \mathrm{ch} & \mathrm{c} \\ \text { Vowel } & \mathrm{y} & \mathrm{j} \\ & \mathrm{eu} & \text { oe (long); eo (short) } \\ \text { Tone } & \mathrm{a} & \text { aa } \\ & \text { distinguish high-level } & \text { not distinguish } \\ & \text { and high-falling tones } & \\ & \text { letters and diacritics } & \text { numbers 1-6 }\end{array}$

Note: The differences between the Yale system of romanisation and the LSHK Jyutping system are mentioned in one of the publications by the Linguistic Society of Hong Kong (1997:xviii).

\subsubsection{Final Remarks}

The Cantonese adverbs are typically found in a number of ways in order to reflect the actual usage of each adverb in colloquial Cantonese and to reduce the idiosyncrasies of native speakers in the use of a particular adverb.

\subsection{ORGANISATION OF THESIS}

Chapter One, this chapter, gives a review of the previous studies on Cantonese adverbs, establishes the research area and describes the research methodology adopted in the present study, including the way the data under investigation were collected and presented. Chapter Two discusses how to distinguish adverbs from other word classes, namely time words (nouns), adjectives and conjunctions. Chapter Three and subsequent chapters deal with the general properties of the

\footnotetext{
${ }^{6}$ It can be download from the web page http://www.info.gov.hk/digital21/chl/hkscs/download.html .
} 
Cantonese adverbs set out in (b) to (d) of the objectives. Chapter Six is the conclusion which suggests possible areas for further research and acknowledges the implications of the present study, followed by appendices and references. 


\section{Other Word Classes}

As we will be concerned with the category adverb and its various aspects, it is best to establish in advance of any discussion some agreement as to what is an indisputable, uncontroversial member of that category.

This chapter presents previous literature on the discussion of adverbs as a word class. Since Cantonese is one of the dialects of Chinese, it must share, partially, with Chinese, in both vocabulary and grammar system. In particular, adverbs in Cantonese should include some of that of Chinese. Hence, the research on adverbs in Chinese must serve as a good reference and will be discussed below.

\subsection{TOWARDS A DEFINITION OF ADVERBS}

Different grammarians have given different criteria for classifying adverbs. Wang Li (1959:21) states that adverbs are the words that express the meanings of degree, scope, time, possibility, negation, etc. and cannot be used independently to refer to entities, attributes and facts. In other words, he makes use of the grammatical meanings expressed by adverbs as criteria to classify them. Ding Shengshu et al. (1979:7) classify adverbs by their functions in a phrase. He points out that adverbs modify main verbs, auxiliary verbs, prepositions, adjectives and other adverbs.

The classification based on meanings like the one mentioned by Wang Li should be avoided lest it might be subjective, as suggested by Lü Shuxiang (1979:42) and Li 
Quan (1996:366). The criteria based on functions of a phrase by Ding Shengshu are rather limited in that adverbs could function in a clause. A better, to my knowledge, the best, criterion to classify adverbs is given by Zhu Dexi (1985:20). He defines adverbs as words which merely function as adverbials but not any other elements in a clause such as predicate and complement. ${ }^{7}$ Li Quan (1996:376) confirms Zhu's analysis by arguing that though some adverbs appear to function as complement in a clause, they are exceptions: only 很 hěn "very" and 極 $j 1^{\prime \prime}$ "very much" are used in this particular function. Citing Lu Jianming and Ma Zhen (1999:175), Li also convincingly argues that though some adverbs can be used as "predicate" in a clause, they are specially used only in speech where ellipsis is a commonplace, and they thus should not be treated as predicate because the real predicate can be recoverable from the context.

Zhu's criterion of classifying adverbs can be tested by using a pair of words in Cantonese, 頭先 tau4sin1 and 啱啱 ngaam1ngaam1.

(a) classifying by meaning: it is impossible because they are the same in meaning "just now";

(b) classifying by function: the above criterion of classifying adverbs as being only adverbials can be applied here. 啱啱 ngaam Ingaaml is an adverb because it can only function as adverbial but not other sentence constituent. 頭先 $\operatorname{tau} 4 \sin 1$ is not an adverb because it can function as object of preposition in 到頭先 dou3tau4sinl "until just now". It is a time word.

Lu Jianming (1999:406-409) provides a systematic framework in tackling classification of words in which words are properly classified according to different conditions. Each condition is considered as one level in this hierarchical framework as shown in the following figure (see next page).

7 "比較合理的方法是把副詞定義舀只能做狀語的詞。'只能做狀語'這句話蘊含著兩項語法特徵。
一是能做狀語, 二是不能做其他句法成份。" 
Figure 1: A framework of classifying adverbs

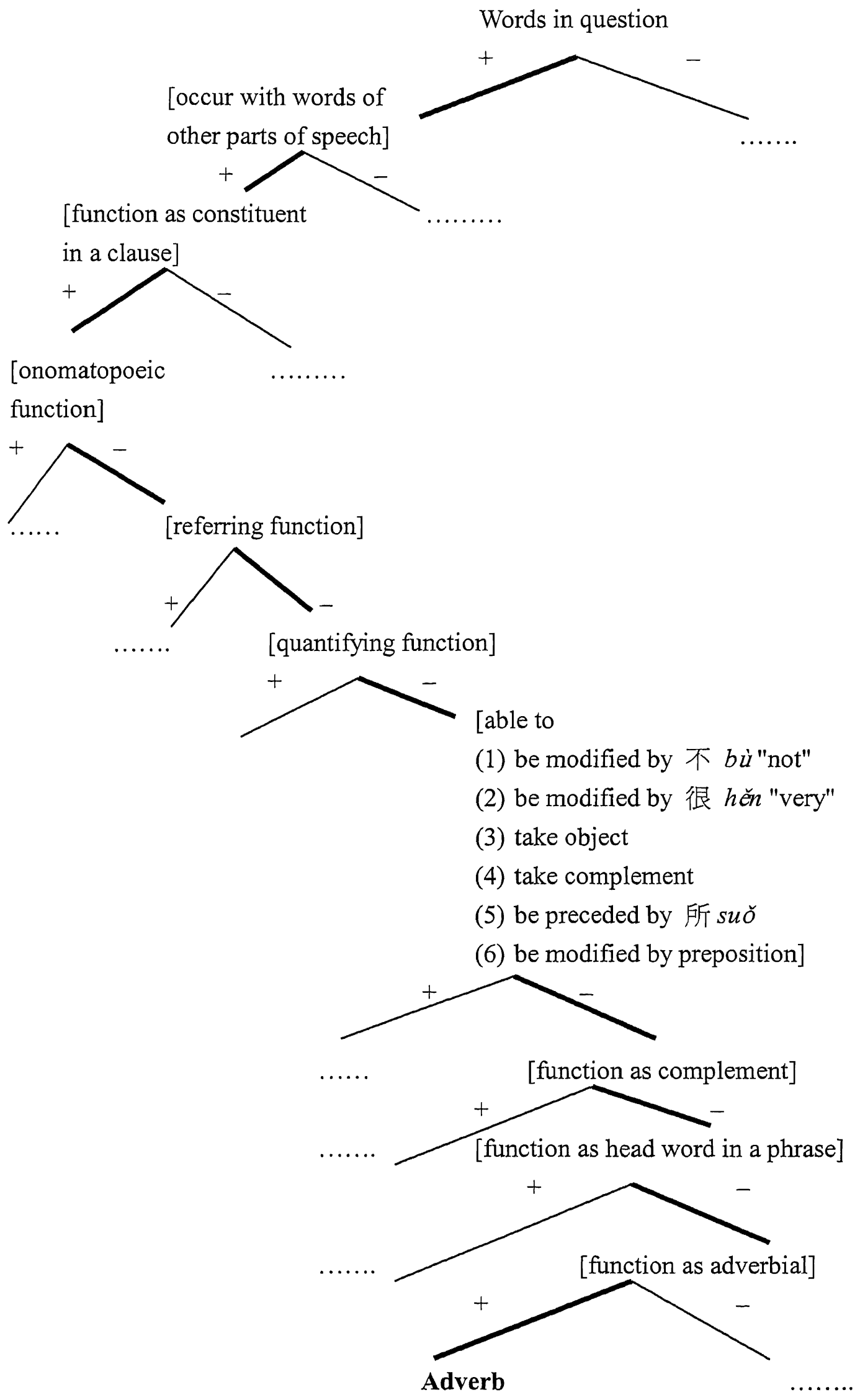


In Figure (1), a simplified version of Lu's framework, each condition is put inside square brackets. If a word satisfies the condition, it follows the route marked by "+". If not, it follows the route marked by "-". The above figure shows all the conditions fulfilled by adverbs that they do not perform any function other than adverbial. Lu therefore echoes the view proposed by Zhu Dexi on the classification of adverbs.

Before examining the various ways in which adverbs are distinguished from other word classes, a basic distinction needs to be drawn between the terms adverb and adverbial, since both are relevant to the present study. Hoye gives the best perspective in which these two terms can be understood to be separate entities.

Syntactically, adverbs can be related to such questions as Where?, When?, How long?, How often?, Why?, How? and, once this is done, the functional equivalence of multi-word units immediately becomes apparent. Thus, the question 'When will you leave?' may elicit any one of the following responses: 'Tomorrow' (adverb of time), 'Very soon' (adverb phrase with 'soon' as its head), 'The day after tomorrow' (noun phrase), 'In a couple of hours' (prepositional phrase), 'When I see fit' (finite clause). On the basis of functional analogy with the corresponding part of speech, namely adverbs, these diverse realizations are commonly referred to as adverbials. 'Adverb' is thus a word-class whereas 'adverbial' is a syntactic unit which contrasts with the other elements of clause structure, subject, verb, object and complement.

(Hoye, 1997:140-141; emphasis mine)

It is the single-word, adverb, with realisation of the adverbial function which is the primary focus in this study. One final terminological issue: the term adverb phrase above refers to a non-clausal structure consisting of two or more words with an adverb as its head: "quite [premodifier] possibly [head]".

To conclude, adverbs should be classified as words that function only as adverbials and never as the other element/constituent of sentence structure. I shall use this as a touchstone of what an adverb is. 


\subsection{As Distinguished FROM ADJECTIVES, NOUNS (TIME WORDS) AND SUBORDINATE CONJUNCTIONS}

Apart from adverbs, other word classes like adjectives and nouns (time words) can function as adverbials as well. On the other hand, adverbs can sometimes function like subordinate conjunctions to link a subordinate clause with a main clause as in the sentence 佢去, 我就去。keoi5 heoi3 ngo5 zau6 heoi3 "If he comes, I will come." It is therefore necessary to distinguish adverbs from these word classes.

\subsubsection{Adverbs and Adjectives}

Zhu Dexi (1982:192) suggests that besides functioning as adverbials, adjectives can function as modifier, predicate and complement but adverbs can function only as adverbial in a clause. In Cantonese, for instance, 好彩 hou2coi2 "lucky", 求其 kau4kei4 "perfunctory" and 無謂 mou4wai6 "pointless" are adjectives but not adverbs, as they can function as predicate, complement and modifier respectively.

(1) 今次真係好箖。(predicate) gaml ci3 zan1hai6 hou2coi2 this time really lucky "It's really lucky this time."

(2) 佢做嘢做得好求其。(complement) keoi5 zou6 je5 zou6-dakl hou 2 kau4kei4 $\mathrm{s} /$ he work thing work-COMP very perfunctory "He works very perfunctorily."

（3）佢問埋晒啲無謂嘅問題。(modifier) keoi5 man6-maai4-saai4 dil mou4wai6 ge3 man6tai4 s/he ask-COMP-COMP PL pointless GE question

"All he has asked are meaningless questions." 


\subsubsection{Adverbs and Nouns (Time Words)}

Chao Yuen Ren (1968:165) states that "a distinction should be made between (1) adverbs of time, and (2) time words." He suggests one way to distinguish time words from time adverbs that time words can serve as object of the preposition 到 dào "until" but time adverbs cannot. He demonstrates that it is possible to say 到現 在 dào xiàn zài "until present" but not *到就 dào jiù "until soon"; therefore 現在 is a time word and 就 is a time adverb. In Cantonese, for example, 最近 zeoi3gan6 "recently" and 收尾 saulmeil "at last" are time words but not time adverbs because they can be object of the preposition 到 dou3 "until".

A more comprehensive analysis on the differences between time words and time adverbs is provided by Chao Enfong (1998). She identifies two major differences:

(a) Though both time words and time adverbs can function as adverbial, only time words can function as other sentence constituents such as subject, modifier, object, etc.

(b) Time words can be preceded by prepositions but time adverbs cannot. This second major difference is similar to Chao Yuen Ren's test of using 到 dào, which is a preposition.

To sum up, time words can be a sentence constituent like subject and modifier besides functioning as adverbial. They can also be object of preposition. They are thus syntactically distinguished from adverbs which cannot be other sentence constituents except adverbial.

\subsubsection{Adverbs and Conjunctions}

Previous studies of Chinese grammar have distinguished adverbs from conjunctions. Chao Yuen Ren (1968:791) identifies that "if a word marking the relations of clauses and sentences cannot follow but must precede a subject, then it 
must be treated as a conjunction". However, as noted by Chao earlier on, a conjunction can follow the subject if both the subordinate and main clause share the same subject (113-114); if the subjects of these two clauses differ, the conjunction should be put before the subject of the clause in which it occurs. Lü Shuxiang (1979:45) suggests that words which can occur before and after the subject of a clause should be conjunctions whereas words which cannot come before the subject and only after it should be classified as adverbs.

At first glance, Chao's views on the positioning of a conjunction in a clause seems not very consistent as on the one hand, a conjunction should come before the subject, but it can occur either before or after the same subject shared by the main and subordinate clause on the other. However, as Zhang Baolin (1996) comments, conjunctions can be divided into two types according to Chao's analysis. One group occurs only before the subject and the other can occur before or after the subject. Concerning the distribution, these two types of conjunction are common in one of the positions of occurrence, that is, before the subject. We can come to a conclusion that the word which occurs before the subject must be a conjunction whereas the one which occurs only after the subject must be an adverb.

Though the above means to differentiate between adverbs and conjunctions seems reasonable, it can be proven wrong rather easily as some adverbs, for example, 可能 ho2nang4 "perhaps" can take a sentence-initial position, i.e. before the subject. In view of this problem, Zhang (1996:399) concludes that both the position and grammatical meaning of a word should be taken into account in determining whether it is an adverb or a conjunction.

(a) If a word serves to link two clauses and occurs only before the subject, it must be a conjunction.

(b) If a word serves to link two clauses and occurs either before the subject or after the subject, it must be a conjunction.

(c) If a word serves to link two clauses and occurs only after the subject, it must be an adverb. 
(d) If a word does not link two clauses, it might be an adverb or of any word class other than adverb and conjunction.

My own position is no different from these previous accounts. I believe that conjunctive adverbs i.e. adverbs that serve a linking function can only come after the subject in Cantonese. Conjunctions, however, can come before or after the subject. Hence in Cantonese 一係jat1hai6 "alternatively" and 事關 si6gwaan1 "because" are conjunctions, not adverbs at all, as they can come before the subject.

(4) 你而家唔得閒, 一係我轉頭再啾過。 nei5 ji4gaal $m 4$ daak1 haan4 jat 1 hai6 ngo5 zyun3tau4 zoi3 lai4-gwo3 you now not available alternatively I later again come-ASP "You are not available now. Maybe I come again later."

(5) 佢唔啾得, 事關佢今日約咗人。 keoi5 m4 lai4-dak1 si6gwaan1 keoi5 gamljat6 joek3-zo3 jan4 s/he not come-COMP because s/he today has-ASP-an-appointment people "She couldn't come because she has an appointment with someone."

\subsection{SUMMARY}

Words which can only function as adverbial but not any other sentence constituents such as subject, object, complement, etc. are classified as adverbs. This criterion helps distinguish adverbs from other word classes, particularly adjectives and nouns. It is difficult to distinguish adverbs from conjunctions as both perform a linking function between two clauses. In such a case, the positioning of occurrence plays a determinant role in that adverbs can occur only after the subject in linking up two clauses. 


\section{of Adverbs \\ Forms of Adverbs}

This chapter will mainly focus on five aspects concerning the form of Cantonese adverbs. These five aspects are: (1) simple and complex adverbs, (2) patternings shared by various adverbs, (3) reduplicated constructions, (4) pairs of adverbs in coordination and (5) pairs of adverbs in antonymy.

\subsection{SimPle AND COMPLEX ADVERBS}

Adverbs fall into two major classes on morphological grounds. In this study, most of the monosyllabic and disyllabic adverbs are morphologically simple in the sense that they do not have any internal structure. By contrast, trisyllabic and polysyllabic adverbs are morphologically complex in that their internal structure encompasses, for example, different forms of reduplication patterns or coordination.

More than half of the Cantonese adverbs are disyllabic, constituting 63\% $(343 / 545)$ of the total number of adverbs found in the present study. In particular, nearly most of the Cantonese adverbs which are equivalent to their Mandarin Chinese counterparts are disyllabic. Of the 51 monosyllabic adverbs, about half are specific to Cantonese and about half have equivalents in Mandarin Chinese. In contrast, trisyllabic adverbs (totalling 99 instances) and polysyllabic adverbs (totalling 52 instances) seem to be more prominent in Cantonese-specific adverbs than in adverbs equivalent to those in Mandarin Chinese. This preponderance of trisyllabic and polysyllabic adverbs suggests that polysyllabicity is one of the characteristics of Cantonese-specific adverbs, as opposed to the fact that Cantonese 
adverbs equivalent to their Mandarin Chinese counterparts are typically disyllabic.

\subsubsection{Simple Adverbs}

\subsubsection{Monosyllabic Adverbs}

\begin{tabular}{|c|c|c|c|}
\hline 白 & 牛 & 初 & 重 \\
\hline baak6 & bun3 & col & cung 4 \\
\hline "in vain; for nothing" & "half" & "at first" & "again; once more" \\
\hline 都 & 乾 & 一 & 又 \\
\hline doul & gonl & jatl & jau6 \\
\hline "all; emphasis" & "in a dry manner" & "as soon as" & "repeatedly; also" \\
\hline 力 & 誤 & 漫 & 末 \\
\hline lik6 & $m 6$ & maan6 & mei6 \\
\hline "make every effort" & "mistakenly" & "casually" & "not yet" \\
\hline 無 & 每 & 偏 & 新 \\
\hline mou 5 & mui5 & $\operatorname{pin} 1$ & $\operatorname{san} 1$ \\
\hline "have not" & "whenever" & $\begin{array}{l}\text { "contrary to speaker's } \\
\text { will" }\end{array}$ & "newly" \\
\hline 死 & 算 & 偷 & 互 \\
\hline sei2 & syun3 & taul & wu6 \\
\hline $\begin{array}{l}\text { "desperately; } \\
\text { determinedly" }\end{array}$ & "fairly" & "secretly" & "each other" \\
\hline 就 & 最 & 盡 & 至 \\
\hline zau6 & zeoi3 & zeon6 & $z i 3$ \\
\hline "very soon; then" & "the most" & "exhaustively" & "then; until" \\
\hline 直 & 再 & 專 & \\
\hline$z i k 6$ & $z o i 3$ & zyun1 & \\
\hline "directly" & "again; more" & "specially; always" & \\
\hline
\end{tabular}

(The above are those Cantonese adverbs having more or the less the same counterparts in Mandarin Chinese. ${ }^{8}$ Adverbs which are specific to Cantonese are given as follows.)

${ }^{8}$ These Cantonese adverbs are checked against the Chinese dictionaries. If they happen to have the same meaning and form as the Mandarin ones, they are considered in this study to have counterparts in Mandarin. See Chapter one, section 1.4.1. 


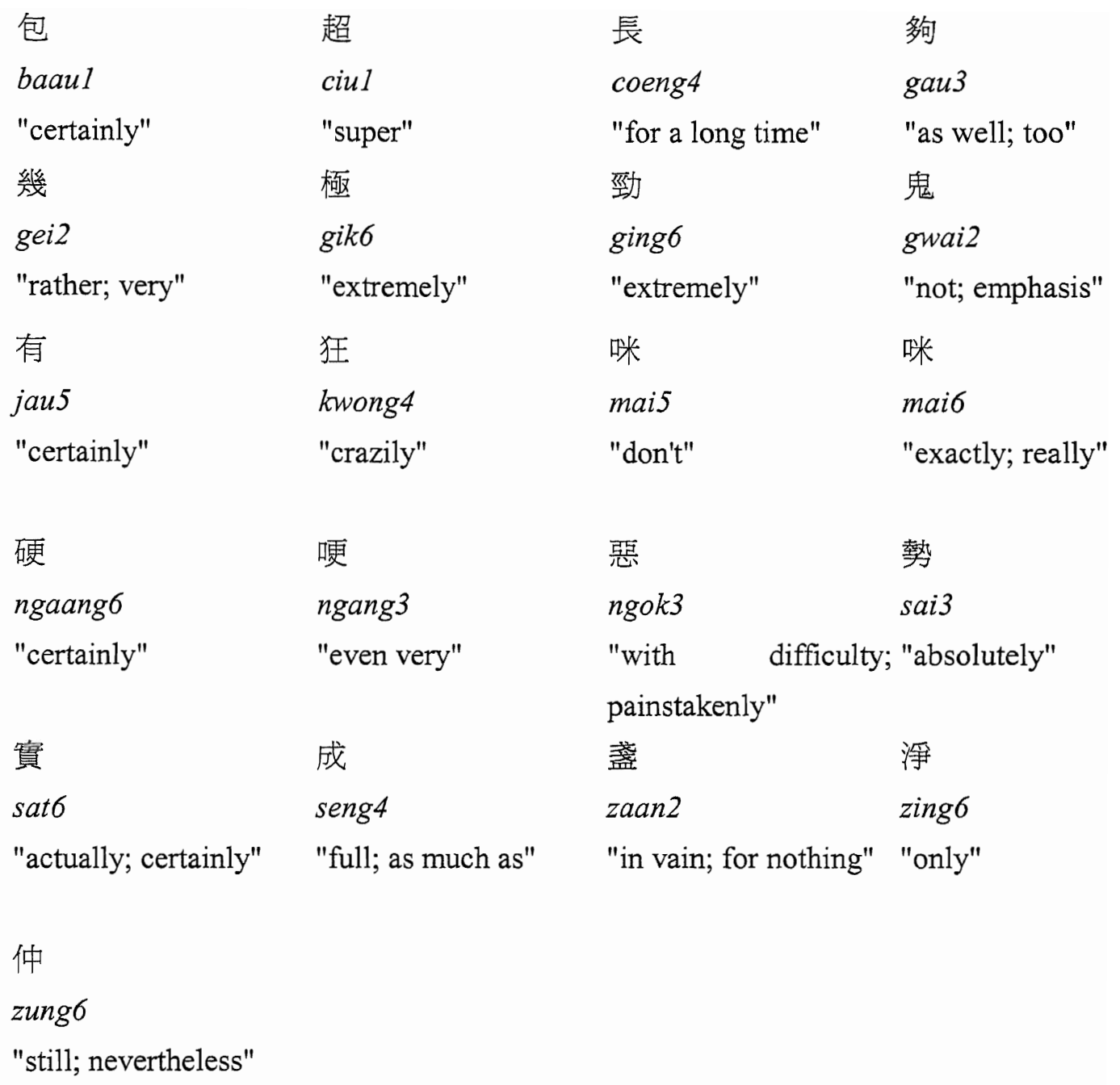

\subsubsection{Disyllabic Adverbs}

$\begin{array}{lll}\text { 百般 } & \text { baak3bun1 } & \text { "by every means" } \\ \text { 不斷 } & \text { bat1dyun6 } & \text { "continuously" } \\ \text { 不妨 } & \text { batlfong4 } & \text { "there is no harm in; might as well" } \\ \text { 不如 } & \text { batljyu4 } & \text { "it would be better to" } \\ \text { 不愧 } & \text { bat1kwai5 } & \text { " be worthy of" } \\ \text { 本來 } & \text { bun2loi4 } & \text { "originally" } \\ \text { 親自 } & \text { canlzi6 } & \text { "in person" } \\ \text { 趁早 } & \text { can3zou2 } & \text { "as early as possible" } \\ \text { 曾經 } & \text { cang4ging1 } & \text { "ever" } \\ \text { 隨處 } & \text { ceoi4cyu3 } & \text { "everywhere" } \\ \text { 隨地 } & \text { ceoi4dei6 } & \text { "anywhere" } \\ \text { 隨口 } & \text { ceoi4hau2 } & \text { "(speak) thoughtlessly" }\end{array}$




\begin{tabular}{|c|c|c|}
\hline 隨手 & ceoi4sau2 & "conveniently" \\
\hline 隨時 & ceoi4si4 & "at all times" \\
\hline 始終 & ci2zungl & "after all" \\
\hline 似乎 & ci5fu4 & "seemingly" \\
\hline 情願 & cing4jyun2 & "would rather" \\
\hline 從來 & cung4loi4 & "always; at all times" \\
\hline 重新 & cung4san 1 & "again; anew" \\
\hline 從頭 & cung4tau4 & "anew; once again" \\
\hline 從中 & cung4zungl & "from among" \\
\hline 大約 & daai6joek3 & "approximately" \\
\hline 大力 & daai6lik6 & "vigorously" \\
\hline 大略 & daai6loek2 & "roughly" \\
\hline 單獨 & daanlduk6 & "alone" \\
\hline 特別 & dak6bit6 & "particularly" \\
\hline 頂多 & ding $2 d o 1$ & "at the most" \\
\hline 當初 & donglcol & "originally" \\
\hline 當然 & dongljin4 & "of course" \\
\hline 當面 & dong1min 2 & "in somebody's presence" \\
\hline 當罡 & donglzung3 & "in the presence of all" \\
\hline 到處 & dou 3 cyu3 & "everywhere" \\
\hline 到底 & dou3dai2 & "actually; exactly" \\
\hline 反複 & faan $2 f u k 1$ & "repeatedly" \\
\hline 反而 & faan $2 j i 4$ & "on the contrary" \\
\hline 反爲 & faan2wai4 & "on the contrary; instead" \\
\hline 反正 & faan2zing3 & "anyway" \\
\hline 分明 & fanlming 4 & "clearly; plainly; evidently" \\
\hline 分頭 & fanltau4 & "separately" \\
\hline 分外 & fan6ngoi6 & "particularly" \\
\hline 簡直 & gaan2zik6 & "simply" \\
\hline 較爲 & gaau3wai4 & "relatively" \\
\hline 跟手 & gan1sau2 & "next; then; after that" \\
\hline 近乎 & gan $6 f u 4$ & "close to" \\
\hline 更加 & gang3gaal & "even more" \\
\hline 究竟 & gau3ging2 & "actually; exactly" \\
\hline 幾乎 & geilfu4 & "almost" \\
\hline 居然 & geoiljin4 & "unexpectedly" \\
\hline 極力 & gik6lik6 & "do one's utmost" \\
\hline 竟然 & ging2jin4 & "unexpectedly" \\
\hline
\end{tabular}




\begin{tabular}{|c|c|c|}
\hline 姑且 & gulce2 & "for the time being" \\
\hline 公然 & gungljin4 & "openly" \\
\hline 果然 & gwo2jin4 & "accordingly" \\
\hline 過分 & gwo 3 fan 6 & "excessively" \\
\hline 過後 & gwo3hau6 & "afterwards; later" \\
\hline 刻意 & haklji3 & "painstakingly" \\
\hline 肯定 & hang2ding6 & "surely" \\
\hline 起初 & hei2col & "originally" \\
\hline 起碼 & hei2maa5 & "at least" \\
\hline 輕易 & hinglji6 & "rashly" \\
\hline 可能 & ho2nang4 & "probably" \\
\hline 何必 & ho4bit1 & "there is no need" \\
\hline 何苦 & ho4fu2 & "why bother" \\
\hline 向來 & hoeng3loi4 & "always" \\
\hline 好似 & hou $2 c i 5$ & "presumably" \\
\hline 好在 & hou2zoi6 & "fortunately" \\
\hline \multirow[t]{3}{*}{ 一齋 } & jatlcai4 & "together" \\
\hline & & "along with all the others" \\
\hline & & "at a time" \\
\hline 一旦 & jat1 daan6 & "once; in case" \\
\hline 一定 & jat1ding 6 & "certainly; surely" \\
\hline 一度 & jat1dou6 & "for a time" \\
\hline 一共 & jatlgung6 & "altogether" \\
\hline$一 \square$ & jat1hau2 & "with certainty" \\
\hline 一向 & jat1hoeng3 & "consistently; all along" \\
\hline 一概 & jat1koi3 & "without exception; totally" \\
\hline 一律 & jat1leot6 & "without exception" \\
\hline 一連 & jat1lin4 & "in succession" \\
\hline \multirow[t]{2}{*}{ 一路 } & jatllou6 & "continuously; always; all along; all the way" \\
\hline & & "at the same time; simultaneously" \\
\hline 一味 & jat1mei2 & "blindly; endlessly" \\
\hline 一眼 & jatIngaan5 & "in a glimpse" \\
\hline \multirow[t]{2}{*}{ 一心 } & jat1saml & "wholeheartedly" \\
\hline & & "intentionally" \\
\hline 一 & jat1sau2 & "single-handed" \\
\hline 一直 & jatlzik6 & "continuously" \\
\hline 一再 & jat1zoi3 & "time and again" \\
\hline 有時 & jau5si4 & "sometimes" \\
\hline
\end{tabular}




\begin{tabular}{|c|c|c|}
\hline 依稀 & jilheil & "vaguely" \\
\hline 依然 & jiljin4 & "still" \\
\hline 已經 & ji5gingl & "already" \\
\hline 異常 & ji6soeng4 & "extremely" \\
\hline 亦(都) & jik6(doul) & "as well" \\
\hline 仍然 & jng4jin4 & "still; yet" \\
\hline 約莫 & joek3mok2 & "approximately" \\
\hline 預先 & jyu6sin 1 & "in advance; beforehand" \\
\hline 完全 & jyun4cyun 4 & "completely" \\
\hline 原來 & jyun4loi4 & "orıginally" \\
\hline 原先 & jyun $4 \sin 1$ & "orıginally" \\
\hline 及早 & kap6zou2 & "as soon as possible" \\
\hline 其實 & kei4sat6 & "actually; in fact" \\
\hline 確實 & kok3sat6 & "really; indeed" \\
\hline 立即 & laap6zik1 & "immediately" \\
\hline 連續 & $\operatorname{lin} 4 z u k 6$ & "successively" \\
\hline 另外 & ling6ngoi6 & "in addition" \\
\hline 略爲 & loek6wai4 & "slightly" \\
\hline 陸續 & luk6zuk6 & "in succession" \\
\hline 亂(咁) & lyun2(gam3) & "thoughtlessly" \\
\hline 貿然 & mau6jin4 & "rashly; hastily" \\
\hline 未必 & mei6bit1 & "not necessarily" \\
\hline 未曾 & mei6cang4 & "never" \\
\hline 未免 & $\operatorname{mei6min} 5$ & "a bit too" \\
\hline 未嘗 & mei6soeng4 & "not (negative sentence)" \\
\hline 無從 & mou4cung 4 & "have no way of doing something" \\
\hline 無非 & mou4feil & "nothing but; simply; no more than; only" \\
\hline 無日 & mou5jat6 & "all the time (negative sentence)" \\
\hline 難怪 & naan4gwaai3 & "no wonder" \\
\hline 毅然 & ngai6jin4 & "determinedly" \\
\hline 寧願 & ning4jyun2 & "would rather" \\
\hline 憑空 & pang4hung1 & "without foundation" \\
\hline 稍爲 & saau2wai4 & "slightly" \\
\hline 誓死 & sai6sei2 & "firmly" \\
\hline 新近 & san1gan 6 & "recently" \\
\hline 實則 & sat6zakl & "actually" \\
\hline 實在 & sat6zoi6 & "actually" \\
\hline 首先 & $\operatorname{sau} 2 \sin 1$ & "first of all" \\
\hline
\end{tabular}




\begin{tabular}{|c|c|c|}
\hline 純粹 & seon 4 seoi 5 & "simply" \\
\hline 順便 & seon6bin2 & "conveniently" \\
\hline 順帶 & seon6daai3 & "in passing" \\
\hline 順勢 & seon6sai3 & "by taking advantage of an opportunity" \\
\hline 順手 & seon6sau2 & "conveniently" \\
\hline 私自 & silzi6 & "secretly" \\
\hline 先(至) & $\sin 1(z i 3)$ & "first; then" \\
\hline 擅自 & $\sin 6 z i 6$ & "without authorisation" \\
\hline 索性 & sok3sing 3 & "simply" \\
\hline 素來 & sou3loi4 & "always; usually" \\
\hline 太(過) & taai3(gwo3) & "too" \\
\hline 通常 & tung1soeng4 & "usually" \\
\hline 同時 & tung 4 si4 & "at the same time" \\
\hline 或者 & waak6ze2 & "perhaps; maybe" \\
\hline 唯有 & wai4jau 5 & "only; anyway" \\
\hline 永遠 & wing5jyun 5 & "forever" \\
\hline 互相 & wu6soeng1 & "mutually; each other" \\
\hline \multirow[t]{2}{*}{ 最多至多 } & zeoi3dol/zi3dol & "at the worst" \\
\hline & & "at the most" \\
\hline 最好 & zeoi3hou2 & "it is good to" \\
\hline 最少至少 & zeoi3siu2/zi3siu2 & "at least" \\
\hline 盡情 & zeon6cing4 & "as much as one likes" \\
\hline 儘快 & zeon6faai3 & "as fast as one could" \\
\hline 盡力 & zeon6lik6 & "try one's best" \\
\hline 儘量 & zeon6loeng6 & "to the full" \\
\hline 儘早 & zeon6zou2 & "as early as possible" \\
\hline 即刻 & ziklhaakl & "immediately" \\
\hline 照例 & ziu3lai6 & "as a rule" \\
\hline 照理 & ziu3lei5 & "generally" \\
\hline 照常 & ziu3soeng4 & "as usual" \\
\hline \multirow[t]{2}{*}{ 將近 } & zoenglgan6 & "nearly; almost" \\
\hline & & "very soon" \\
\hline 再三 & zoi3saaml & "over and over again" \\
\hline 早日 & zou2jat6 & "soon" \\
\hline 足以 & $z u k 1 j i 5$ & "enough" \\
\hline 逐個 & zuk6go3 & "one by one" \\
\hline 逐一 & zuk6jat1 & "one by one" \\
\hline 終於 & zungljyul & "finally" \\
\hline
\end{tabular}




$\begin{array}{lll}\text { 總共 } & \text { zung2gung6 } & \text { "altogether" } \\ \text { 總算 } & \text { zung2syun3 } & \text { "at long last; finally" } \\ & & \text { "all things considered" } \\ \text { 專程 } & \text { zyun1cing4 } & \text { "specially" } \\ \text { 專門 } & \text { zyun1mun2 } & \text { "specially; always" } \\ \text { 絕對 } & \text { zyut6deoi3 } & \text { "absolutely" }\end{array}$

(The above are those Cantonese adverbs having more or the less the same counterparts in Mandarin Chinese. Adverbs which are specific to Cantonese are given as follows.)

\begin{tabular}{|c|c|c|}
\hline 擺明 & baai2ming4 & "clearly" \\
\hline 弊在 & bai6zoi6 & "unfortunately" \\
\hline 不舅 & bat1laul & "consistently; all along" \\
\hline 不知 & batlzil & " to speaker's/hearer's surprise" \\
\hline 查實 & caa4sat6 & "in fact; actually" \\
\hline 千祈 & cinlkei4 & "for heaven's sake" \\
\hline 初頭 & coltau4 & "at the beginning" \\
\hline 打掂 & daa2dim6 & "vertically" \\
\hline 打同 & daa2dung6 & "vertically" \\
\hline 打子子 & daa2maal & "in pair" \\
\hline 打橫 & daa2waang 4 & "horizontally" \\
\hline 大早 & daai6zou2 & "some time ago" \\
\hline 單係 & daan1hai6 & "only" \\
\hline 得滯 & daklzai6 & "too" \\
\hline 特登 & dak6dangl & "for a special purpose; specially" \\
\hline 點止 & $\operatorname{dim} 2 z i 2$ & "not only" \\
\hline 頂籠 & ding2lung2 & "at the most" \\
\hline 多數 & dolsou3 & "probably; most likely" \\
\hline 當堂 & dong1tong 4 & "immediately" \\
\hline 都 & doulhai6 & "had better" \\
\hline 到時 & dou3si4 & "at that time" \\
\hline 凡親 & faan 4 can 1 & "whenever" \\
\hline 逢(親) & fung4(can1) & "whenever" \\
\hline & fung6zi2 & "as a rule" \\
\hline 監才 & gaam $3 \operatorname{cou} 1$ & "unyieldingly" \\
\hline 監生 & gaam3saang1 & "while still alive" \\
\hline
\end{tabular}




\begin{tabular}{|c|c|c|}
\hline 夾硬 & gaap3ngaang2 & "unyieldingly" \\
\hline 計正 & gai3zeng3 & "under normal circumstances; as a rule" \\
\hline 咁(鬼) & $\operatorname{gam3}(g w a i 2)$ & "very" \\
\hline 咁啱 & gam3ngaam1 & "by chance" \\
\hline 咁滯 & gam3zai6 & "almost (positive sentence)" \\
\hline 跟住 & ganlzyu6 & "afterwards; then" \\
\hline 梗(係) & gang2(hai6) & "undoubtedly; certainly" \\
\hline 急住 & gaplzyu6 & "in a hurry; in a haste" \\
\hline 九成 & gau2sing 4 & "very likely" \\
\hline 舊底 & gau6dai2 & "in the past" \\
\hline 幾大 & gei2daai2 & "no matter (what; how; etc.)" \\
\hline 幾咁/幾鬼 & gei2gam3/gei2gwai2 & "very" \\
\hline 極之 & gik6zil & "extremely" \\
\hline 經已 & ginglji5 & "already" \\
\hline 乾脆 & gonlceoi3 & "simply" \\
\hline 鬼咁 & gwai2gam3 & "very" \\
\hline 過頭 & gwo3tau4 & "very; too" \\
\hline 喺度旿老處 & hai2dou6/hai $2 s y u 3$ & "in progress of" \\
\hline 係都 & hai6doul & "with insistence" \\
\hline 係噉 & hai6gam 2 & "continuously" \\
\hline 後尾 & hau6meil & "afterwards; later" \\
\hline 起勢 & hei2sai3 & "desperately" \\
\hline 起先 & hei $2 \sin 1$ & "at the beginning" \\
\hline 何止 & ho4zi2 & "not only" \\
\hline 响度/响處 & hoeng2dou6/hoeng2syu3 & "in progress of" \\
\hline 好(鬼) & hou2(gwai2) & "extremely" \\
\hline 好E & hou2jat6 & "rarely; seldom (negative sentence)" \\
\hline 好少 & hou2siu2 & "rarely; seldom" \\
\hline 一日 & jatljat6 & "in the final analysis; at bottom" \\
\hline \multirow[t]{3}{*}{ 一於 } & jatljyul & "regardless of the consequences" \\
\hline & & "simply; just; altogether" \\
\hline & & "expressing decision" \\
\hline 一早 & jat1zou2 & "in advance" \\
\hline 由 & jau4tau4 & "from the beginning" \\
\hline \multirow[t]{2}{*}{ 有得 } & jau5dakl & "it is possible to" \\
\hline & & "it is worthwhile to" \\
\hline & jau5ji3 & "intentionally; deliberately" \\
\hline & jau6si3 & "again" \\
\hline
\end{tabular}




\begin{tabular}{|c|c|c|}
\hline 應份 & jinglfan 6 & "necessarily" \\
\hline 認真 & jing2zanl & "absolutely" \\
\hline 預早 & jуu6zou2 & "in advance; beforehand" \\
\hline 確係 & kok3hai6 & "really; indeed" \\
\hline 嗱埋 & laa2maai4 & $\begin{array}{l}\text { "easily; frequently; at every turn" } \\
\text { "generally speaking" }\end{array}$ \\
\hline 拉匀 & laailwan4 & "on average" \\
\hline 䊖日 & laam3jat6 & "every two days" \\
\hline 笠亂 & lap6lyun2 & "carelessly; casually; at random" \\
\hline 輪流 & leon4lau2 & "in turn" \\
\hline 連隨 & $\operatorname{lin} 4$ ceoi4 & "immediately" \\
\hline 連氣 & lin4hei3 & "in succession" \\
\hline 靈舍 & ling 4 se 3 & "particularly" \\
\hline 兩份 & loeng5fan2 & "together" \\
\hline 落手 & lok6sau2 & "by oneself; in person" \\
\hline 唔多 & $m 4 d o 1$ & "not very" \\
\hline 唔慌 & m4fong1 & "absolutely not" \\
\hline 唔(鬼) & $m 4$ (gwai2) & "not" \\
\hline 唔好 & m4hou2 & "don't" \\
\hline 唔使 & $m 4$ sai2 & "not necessarily" \\
\hline \multirow[t]{2}{*}{ 唔通 } & m4tung1 & "expressing rhetorical question" \\
\hline & & "can it be that; is it possible that" \\
\hline \multirow[t]{2}{*}{ 唔知 } & $m 4 z i l$ & "unknowingly" \\
\hline & & "really" \\
\hline 唔只 & $m 4 z i 2$ & "not only" \\
\hline 猛(咁) & maang5(gam3) & "desperately" \\
\hline 也滯 & matlzai6 & "not quite (negative sentence)" \\
\hline 無得 & mou 5 dakl & "it is not possible to" \\
\hline 無セ & mou 5 mat 1 & "almost not" \\
\hline 無話 & mou 5 waa6 & "never" \\
\hline 硬係 & ngaang2hai6 & "in contrary to speaker's will" \\
\hline 怕(且) & paa3(ce2) & "perhaps" \\
\hline \multirow[t]{2}{*}{ 晨早 } & $\operatorname{san} 4 z o u 2$ & "well in advance" \\
\hline & & "early in the morning" \\
\hline 實梗 & sat6gang2 & "certainly" \\
\hline 實係 & sat6hai6 & "certainly" \\
\hline & sat6hang4 & "certainly" \\
\hline 成日 & seng4jat6 & "always" \\
\hline
\end{tabular}




\begin{tabular}{|c|c|c|}
\hline 試過 & si3gwo3 & "before; formerly; in the past" \\
\hline 是必 & si6bit1 & "certainly" \\
\hline 是但 & si6daan6 & "randomly; as one pleases" \\
\hline 誠心 & $\operatorname{sing} 4 \operatorname{sam} 1$ & "intentionally" \\
\hline 睇怕 & tai2(paa3) & "apparently; seemingly" \\
\hline 同埋 & tung4maai4 & "together" \\
\hline 話名 & waa6meng2 & "nominally" \\
\hline 話晒 & waa6saai3 & "after all" \\
\hline 枕長 & zam2coeng 4 & "for a long time" \\
\hline 枕住 & zam2zyu6 & "contınuously" \\
\hline 真係 & zan1hai6 & "really" \\
\hline 就快 & zaubfaai3 & "very soon" \\
\hline 就溙 & zau6lai4 & "very soon" \\
\hline 正話 & zeng3waa6 & "just" \\
\hline 卒之 & zeotlzil & "finally" \\
\hline 只係 & zi2hai6 & "merely; only; just" \\
\hline 即管 & ziklgun2 & "feel free to; not hesitate to" \\
\hline \multirow[t]{3}{*}{ 直情 } & zik6cing4 & "straight; directly" \\
\hline & & "simply; at all" \\
\hline & & "surely" \\
\hline \multirow[t]{3}{*}{ 直頭 } & zik6tau4 & "straight; directly" \\
\hline & & "simply; at all" \\
\hline & & "surely" \\
\hline 正 $($ - $)$ & zing3(jat1) & "indeed; really" \\
\hline 正式 & zing 3 sik1 & "indeed; really" \\
\hline \multirow[t]{2}{*}{ 淨係 } & zing6hai6 & "only" \\
\hline & & "constantly" \\
\hline 接住 & zір $3 z y u 6$ & "immediately afterwards; in a minute" \\
\hline \multirow[t]{2}{*}{ 照計 } & ziu3gai3 & "according to reason; in the ordinary course \\
\hline & & of events; normally" \\
\hline 座底 & zo6dai2 & "at least" \\
\hline 再纾 & zoi3si3 & "again" \\
\hline 撞喏 & zong6ngaam1 & "by chance" \\
\hline 早知 & $z o u 2 z i 1$ & "having known this beforehand" \\
\hline 逐啲 & $z u k 6 d i 1$ & "one by one" \\
\hline 終 & zunglseoil & "anyhow; eventually; after all" \\
\hline 總 & zung2hai6 & "always; invariably" \\
\hline 仲侎 & zung6(hai6) & "still; nevertheless" \\
\hline
\end{tabular}




\subsubsection{Trisyllabic Adverbs}

$\begin{array}{lll}\text { 雴時間 } & \text { saap3si4gaan1 } & \text { "in a twinkling" } \\ \text { 甚至(無)/甚至(乎) } & \text { sam6zi3(mou4)/sam6zi3(fu4) } & \text { "even" } \\ \text { 死命(噉) } & \text { sei2meng6(gam2) } & \text { "desperately" } \\ \text { 唯獨是 } & \text { wai4duk6si6 } & \text { "only" } \\ \text { 照舊(噉) } & \text { ziu3gau6(gam2) } & \text { "as usual" } \\ \text { 照樣(噉) } & \text { ziu3joeng2(gam2) } & \text { "1n the same old way" }\end{array}$

(The above are those Cantonese adverbs having more or the less the same counterparts in Mandarin Chinese. Adverbs which are specific to Cantonese are given as follows.)

$\begin{array}{lll}\text { 不特止 } & \text { bat1dak6zi2 } & \text { "not only" } \\ \text { 第(二)日 } & \text { dai6(ji6)jat6 } & \text { "later" } \\ \text { 第(二)時 } & \text { dai6(ji6)si4 } & \text { "next time" } \\ \text { 點(不)知 } & \text { dim2(bat1)zi1 } & \text { "unexpectedly" } \\ \text { 極其量 } & \text { gik6kei4loeng6 } & \text { "at the most" } \\ \text { 而蹦呤 } & \text { ham6baang6laang6 } & \text { "all" } \\ \text { 好多時 } & \text { hou2dolsi4 } & \text { "very often" } \\ \text { 好意思 } & \text { hou2ji3si1 } & \text { "have the nerve" } \\ \text { 一口價 } & \text { jat1hau2gaa3 } & \text { "at a fixed price" } \\ \text { 一口氣 } & \text { jat1hau2hei3 } & \text { "in one breath; without a break" } \\ \text { 一輪嘴 } & \text { jat1leon2zeoi2 } & \text { "without a break" } \\ \text { 一䁪眼 } & \text { jat1zaam2ngaan5 } & \text { "in an instant" } \\ \text { 有幾何 } & \text { jau5gei2ho2 } & \text { "rarely; seldom" } \\ \text { 有陣時 } & \text { jau5zan6si2 } & \text { "sometimes" } \\ \text { 唔見得 } & \text { m4gin3dak1 } & \text { "not likely" } \\ \text { 唔淨只/唔單只 } & \text { m4zing6zi2/m4daan1zi2 } & \text { "not only" } \\ \text { 萬二分 } & \text { maan6ji6fen1 } & \text { "very much; extremely" } \\ \text { 未有耐 } & \text { mei6jau5noi1 } & \text { "not (in time or degree)" } \\ \text { 無幾何 } & \text { mou5gei2ho2 } & \text { "rarely; seldom" } \\ \text { 而唔中 } & \text { noi6m4zung1 } & \text { "once in a while" } \\ \text { 死(人)都 } & \text { sei2(jan4)dou1 } & \text { "in any case; anyway" } \\ & & \end{array}$




$\begin{array}{lll}\text { 私底下 } & \text { sildai2haa6 } & \text { "in secret; in private" } \\ \text { 少不垐 } & \text { siu2bat1min5 } & \text { "to a certain extent" } \\ \text { 聽講(話) } & \text { tenglgong2(waa6) } & \text { "be told; heard of" } \\ \text { 玩吓手 } & \text { waan2haa5sau2 } & \text { "most likely" } \\ \text { 周(不)時 } & \text { zaul(bat1)si4 } & \text { "often; constantly" } \\ \text { 只不過 } & \text { zi2batlgwo3 } & \text { "merely; only; just" } \\ \text { 至多(咪) } & \text { zi3dol(mai6) } & \text { "at the worst" } \\ \text { 自不然 } & \text { zi6batljin4 } & \text { "naturally" } \\ \text { (老)早就 } & \text { (lou5)zou2zau6 } & \text { "in advance" }\end{array}$

\subsubsection{Polysyllabic Adverbs}

\begin{tabular}{|c|c|c|}
\hline 如何 & $\operatorname{dikl(ji4ce2)kok3}$ & "indeed; really" \\
\hline
\end{tabular}

(The above are those Cantonese adverbs having more or the less the same counterparts in Mandarin Chinese. Adverbs which are specific to Cantonese are given as follows.)

$\begin{array}{lll}\text { 第一時間 } & \text { dai6jat1si4gaan1 } & \text { "immediately" } \\ \text { 突然(之)間 } & \text { dat6jin4(zi1)gaan1 } & \text { "suddenly" } \\ \text { 叠埋心水 } & \text { dip6maai4sam1seoi2 } & \text { "wholeheartedly" } \\ \text { 忽然(之)間 } & \text { fat1jin4(zil)gaan1 } & \text { "suddenly" } \\ \text { 滾水淥腳(噉) } & \text { gwan2seoi2luk6goek3(gam2) } & \text { "in a hurry" } \\ \text { 一日到黑 } & \text { jat1jat6dou3hak1 } & \text { "all day long" } \\ \text { 一時(之間) } & \text { jat1si4(zilgaan1) } & \text { "for the time being" } \\ \text { 鏈住條頸 } & \text { lin2zyu6tiu4geng2 } & \text { "unwillingly" } \\ \text { 唔怪(之)得 } & \text { m4gwaai3(zil)dak1 } & \text { "that's why" } \\ \text { 無端白事/無情白事 mou4dyunIbaak6si6/mou4cing4baak6si6 } & \text { "without reason" } \\ \text { 三番四次 } & \text { saam1faan1sei3ci3 } & \text { "repeatedly" } \\ \text { 三爬丽撥(噉) } & \text { saam1paa4loeng5but6(gam2) } & \text { "very quickly" } \\ \text { 失驚無神 } & \text { sat1geng1mou4san4 } & \text { "suddenly" } \\ \text { 死死地氣 } & \text { sei2sei2dei6hei3 } & \text { "have no way out" } \\ \text { 食住個勢(就) } & \text { sik6zyu6go3sai3(zau6) } & \text { "by taking advantage } \\ & & \text { of a situation" } \\ \text { 至多(唔係) } & \text { zi3dol(m4hai6) } & \text { "at the worst" }\end{array}$




\subsubsection{Complex Adverbs}

\subsubsection{Monosyllabic Adverbs}

$\begin{array}{lll}\text { 越 } \ldots \text { 越 } \ldots & \text { jyut6 ... jyut } 6 \ldots & \text { "the more ..., the more ..." } \\ \text { 時 } \ldots \text { 時 } \ldots & \text { si } \ldots \text { si } \ldots & \text { "sometimes ..., sometimes ..." }\end{array}$

(The above are those Cantonese adverbs having more or the less the same counterparts in Mandarin Chinese. Only one instance among Cantonese-specific adverbs is a complex monosyllabic adverb.

唔 $\ldots$ 又 $\ldots \quad m 4 \ldots$ jau6 $\ldots \quad$ "not ... but ..."

\subsubsection{Disyllabic Adverbs}

$\begin{array}{lll}\text { 白白 } & \text { baak6baak6 } & \text { "in vain; to no purpose" } \\ \text { 親口 } & \text { canlhau2 } & \text { "(speak) personally" } \\ \text { 親耳 } & \text { can1ji5 } & \text { "(hear) personally" } \\ \text { 親眼 } & \text { canIngaan5 } & \text { "(see) personally" } \\ \text { 親手 } & \text { can1sau2 } & \text { "with one's own hands" } \\ \text { 遲早 } & \text { ci4zou2 } & \text { "some time in the future; some day" } \\ \text { 前後 } & \text { cin4hau6 } & \text { "altogether" } \\ \text { 大大 } & \text { daai6daai6 } & \text { "greatly; enormously" } \\ \text { 一邊...一邊... } & \text { jat1bin1 ...jat1bin1 ... } & \text { "at the same time" } \\ \text { 一一 } & \text { jat1jat1 } & \text { "one by one" } \\ \text { 一面...一面.... } & \text { jat1min6...jat1min6... } & \text { "at the same time" } \\ \text { 日日 } & \text { jat6jat6 } & \text { "daily; regularly" } \\ \text { 日夜 } & \text { jat6je6 } & \text { "day and night" } \\ \text { 遠遠 } & \text { jyun5jyun5 } & \text { "largely" } \\ \text { 略略 } & \text { loek6loek2 } & \text { "a little; a bit; slightly" } \\ \text { 來回 } & \text { loi4wui4 } & \text { "back and forth" } \\ \text { 微微 } & \text { mei4mei2 } & \text { "slightly" } \\ \text { 明明 } & \text { ming4ming4 } & \text { "certainly" } \\ \text { 頻頻 } & \text { pan4pan4 } & \text { "again and again" }\end{array}$




$\begin{array}{lll}\text { 偏偏 } & \begin{array}{l}\text { pinlpin1 } \\ \text { "contrary to speaker's will" } \\ \text { "only" } \\ \text { 時時 }\end{array} & \text { "often; constantly" } \\ \text { 先後 } & \text { si4si4 } & \text { "one after another" } \\ \text { 通通 } & \text { tungltung1 } & \text { "all; entirely" } \\ \text { 漸漸 } & \text { zim6zim2 } & \text { "gradually" } \\ \text { 早早 } & \text { zou2zou2 } & \text { "well in advance" } \\ \text { 足足 } & \text { zuklzuk1 } & \text { "full; as much as" }\end{array}$

(The above are those Cantonese adverbs having more or the less the same counterparts in Mandarin Chinese. Adverbs which are specific to Cantonese are given as follows.)

\begin{tabular}{|c|c|c|}
\hline 差啲 & caaldil & "almost" \\
\hline 齊齊 & cai4cai4 & "together" \\
\hline 次次 & $c i 3 c i 3$ & "every time" \\
\hline 初初 & colcol & "at first" \\
\hline 速速 & cuklcukl & "very quickly; at once" \\
\hline 斷(斷) & dyun6(dyun6) & "absolutely" \\
\hline 快啲 & faai3dil & "quickly" \\
\hline 急急 & gaplgapl & "nearly at once" \\
\hline 輕輕 & heng6heng1 & "slightly" \\
\hline 便...一便.. & jat1bin6...jat1bin6... & "at the same time" \\
\hline 一時... 一時... & jat1si4...jat1si4... & "sometimes; ...sometimes..." \\
\hline 有啲 & jau5dil & "a little bit" \\
\hline 有咐的 & $j u k l d i 1$ & "easily; frequently" \\
\hline 有吓下 & juk1haa5 & "easily; frequently" \\
\hline 勤啲 & kan4di1 & "frequently" \\
\hline 慢慢 & maan6maan 2 & "slowly" \\
\hline \multirow[t]{2}{*}{ 啱啱 } & ngaam 1ngaam1 & "exactly" \\
\hline & & "just" \\
\hline 哩頭...咽頭 $\ldots$ & niltau $4 \ldots$ go $2 \operatorname{tau} 4 \ldots$ & "as soon as" \\
\hline 先先/先前 & $\sin 1 \sin 1 / \sin 1 \operatorname{cin} 4$ & "before; previously" \\
\hline & soeng6haa2 & "almost" \\
\hline 偷 & taulgail & "secretly" \\
\hline 頭尾 & tau4mei5 & "from beginning to end; altogether" \\
\hline & tau4tau2 & "at the beginning" \\
\hline
\end{tabular}




$\begin{array}{lll}\text { 橫掂 } & \text { waang4dim6 } & \text { "anyway" } \\ \text { 掙啲 } & \text { zaangldil } & \text { "almost" }\end{array}$

\subsubsection{Trisyllabic Adverbs}

$\begin{array}{lll}\text { 比較(上) } & \text { bei2gaau3(soeng6) } & \text { "fairly; rather" } \\ \text { 大體(上) } & \text { daai6tai2(soeng6) } & \text { "on the whole" } \\ \text { 大致(上) } & \text { daai6zi3(soeng6) } & \text { "roughly; more or less" } \\ \text { 動不動 } & \text { dung6bat1dung6 } & \text { "frequently" } \\ \text { 非常(之) } & \text { fei1soeng4(zi1) } & \text { "very" } \\ \text { 根本(上) } & \text { gan1bun2(soeng6) } & \text { "at all; simply" } \\ \text { 基本上 } & \text { geilbun2soeng6 } & \text { "basically" } \\ \text { 貿貿然 } & \text { mau6mau6jin4 } & \text { "rashly; hastily" } \\ \text { 十分(之) } & \text { sap6fen1(zi1) } & \text { "very" } \\ \text { 時不時 } & \text { si4bat1si4 } & \text { "sometimes" } \\ \text { 事實上 } & \text { si6sat6soeng6 } & \text { "in fact; actually" } \\ \text { 相當(之) } & \text { soeng1dong1(zi1) } & \text { "quite; fairly; considerably" }\end{array}$

(The above are those Cantonese adverbs having more or the less the same counterparts in Mandarin Chinese. Adverbs which are specific to Cantonese are given as follows.)

\begin{tabular}{|c|c|c|}
\hline 嘭惦聲 & baang4baang2seng1 & "very quickly; at once" \\
\hline 不期然 & bat1kei4jin4 & "unexpectedly" \\
\hline 脆脆哋 & ceoi3ceoi2dei2 & "reluctantly; grudgingly" \\
\hline 遲啲(再) & ci4dil(zoi3) & "later" \\
\hline 大大步 & daai6daai6bou6 & "with wide steps" \\
\hline 大大啖 & daai6daai6daam6 & "at a big mouthful" \\
\hline 大大力 & daai6daai6lik6 & "with a strong force" \\
\hline 大大聲 & daai6daai6seng1 & "very loudly" \\
\hline 大嗱嗱 & daai6laa4laa4 & "as much as" \\
\hline 氹氹羁 & dam4dam4kwaakl & "in a circle" \\
\hline 到頭嫩 & dou3tau4lai4 & "in the end; finally" \\
\hline 分分鐘 & fenlfenlzungl & "at any time" \\
\hline 假假哋 & gaa2gaa2dei2 & "in any case; anyhow" \\
\hline 間(唔)中 & $\operatorname{gaan} 3(\mathrm{~m} 4)$ zung 1 & "occasionally" \\
\hline 雞噉腳 & gailgam 2goek3 & "very quickly" \\
\hline
\end{tabular}




\begin{tabular}{|c|c|c|}
\hline 急急腳 & gaplgaplgoek3 & "in a fast pace" \\
\hline 久唔久 & gau2m4gau2 & "once in a while" \\
\hline 各自各 & gok3zi6gok3 & "respectively" \\
\hline 固然之 & gu3jin4zil & "no doubt" \\
\hline 閒閒哋 & haan4haan2dei2 & "very easily" \\
\hline 係噉意 & hai6gam $2 j i 2$ & "perfunctorily" \\
\hline 好好(吡) & hou2hou2(dei2) & "well" \\
\hline 一步步 & jat1bou2bou6 & "step by step; progressively" \\
\hline 一時時 & jat1si2si4 & "occasionally" \\
\hline 現兒淣 & jin6douldoul & "in cash" \\
\hline 越嚟越 & jyut6lai4jyut6 & "more and more" \\
\hline 擒擒青 & kam4kam2cengl & "quickly" \\
\hline 嗱(嗱)臨 & laa4(laa4)lam4 & "very quickly; at once" \\
\hline 嗱嗱聲 & laa4laa2sengI & "very quickly; at once" \\
\hline 唔使問 & m4sai2man6 & "undoubtedly" \\
\hline 唔使審 & $m 4$ sai2sam2 & "undoubtedly" \\
\hline 唔掙在 & m4zaanglzoi6 & "never mind" \\
\hline 面對面 & $\min 6 d e o i 3 \min 6$ & "face-to-face" \\
\hline 無情情 & mou4cing4 cing 4 & "without reason" \\
\hline 無端端 & mou4dyun I dyunl & "without reason" \\
\hline 無意中 & mou4ji3zungl & "not intentionally; by chance" \\
\hline 無髪檂 & mou4laallaal & "without reason" \\
\hline 啱(啱)先 & ngaaml(ngaam1) $\sin 1$ & "just" \\
\hline \multicolumn{3}{|c|}{ 啱啱好/啱啱線 ngaam1ngaam1hou2/ngaam Ingaam 1 sin3 } \\
\hline 眼白白 & ngaan5baak6baak6 & "helplessly" \\
\hline \multicolumn{2}{|c|}{ 耐不耐/而唔耐 noi6bat1noi2/noi6m4noi2 } & "once in a while" \\
\hline 十成十 & sap6sing4sap6 & "surely" \\
\hline 衰衰哋 & seoilsoeildei2 & "nevertheless" \\
\hline 施施然 & silsiljin4 & "leisurely; unhurriedly" \\
\hline 偷偷哋 & taultauldei2 & "secretly" \\
\hline 䁪吓下眼 & zaam2haa5ngaan5 & "in an instant" \\
\hline 借啲意 & $z e 3 d i 1 j i 2$ & "by making an excuse" \\
\hline 整整吓 & zing2zing $2 h a a 2$ & "gradually" \\
\hline 靜雞雞 & zing6gailgail & "quietly" \\
\hline 轉吓下眼 & zyun2haa5ngaan5 & "in an instant" \\
\hline 轉(個)頭 & zyun2(go3)tau4 & "later" \\
\hline
\end{tabular}




\subsubsection{Polysyllabic Adverbs}

$\begin{array}{lll}\text { 口口聲聲 } & \text { hau2hau2senglseng1 } & \text { "(say) again and again" } \\ \text { 陸陸續續 } & \text { luk6luk6zuk6zuk6 } & \text { "in succession" } \\ \text { 無緣無故 } & \text { mou4jyun4mou4gu3 } & \text { "without reason" } \\ \text { 無時無刻 } & \text { mou4si4mou4hak1 } & \text { "all the time" }\end{array}$

(The above are those Cantonese adverbs having more or the less the same counterparts in Mandarin Chinese. Adverbs which are specific to Cantonese are given as follows.)

\begin{tabular}{|c|c|c|}
\hline 大大話話 & daai6daai6waa6waa6 & "approximately" \\
\hline 大啖大啖 & daai6daam6daai6daam6 & "at a big mouthful" \\
\hline 多多少少 & doldo1siu2siu2 & "to a certain extent" \\
\hline 快快脆脆 & faai3faai3ceoi3ceoi3 & "quickly" \\
\hline 快手快腳 & faai3sau2faai3goek3 & "quickly" \\
\hline 加加埋埋 & gaalgaalmaai4maai4 & "altogether" \\
\hline 夾手夾腳 & gaap3sau2gaap3goek3 & "jointly" \\
\hline 急急忙忙 & gaplgap1mong4mong4 & "in a hurry; in a haste" \\
\hline 幾時得嚟 & gei2si4dak1lai4 & "not (within a period of time)" \\
\hline 口快快(噉) & hau2faai3faai3(gam2) & "without hesitation" \\
\hline 開口埋口 & hoilhou2maai4hou2 & "(keep) talking over the same thing" \\
\hline 好好醜醜 & hou 2 hou 2 cau 2 cau 2 & "in any case; whatever happens" \\
\hline 好唔好都 & hou $2 m 4$ hou 2 doul & "in any case; whatever happens" \\
\hline 一次又一次 & jat1ci3jau6jat1ci3 & "time and again; repeatedly" \\
\hline 一手一腳 & jat1sau2jat1goek3 & "by oneself" \\
\hline 有理無玨 & jau5lei5mou5lei5 & "regardless of the consequences" \\
\hline 依時(依候) & jilsi4(ji1hou6) & "punctually" \\
\hline 卡那(卡那) & kaa3laal(kaa3laal) & "alternatively" \\
\hline 臨急臨忙 & lam4gap Ilam4mong4 & "at the last moment" \\
\hline 臨時臨急 & lam4si4lam4gap1 & "at the last moment" \\
\hline 哩哩啦啦 & li4li1laa4laa4 & "very quickly" \\
\hline 落手落月 & lok6sau2lok6goek3 & "by oneself; in person" \\
\hline 唔多唔少 & $m 4 d o 1 m 4 \operatorname{siu} 2$ & "to a certain extent" \\
\hline 唔經唔覺 & $m 4$ ging1 1 4gok3 & "without knowing consciously" \\
\hline & $m 4$ senglm4seng 1 & "quietly" \\
\hline 實与 & sat6ngaa4sat6ci2 & "certainly" \\
\hline
\end{tabular}




$\begin{array}{lll}\text { 實實在在 } & \text { sat6sat6zoi6zoi6 } & \text { "in fact" } \\ \text { 時時刻刻 } & \text { si4si4haklhakl } & \text { "all the time" } \\ \text { 借頭借路 } & \text { ze3tau4ze3lou6 } & \text { "by making an excuse" } \\ \text { 自自然然 } & \text { zi6zi6jin4jin4 } & \text { "naturally" }\end{array}$

\subsection{RECURRENT PATternings OF AdVERbS}

Some internal structures are found to recurrently accompany disyllabic, trisyllabic and polysyllabic adverbs. Among these adverbs, trisyllabic adverbs display a greater variety of common structures than the others. Among these common structures, reduplication is given greater prominence than others, which is to be discussed in greater detail in the next section.

\subsubsection{With Directional Complement 嚟 lai4}

$\begin{array}{lll}\text { 到頭黎 } & \text { dou3tau4lai4 } & \text { "in the end; finally" } \\ \text { 幾時得黎 } & \text { gei2si4dak1lai4 } & \text { "not (within a period of time)" }\end{array}$

\subsubsection{With Directional Complement 上 soeng6}

$\begin{array}{lll}\text { 比較(上) } & \text { bei2gaau3(soeng6) } & \text { "relatively" } \\ \text { 大體(上) } & \text { daai6tai2(soeng6) } & \text { "on the whole" } \\ \text { 大致(上) } & \text { daai6zi3(soeng6) } & \text { "roughly; more or less" } \\ \text { 根本(上) } & \text { gan1bun2(soeng6) } & \text { "at all; simply" } \\ \text { 基本上 } & \text { geilbun2soeng6 } & \text { "basically" } \\ \text { 事實上 } & \text { si6sat6soeng6 } & \text { "in fact; actually" }\end{array}$

\subsubsection{With Negation Adverb}

$\begin{array}{lll}\text { 唔掙在 } & \text { m4zaang1zoi6 } & \text { "never mind" } \\ \text { 唔使問 } & m 4 \text { sai2man6 } & \text { "undoubtedly" } \\ \text { 唔使審 } & m 4 \text { sai2sam2 } & \text { "undoubtedly" }\end{array}$




\subsubsection{With 中 zung1}

$\begin{array}{lll}\text { 間(唔)中 } & \text { gaan3(m4)zungl } & \text { "occasionally" } \\ \text { 無意中 } & \text { mou4ji3zungl } & \text { "not intentionally; by chance" } \\ \text { 而唔中 } & \text { noi6m4zung1 } & \text { "once in a while" }\end{array}$

\subsubsection{With 腳 goek3}

Two trisyllabic adverbs denoting quickness of an action are associated with the word 腳 goek3.

$\begin{array}{lll}\text { 雞噉腳 } & \text { gailgam2goek3 } & \text { "very quickly" } \\ \text { 急急腳 } & \text { gaplgaplgoek3 } & \text { "at a fast pace" }\end{array}$

\subsubsection{With 意 $j i 2$}

$\begin{array}{lll}\text { 係噉意 } & \text { hai6gam2ji2 } & \text { "perfunctorily" } \\ \text { 借啲意 } & z e 3 d i 1 j i 2 & \text { "by making an excuse" }\end{array}$

\subsubsection{With 然jin4}

不期然

貿貿然

施施然

\section{bat1kei4jin4}

mau6mau6jin4

silsiljin4 "unexpectedly"

"rashly; hastily"

"leisurely; unhurriedly"

\subsubsection{With 之 $z i 1$}

$\begin{array}{lll}\text { 非常(之) } & \text { feilsoeng4(zil) } & \text { "very" } \\ \text { 十分(之) } & \text { sap6fen1(zil) } & \text { "very" } \\ \text { 相當(之) } & \text { soengldongl(zil) } & \text { "quite; fairly" } \\ \text { 固然之 } & \text { gu3jin4zil } & \text { "no doubt" }\end{array}$




\subsubsection{AA + 聲 seng1}

Two trisyllabic adverbs end in a word literally denoting "sound". It is associated with reduplicated adverbs meaning "very quickly" and a change of tone to the high-rising tone on the second syllable.

$\begin{array}{lll}\text { 㰻澎聲 } & \text { baang4baang2seng1 } & \text { "very quickly; at once" } \\ \text { 嗱嗱聲 } & \text { laa4laa2seng1 } & \text { "very quickly; at once" }\end{array}$

\subsubsection{A-唔 $m 4-\mathrm{A}$}

$\begin{array}{lll}\text { 久唔久 } & \text { gau2m4gau2 } & \text { "once in a while" } \\ \text { 而唔而 } & \text { noi6m4noi2 } & \text { "once in a while" }\end{array}$

\subsubsection{A-不 bat1-A}

$\begin{array}{lll}\text { 動不動 } & \text { dung6batldung6 } & \text { "frequently" } \\ \text { 時不時 } & \text { si4bat1si4 } & \text { "sometimes" } \\ \text { 而不耐 } & \text { noi6bat1noi2 } & \text { "once in a while" }\end{array}$

\subsubsection{V/ADJ + 的 $d i 1$}

This word 啲 dil occurs mainly with disyllabic adverbs. It carries a diminutive meaning of "a little bit".

$\begin{array}{lll}\text { 差啲 } & \text { caaldi1 } & \text { "almost" } \\ \text { 遲啲(再) } & \text { ci4dil(zoi3) } & \text { "later" } \\ \text { 快啲 } & \text { faai3di1 } & \text { "quickly" } \\ \text { 有啲 } & \text { jau5di1 } & \text { "a little bit" } \\ \text { 有啲 } & \text { jukldil } & \text { "easily; frequently" } \\ \text { 勤啲 } & \text { kan4di1 } & \text { "frequently" } \\ \text { 掙啲 } & \text { zaangldil } & \text { "almost" }\end{array}$




\subsubsection{V+吓haa5}

The suffix 吓 haa5 is attached to the end of the verb or inserted between the verb and its object.

$\begin{array}{lll}\text { 有吓下 } & \text { juklhaa5 } & \text { "easily; frequently" } \\ \text { 整整吓 } & \text { zing2zing2haa2 } & \text { "gradually" } \\ \text { 睴下下眼 } & \text { zaam2haa5ngaan5 } & \text { "in an instant" } \\ \text { 轉吓眼 } & \text { zyun2haa5ngaan5 } & \text { "in an instant" }\end{array}$

\subsubsection{Verb + Object}

$\begin{array}{lll}\text { 偷雞 } & \text { taulgail } & \text { "secretly" } \\ \text { 轉(個)頭 } & \text { zyun2(go3)tau4 } & \text { "later" } \\ \text { 鏈住條頸 } & \text { lin2zyu6tiu4geng2 } & \text { "unwillingly" } \\ \text { 食住個勢(就) } & \text { sik6zyu6go3sai3(zau6) } & \text { "by taking advantage of a favourable } \\ & & \text { situation" }\end{array}$

\subsubsection{5 $\mathrm{ADJ}_{\mathrm{i}}+\mathrm{ADJ}_{\mathrm{i}}+$ 哋 dei2}

This suffix - 哋 dei2 is used productively with trisyllabic adverbs. The suffixation of this affix results in a change of tone to the high-rising tone on the second adjective, unless the tone on which is already a high level tone or a high-rising tone.

$\begin{array}{lll}\text { 脆脆哋 } & \text { ceoi3ceoi2dei2 } & \text { "reluctantly; grudgingly" } \\ \text { 假假哋 } & \text { gaa2gaa2dei2 } & \text { "in any case; anyhow" } \\ \text { 閒閒哋 } & \text { haan4haan2dei2 } & \text { "very easily" } \\ \text { 好好(哋) } & \text { hou2hou2(dei2) } & \text { "well" } \\ \text { 衰衰哋 } & \text { seoilseoildei2 } & \text { "nevertheless" } \\ \text { 偷偷哋 } & \text { taultau1dei2 } & \text { "secretly" }\end{array}$

\footnotetext{
${ }^{9}$ The tone of the suffix - 吓 is changed to a high-rising one.
} 


\subsubsection{6 親 $\operatorname{can1}+$ Noun (denoting body part responsible for speech or actions)}

$\begin{array}{lll}\text { 親口 } & \text { can1hau2 } & \text { "(speak) personally" } \\ \text { 親耳 } & \text { can1ji5 } & \text { "(hear) personally" } \\ \text { 親眼 } & \text { can1ngaan5 } & \text { "(see) personally" } \\ \text { 親手 } & \text { can1sau2 } & \text { "with one's own hands" }\end{array}$

\subsubsection{Antonymous Combinations}

$\begin{array}{lll}\text { 遲早 } & \text { ci4zou2 } & \text { "some time in the future; some day" } \\ \text { 前後 } & \text { cin4hau6 } & \text { "altogether" } \\ \text { 日夜 } & \text { jat6je6 } & \text { "day and night" } \\ \text { 來回 } & \text { loi4wui4 } & \text { "back and forth" } \\ \text { 先後 } & \text { sin1hau6 } & \text { "one after another" } \\ \text { 上下 } & \text { soeng6haa2 } & \text { "almost" } \\ \text { 頭尾 } & \text { tau4mei5 } & \text { "from beginning to end; altogether" } \\ \text { 橫掂 } & \text { waang4dim6 } & \text { "anyway" }\end{array}$

\subsection{REDUPLICATED ADVERBS}

As previously mentioned, adverbs have shown some recurrent patterns. Some reduplicated adverbs are recurrently used with 哋 dei2, 聲 seng1，不 bat1 and 唔 $m 4$. In fact, the manifestations of reduplication in Cantonese adverbs fall into a wide range of systematic patterns. These patterns are to be discussed in the following.

\subsubsection{AA Form}

Some disyllabic adverbs, typically formed from adjectives, are accompanied by reduplication. As Matthews and Yip (1994:184) point out, "the reduplication may result in a changed tone on the second adjective." The tone being changed to is usually the high-rising tone (Tone 2); only one adverb is found with the high level 
tone (Tone 1) on its second adjective. Examples are shown below. The shaded syllables of the adverbs are found to undergo the change in tone.

\begin{tabular}{|c|c|c|}
\hline 白白 & baak6baak6 & "in vain; to no purpose" \\
\hline 大大 & daai6daai6 & "greatly; enormously" \\
\hline - - & jatljat1 & "one by one" \\
\hline 日日 & jat6jat6 & "daily; regularly" \\
\hline 遠遠 & jyun5jyun5 & "not at all" \\
\hline 略略 & loek6loek2 & "a little; a bit; slightly" \\
\hline 微微 & mei4mei2 & "slightly" \\
\hline 明明 & ming4ming4 & "certainly" \\
\hline 頻頻 & pan4pan4 & "again and again" \\
\hline 偏偏 & pin1pin1 & "contrary to speaker's will; just" \\
\hline 時時 & si4si4 & "often; constantly" \\
\hline 通通 & tungltungl & "all; entirely" \\
\hline 漸漸 & zim6zim2 & "gradually" \\
\hline 早早 & zou2zou2 & "well in advance" \\
\hline 足员 & zuklzukl & "full; as much as" \\
\hline 齊齊 & cai4cai4 & "together" \\
\hline 次次 & $c i 3 c i 3$ & "every time" \\
\hline 初初 & colcol & "at first" \\
\hline & cuklcukl & "very quickly; at once" \\
\hline 斷(斷) & dyun6(dyun6) & "absolutely" \\
\hline 急急 & gaplgapl & "nearly at once" \\
\hline 輕軴 & heng6heng1 & "slightly" \\
\hline 好好(哋) & hou2hou2(dei2) & "well" \\
\hline & maan6maan2 & "slowly" \\
\hline 砛 & ngaamlngaaml & "exactly; just" \\
\hline & $\sin 1 \sin 1$ & "before; previously" \\
\hline & tau4tau2 & "at the beginning" \\
\hline
\end{tabular}

\subsubsection{AAB Form}

The reduplicated adverbs with 哋 dei2 and 聲 sengl fall into this category. Other examples of this class are shown as follows. The syllable with change of tone to the high-rising tone is shaded. 


\begin{tabular}{|c|c|c|}
\hline 貿貿然 & mau6mau6jin4 & "rashly; hastily" \\
\hline 大大步 & daai6daai6bou6 & "with wide steps" \\
\hline 大大啖 & daai6daai6daam6 & "at a big mouthful" \\
\hline 大大力 & daai6daai6lik6 & "with a strong force" \\
\hline 大大聲 & daai6daai6seng1 & "very loudly" \\
\hline 氹氹緙 & dam4dam4kwaakl & "in a circle" \\
\hline 分分鐘 & fenlfenlzung1 & "at any time" \\
\hline 急急腳 & gaplgaplgoek3 & "in a fast pace" \\
\hline 擒擒青 & kam4kam2ceng 1 & "quickly" \\
\hline 嗱(嗱)臨 & laa4(laa4)lam4 & "very quickly; at once" \\
\hline 啱(啱)先 & ngaaml(ngaam1) sin 1 & "just" \\
\hline 啱啱好/啱啱線 & ngaam1ngaam1hou2/ngaam1ng & "just right" \\
\hline 施施然 & silsiljin4 & "leisurely; unhurriedly" \\
\hline 整整吓 & zing2zing2haa2 & "gradually" \\
\hline 靜雞雞 & zing6gailgail & "quietly" \\
\hline
\end{tabular}

\subsubsection{ABA Form}

The reduplicated adverbs with 不 bat1 and 唔 $m 4$ fall into this category.

Other examples are shown as follows.

$\begin{array}{lll}\text { 各自各 } & \text { gok3zi6gok3 } & \text { "respectively" } \\ \text { 越嚟越 } & \text { jyut6lai4jyut6 } & \text { "more and more" } \\ \text { 面對面 } & \text { min6deoi3min6 } & \text { "face-to-face" } \\ \text { 十成十 } & \text { sap6sing4sap6 } & \text { "surely" } \\ \text { 一次又一次 } & \text { jat1ci3jau6jat1ci3 } & \text { "again and again" }\end{array}$

\subsubsection{ABB Form}

This form might occasionally result in the change of tone on the first element of the reduplicated part. The changed tone is typically the high-rising tone.

$\begin{array}{lll}\text { 大嗱嗱 } & \text { daai6laa4laa4 } & \text { "as much as" } \\ \text { 口快快(噉) } & \text { hau2faai3faai3(gam2) } & \text { "without hesitation" }\end{array}$




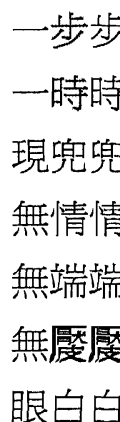

jat1bou2bou6

jat1si2si4

jin6dauldau1

mou4cing4cing 4

mou4dyun1dyun 1

mou4laallaal

ngaan5baak6baak6 "step by step; progressively"

"occasionally"

"in cash"

"without reason"

"without reason"

"without reason"

"helplessly"

\subsubsection{AABB Form}

$\begin{array}{ll}\text { 口口聲聲 } & \text { hau2hau2senglseng1 } \\ \text { 陸陸續續 } & \text { luk6luk6zuk6zuk6 } \\ \text { 大大話話 } & \text { daai6daai6waa6waa6 } \\ \text { 多多少少 } & \text { doldolsiu2siu2 } \\ \text { 快快脆脆 } & \text { faai3faai3ceoi3ceoi3 } \\ \text { 加加埋埋 } & \text { gaalgaalmaai4maai4 } \\ \text { 急急忙忙 } & \text { gaplgap1mong4mong4 } \\ \text { 好好醜醜 } & \text { hou2hou2cau2cau2 } \\ \text { 哩哩啦啦 } & \text { li4lillaa4laa4 } \\ \text { 實實在在 } & \text { sat6sat6zoi6zoi6 } \\ \text { 時時刻刻 } & \text { si4si4hak1hak1 } \\ \text { 自自然然 } & \text { zi6zi6jin4jin4 }\end{array}$

"(say) again and again"

"in succession"

"approximately"

"to a certain extent"

"quickly"

"altogether"

"in a hurry; in a haste"

"in any case; whatever happens"

"very quickly"

"in fact"

"all the time"

"naturally"

\subsubsection{ABAB Form}

$\begin{array}{ll}\text { 大啖大啖 } & \text { daai6daam6daai6daam6 } \\ \text { 卡那(卡那) } & \text { kaa3laa1(kaa3laa1) } \\ \text { 唔聲唔聲 } & m \text { 4seng1m4seng1 }\end{array}$
"at a big mouthful"
"alternatively"
"quietly"

\subsubsection{ABAC Form}

$\begin{array}{ll}\text { 無緣無故 } & \text { mou4jyun4mou4gu3 } \\ \text { 無時無刻 } & \text { mou4si4mou4hak1 } \\ \text { 快手快腳 } & \text { faai3sau2faai3goek3 } \\ \text { 夾手夾腳 } & \text { gaap3sau2gaap3goek3 } \\ \text { 好唔好都 } & \text { hou2m4hou2doul }\end{array}$

"without reason"

"all the time"

"quickly"

"jointly"

"in any case; whatever happens" 


$\begin{array}{lll}\text { 一手一腳 } & \text { jat1sau2jatlgoek3 } & \text { "by oneself" } \\ \text { 依時(依候) } & \text { ji1si4(jilhou6) } & \text { "punctually" } \\ \text { 臨時臨急 } & \text { lam4si4lam4gap1 } & \text { "at the last moment" } \\ \text { 臨急臨忙 } & \text { lam4gap1lam4mong4 } & \text { "at the last moment" } \\ \text { 落手落腳 } & \text { lok6sau2lok6goek3 } & \text { "by oneself; in person" } \\ \text { 唔多唔少 } & m 4 \text { dolm4siu2 } & \text { "to a certain extent" } \\ \text { 唔經唔覺 } & m 4 g i n g 1 m 4 g o k 3 & \text { "without knowing consciously" } \\ \text { 實牙實齒 } & \text { sat6ngaa4sat6ci2 } & \text { "definitely; undoubtedly" } \\ \text { 借頭借路 } & \text { ze3tau4ze3lou6 } & \text { "by making an excuse" }\end{array}$

\subsubsection{ABCB Form}

開口埋口 hoilhau2maai4hau2

"(keep) talking over the same thing"

有理無理 jau5lei5mou5lei5

"regardless of the consequences"

\subsection{CO-ORDINATE PAIRS}

Some adverbs must occur in co-ordination with another adverb of the same form.

(1) 越...越...jyut6...jyut6... "the more... the more..."

我越諗越声癷。

ngo5 jyut6 nam2 jyut6 hing3

I more think more angry

"The more I think, the more I get angry."

(2) 時...時...si4 ...si4 ... "sometimes... sometimes..." 佢時喊時笑。

keoi5 si4 haam3 si4 siu3

$\mathrm{s} /$ he sometimes cry sometimes laugh

"S/he cried partly and laughed partly." 
(3) 一路...一路...jat1lou6...jat1lou6... "at the same time; simultaneously" 我一路睇一路笑。

ngo5 jat1lou6 tai2 jat1lou6 siu3

I simultaneously read simultaneously laugh

"I kept on laughing while reading."

(4) 一時...一時...jat1si4...jat1si4 ... "sometimes... sometimes..."

啲天氣二時好熱, 一時好凍。

dil tin1 hei3 jat1si4 hou2 jit6 jat1si4 hou2 dung 3

$\mathrm{M}$ weather sometimes very hot sometimes very cold

"The weather sometimes became very hot but sometimes became very cold."

\subsection{ANTONYM PAIRS}

Some adverbs are in fact antonyms. These antonymous adverbs are typically formed by a minimal pair of adjectives or verbs with opposite meanings.

$\begin{array}{lll}\begin{array}{l}\text { 好在 } \\ \text { 弊在 }\end{array} & \begin{array}{l}\text { hou2zoi6 } \\ \text { bai6zoi6 }\end{array} & \begin{array}{l}\text { "fortunately" } \\ \text { "unfortunately" }\end{array} \\ \text { 好日 } & \text { hou2jat6 } & \text { "rarely; seldom" } \\ \text { 無日 } & \text { mou5jat6 } & \text { "all the time" } \\ \text { 有得 } & \text { jau5dak1 } & \text { "it is possible to" } \\ \text { 無得 } & \text { mou5dakl } & \text { "it is not possible to" }\end{array}$

It is worth noting that a pair of adverbs, 有幾何 jau5gei2ho2 and 無幾何 mou5gei2ho2 which appear to be antonymous superficially turn out to have the same meaning "rarely; seldom". However, their usage is different; 有幾何 jau5gei2ho2 is required to occur in rhetorical question but 無幾何 mou5gei2ho2 is not (see also Chapter 6, section 6.2.2). 
（5）你有幾何洯叫?

nei5 jau5gei2ho2 lai4 aal

you rarely come FP

"You rarely come here."

(6) 你無幾何洯。

nei5 mou5gei2ho2 lai4

you rarely come

"You rarely come here."

\subsection{SUMMARY}

Over half of the Cantonese adverbs are disyllabic. Trisyllabic adverbs are characteristic of various common structures. Polysyllabic adverbs are characteristic of different patterns of reduplication. Some adverbs must occur in co-ordination with themselves. Some adverbs are formed by antonymous adjectives and verbs that make them opposite in meaning. The following table gives a summary of the distribution of Cantonese adverbs with respect to their structural complexity and syllabicity.

\begin{tabular}{l|c|c|c|c|c}
\hline \multirow{2}{*}{ Syllabicity } & \multicolumn{2}{|c|}{ Simple Adverbs } & \multicolumn{2}{c|}{ Complex Adverbs } & \multirow{2}{*}{ Total } \\
\cline { 2 - 5 } & $\mathbf{C M}^{\mathrm{a}}$ & $\mathbf{C S}^{\mathrm{a}}$ & $\mathbf{C M}$ & $\mathbf{C S}$ & \\
\hline Monosyllabic Adverbs & 27 & 21 & 2 & 1 & 51 \\
\hline Disyllabic Adverbs & 164 & 129 & 25 & 25 & 343 \\
\hline Trisyllabic Adverbs & 6 & 30 & 12 & 51 & 99 \\
\hline Polysyllabic Adverbs & 2 & 16 & 4 & 30 & 52 \\
\hline
\end{tabular}

Table 1: Simple and complex adverbs in Cantonese

Note a: Refer to Chapter 1, section 1.4.1 for the description of the abbreviations CM and CS.

Note b: The single entry in Appendix 1 至多(味/唔係) zi3dol (mai6/m4hai6) is divided into separate entries 至多(咪) zi3dol (mai6) and 至多(唔係) zi3dol (m4hai6), thus increasing the total number of adverbs (cf. Appendix 1) by one. 


\section{Semantic Classification of Adverbs}

\subsection{INTRODUCTION: PROPOSALS FOR CLASSIFICATION IN THE LITERATURE}

Several studies are devoted to adverbs in English as well as Mandarin Chinese, and in each study the adverbs are somehow classified using different criteria. The number of categories varies, and even more importantly, the names chosen for a category may also differ, so, to avoid confusion for those acquainted with the literature, some important previous classifications will be considered before presenting the one used here. The different proposals for both English and Mandarin Chinese will be discussed separately below.

\subsubsection{English classification of adverbs}

Previous studies of semantic classification of English adverbs include Greenbaum (1969), Jackendoff (1972), Thomason and Stalnaker (1973), Lehrer (1975a), Bellert (1977), McConnell-Ginet (1982), Erust (1984), Vendler (1984), Swan (1988), Nakamura (1997), Ramat and Ricca (1998), and Wyner (1998). In their semantic theory of adverbs, Thomason and Stalnaker (1973) have divided adverbs into two classes, namely sentence adverbs and predicate adverbs. However, as Bellert (1977) notes, not all the sentence adverbs can uniformly be described in such a way.

Jackendoff's method (semantic-paraphrase analyses) has much influence on

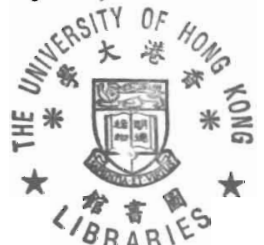


subsequent works $^{10}$ (Nakamura, 1997:249). It is therefore worthwhile to give a sketchy account of Jackendoff's classification. Jackendoff divides English adverbs into four classes, taking into consideration both the semantic and distributional properties of adverbs. The four classes are (1) manner adverbs, (2) subject-oriented adverbs, (3) speaker-oriented adverbs, and (4) the merely class. ${ }^{11}$ He defines manner adverbs as occurring in auxiliary and final position, both subject-oriented adverbs and speaker-oriented adverbs as occurring in initial and auxiliary position, and the merely class as occurring only in auxiliary position. Each of these sub-classes has its own semantic paraphrase. Jackendoff paraphrases (1) - (4) as (5) - (8), respectively.

(1) John dropped his cup of coffee clumsily.

(2) Cleverly, John spilled the beans.

(3) Happily, John won the game. ${ }^{12}$

(4) Frankly, John lied to Bill.

(5) The manner in which John dropped his cup of coffee is clumsy.

(6) It was clever of John to spill the beans.

(7) It is happy (to me) that John won the game.

(8) *It is frank (to me) that John lied to Bill.

Owing to the fact that sometimes the semantic-paraphrase is not compatible with the speaker-oriented adverb, like frankly, as illustrated in (8), Bellert justifies her attempt to refine Jackendoff's classification of adverbs and provides more accurate paraphrases of them. In particular, she further divides the speaker-oriented adverbs into the following five sub-classes (see Nakaruma, 1997:250-252; for description of Bellert's analyses).

\footnotetext{
${ }^{10}$ Not only do Jackendoff's paraphrases of adverb subcategories have direct impact on later accounts of sub-classification of adverbs, but also indirectly form the basis to elucidate other linguistic phenomena, such as to show that the line between semantics and pragmatics is not at all clear-cut (Lehrer, 1975b) and to explain how sentences are related to the events they describe, possibly in two ways which arise from two distinct paraphrases for manner adverbials and speaker-oriented adverbials (Moore, 1993).

${ }_{11}$ Jackendoff does not give any semantic property of this merely class (e.g. merely, utterly, virtually, etc.).

${ }^{12}$ The example (3) is ambiguous, meaning either "it was happy of John to win the game" or (7) as given.
} 

A. Evaluative adverbs (luckily, fortunately, happily, surprisingly, etc.)
B. Modal adverbs (probably, possibly, certainly, surely, evidently, etc.)
C. Domain adverbs (logically, mathematically, morally, aesthetically, etc.)
D. Conjunctive adverbs (however, nevertheless, hence, therefore, firstly, finally, etc.)
E. Pragmatic adverbs (frankly, sincerely, honestly, briefly, precisely, roughly, etc.)

In short, in dealing with English adverbs, linguists tend to subdivide adverb classes on semantic grounds by providing paraphrases of them.

\subsubsection{Chinese classification of adverbs}

In Chinese, different grammarians classify adverbs into smaller groups in accordance with different criteria. Generally speaking, previous literature classifies Chinese adverbs mainly in two ways, one of which is on the basis of grammatical meanings expressed by adverbs, and the other of which is based on position of adverb in a clause or sentence. Grammarians like Wang Li (1959:267-268), Chao Yuen Ren (1968:780-795) and Zhu Dexi (1982:195-201) adopt the former approach in dealing with classification of adverbs whereas Charles Li and Sandra Thompson (1989:319-339) adopt the latter.

Let me begin with the classification of adverbs by position. Li and Thompson (1989:320) divide adverbs into the following three sub-classes:

1. Movable Adverbs

2. Nonmovable Adverbs

3. Postverbal Adverbials

This classification is established on the basis of adverb placement.

Movable adverbs occur either before the subject or after it but before the verb. Semantically, they are further divided into time adverbs and attitude adverbs. 
Nonmovable adverbs occur only after the subject but before the verb. They comprise manner adverbs and nonmanner adverbs according to semantic characteristics.

Functionally, movable adverbs modify the entire clause so they are also called sentential adverbs, whereas nonmovable adverbs modify the verb, adjective or adverb phrases in which they occur.

Postverbal adverbials occur after the verb and specify the duration or extent of the action expressed by the predicate. In fact, examples of this type do not involve adverbs at all; instead they are made up of noun phrases consisting of a number, a classifier (if one is required) and a noun. Hence, absolutely speaking, this type of "adverbs" must not be treated as a subdivision of adverbs. Here are some examples of each adverb subcategory given by Li and Thompson.

(9) Movable adverb of time

a. 剛岡我不舒服, 現在好多了。

gānggang wŏbu shüfu xiànzài hăo duōle

just-now I not comfortable now good much FP

"Just now I didn't feel well but I feel better now."

b. 我剛剛不舒服, 現在好多了。

wǒ gānggang bu shüfu xi ànzài hăo duō le

I just-now not comfortable now good much FP

"Just now I didn't feel well but I feel better now."

(10) Movable adverb of attitude

a. 誠然張三不開心。

xiănrán zhāngsān bu gāoxing

obviously Zhangsan not happy

"Obviously, Zhangsan is not happy."

b. 張三誠然不開心。

Zhāngsān xiănrán bu gāoxing

Zhangsan obviously not happy

"Obviously, Zhangsan is not happy." 
(11) Nonmovable manner adverb

他快快地走。

tākuài-kuài-de zŏu

he quickly walk

"He walked quickly."

(12) Nonmovable nonmanner adverb

他它經走了

tāyijing zŏu le

he already leave FP

"He has already left."

\begin{tabular}{|l|l|c|c|c|}
\hline & Chinese Adverb Sub-classes & Wang (1959) & Chao (1968) & Zhu (1982) \\
\hline 1 & Adverb of Affirmation & & $\checkmark$ & \\
\hline 2 & Adverb of Contingency & $\checkmark$ & $\checkmark$ & \\
\hline 3 & Adverb of Degree & $\checkmark$ & $\checkmark$ & $\checkmark$ \\
\hline 4 & Adverb of Evaluation & & $\checkmark$ & \\
\hline 5 & Interrogative Adverb & & $\checkmark$ & \\
\hline 6 & Modal Adverb & $\checkmark$ & & \\
\hline 7 & Linking Adverb & $\checkmark$ & & \\
\hline 8 & Adverb of Manner & $\checkmark$ & $\checkmark$ & \\
\hline 9 & Adverb of Negation & $\checkmark$ & $\checkmark$ & \\
\hline 10 & Adverb of Place & & $\checkmark$ & \\
\hline 11 & Adverb of Possibility & $\checkmark$ & & \\
\hline 12 & Adverb of Quantity & & $\checkmark$ & \\
\hline 13 & Adverb of Scope & $\checkmark$ & $\checkmark$ & $\checkmark$ \\
\hline 14 & Adverb of Time & $\checkmark$ & $\checkmark$ & $\checkmark$ \\
\hline
\end{tabular}

Table 2: The classification of adverbs in the Chinese literature

Table (2) illustrates different labels used by different grammarians in classifying adverbs according to their grammatical meanings. This table demonstrates two major problems in such kind of classification. Firstly, names of sub-classes have not been standardized. There has not been any consensus in naming sub-classes of adverbs; linguists use their own terminology in doing so. In fact, despite different names used, they tend to denote more or less the same grammatical meaning. Secondly, the total number of sub-classes is different. Chao gives as many as 
eleven sub-classes of adverbs whereas Zhu gives as few as four. To make matters worse, as Zhang Yisheng (2000:17) notes, the same adverb is subsumed under one class by one grammarian but under another class by another grammarian!

There can be infinite number of names coined by linguists. However, it should be borne in mind that a word can be classified as an adverb even without knowing its sub-class so long as it exhibits all the syntactic properties an adverb proper should have. Subsuming it under a particular class is merely a means to highlight the common properties shared by members of this sub-class. The name or label of this sub-class is therefore vital in the discussion of shared semantic and syntactic properties. In dealing with Cantonese adverbs, I assume the following taxonomy in which Cantonese adverbs are divided into seven sub-classes according to grammatical meaning.

\subsection{CONJUNCTIVE ADVERB (C)}

Conjunctive adverbs serve a linking function amongst verb/adjective phrases and amongst clauses. They are distinguished from conjunctions as they have to be put only after the subject (conjunctions can be put either before the subject or after it). A conjunctive adverb can function alone like 咪 mai6 "then", 先(至) $\sin 1$ (zi3) "then", 就 zau6 "then", etc., or function in concert with the same adverb or another adverb, for example, 又...又 ...,jau6...jau6... "at the same time", 固然之...只不 過...gu3jin4zi1 ...zi2bat1gwo3... "no doubt ...only...", 一...就...jat1 ...zau6... "as soon as...then...", 一旦...就...jat1daan6...zau6... "once ... then...", etc.

\section{(13) 著多啲衫, 你咪唔凍囖。} zoek 3 dol dil saam1 nei5 mai6 $\mathrm{m} 4$ dung 3 lo 1 wear many PL clothes you then not cold FP "Wear more clothes and you don't feel cold." 
(14) 噏得出就噏。

ap1-dak1-ceot1 zau6 ap1

say-COMP-out as-soon-as say

"You just say what you can say."

(15) 佢做嘢文快文好。

keoi5 zou6je5 jau6 faai3 jau6 hou2

s/he work also fast also good

"He works efficiently and effectively."

(16) 噉做固然之好, 只市過係怕佢唔鍾意啫。

gam2 zou6 gu3jin4zi1 hou2 zi2bat1gwo3 hai6 paa3 keoi5 $\mathrm{m} 4$ zunglji3 zel

in-such-way do no-doubt good only be fear s/he not like FP

"No doubt, it is good to do it this way. However, we are only afraid of making him unhappy."

\subsection{DEGREE ADVERB}

Degree adverbs can form a scale of degree. As Klein (1998) persuasively puts it when discussing adverbs of degree in general,

[t] he specification of an adverb of degree ... indicates a range on the scale of the appropriate dimension in which the quality concerned can be placed. We may try to order the adverbs along the scale, but it is evident that groups of adverbs point to roughly the same range of the scale. Terribly, highly, fantastically and awfully all indicate a very high degree, and although differences with other adverbs, such as rather or very, can be stated, the internal ordering of the former group cannot easily be decided upon.

(Klein, 1998:14)

Klein's viewpoints will be used as a reference throughout this section.

Cantonese degree adverbs can be graded in the scale according to, roughly, the highest degree such as 至 $z i 3$ "most", an absolutely high degree such as 咁(鬼) gam3(gwai2) "very", a relatively high degree such as 哽 ngang3 "even more", an absolutely intermediate degree such as 算 syun 3 "fairly", a relatively intermediate 
degree such as 比較(上) bei2gaau3(soeng6) "rather", an absolutely low degree such as 有啲 jau $5 d_{11}$ "a little bit", or a relatively low degree such as 輕輕 heng6heng1 "slightly". Diagrammatically, the scale of degree and degree adverbs ascribed to the scale is illustrated in Figure (2).

In fact, the notions of absolute and relative adverbs of degree exist in both Mandarin Chinese and English. Wang Li (1959:268-276) has divided Mandarin Chinese adverbs of degree into these two groups. Lu Jianming and Ma Zhen (1999), and Han Rongzhu (2000) further rationalise such division by pointing out the distribution of absolute and relative degree adverbs in different sentence types, and their pragmatic differences with respect to modality and presupposition respectively; that is, semantic categorisation is justified both syntactically and pragmatically. Though the Longman Grammar of Spoken and Written English (1999) does not clearly state the terms "absolute" and "relative", it gives the definition of degree adverbs in absolute and relative senses:

They [adverbs of degree] can be used to mark that the extent or degree is either greater or less than usual [absolute sense] or than that of something else in the neighbouring discourse [relative sense]. (1999:554)

\begin{tabular}{|c|c|c|c|c|}
\hline Absolute & & Scale of Degree & & Relative \\
\hline- & 4 & -...- HIGHEST -........ & $\longrightarrow$ & 至 $z i 3$ "most" \\
\hline 咁(鬼) & 4 & -_.-- HIGH -.. & $\longrightarrow$ & 哽 ngang3 \\
\hline $\operatorname{gam} 3$ (gwai2) & & & & "even more" \\
\hline "so" & & & & \\
\hline 相當(之) & 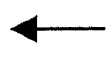 & HIGH-INTERMEDIATE & $\rightarrow$ & 靈舍 ling4se3 \\
\hline soengldongl(zil) & & & & "particularly" \\
\hline "considerably" & & & & \\
\hline 算 syun3 "fairly" & 4 & |-- INTERMEDIATE --- & 一 & 比較(上) \\
\hline & & & & bei2gaau3(soeng6) \\
\hline & & & & "rather" \\
\hline 有啲 jau $5 d i 1$ & $\longleftarrow$ & |_....... LOW -- & $\longrightarrow$ & 輕輕 heng6hengl \\
\hline "a little bit" & & & & "slightly" \\
\hline
\end{tabular}

Figure 2: Degree adverbs and degree scale 
Some Chinese linguists tend to emphasise the role of degree adverbs as a whole in modifying other elements (Chao Yuen Ren, 1968; Zhu Dexi, 1982; He Yang, 1994; Yang Yun, 1999; among others). On the other hand, some linguists tend to focus on a particular adverb of degree and its use (Luo Qingsong, 1995; Zhao Lijiang, 1998; Liu Yuanman, 1999; among others). This sort of discussion on the use of particular adverbs is important as it reveals that Mandarin Chinese degree adverbs differ from Cantonese ones with respect to the same individual one.

It is generally agreed that the adverb of degree 太 $t a \grave{i}$ "too" in Mandarin Chinese expresses two grammatical meanings: (1) high degree, used in admiration; (2) excessive degree, used in indicating unfortunate or undesirable events. ${ }^{13}$ Cantonese adopts the same adverb from Mandarin Chinese but uses it differently in two respects: (1) the degree adverb 太 taai3 is optionally accompanied by 過 gwo3 to form 太 (過)taai3(gwo3); (2) the degree adverb 太(過)taai3(gwo3) is mainly used for excessive degree. In other words, the same adverb in Cantonese differs from that in Mandarin Chinese in both form and meaning. In indicating a high degree, Cantonese tends to use 好 hou2, 幾咁/幾鬼 gei2gam3/gei2gwai2, etc. rather than 太(過)taai3(gwo3).

\section{High Degree}

(17) 啲湯幾咁好味啊 !

dil tong1 gei2gam3 hou2mei6 aa3

PL soup very delicious FP

"The soup is so delicious."

\footnotetext{
${ }^{13}$ Lü Shuxiang 呂叔湘 (1999) 《現代漢語八百詞》"太[副]1.表示程度過頭, 多用於不如意的 事情。句未常帶'了'。2.表示程度高・多用於讚歎。" p.526; Zhonggwo Shewui Kexue Yuyan Yanjiusuo Cidian Bianjishi (1999) 《現代漢語詞典》"太[副]a.表示程度過分； b.表示程度極高(用於讚靯)。 " p.1219.
} 
（18）啲湯太(過)好味啊！(Note: The sentence is still acceptable but rare.)

dil tongl taai3(gwo3) hou 2 mei6 aa3

PL soup very delicious FP

"The soup is so delicious."

Cf. (19) 這個湯的味道味太美了。

zhèi gè tâng de wèidào täi mĕi le

this M soup POSS taste very beautiful FP

"This soup is so delicious."

\section{$\underline{\text { Excessive Degree }}$}

(20) 哩條題目太難塩!

nil tiu4 tai4muk6 taai3 naan4 laa3

this $\mathrm{M}$ question too difficult FP

"This question is too difficult."

\section{Cf. (21) 這道題太難了。 zhè dào tít täi nán le this $\mathrm{M}$ question too difficult FP \\ "This question is too difficult."}

Similarly, though the degree adverb 夠 gò in Mandarin Chinese is used to indicate that an element it modifies sufficiently satisfies speaker's demand on its level or indicate a high degree ${ }^{14}$, Cantonese 夠 gau3 is not a degree adverb at all; rather, it is a modal adverb carrying strong emotional colouring.

(22) 佢怨我? 我夠怨佢咯!

keoi5 jyun 3 ngo5 ngo5 gau3 jyun3 keoi5 lok3

$\mathrm{s} /$ he blame me I also blame him/her FP

"She blames me? But I blame her too!"

${ }^{14}$ Lü Shuxiang 呂叔湘 (1999) 《現代漢語八百詞》"夠[副]1.修飾形容詞, 表示達到一定標準。 形容詞只能是積極意義的, 不能是相應的反義詞。2.修飾形容詞, 表示程度很高。形容詞可以 是積極意義的。句尾多加'的'或'了'。"p.235. 


\subsection{MANNER ADVERB (M)}

Manner adverbs characterise the action indicated by the verb. It is worth noting that about 10 manner adverbs are subsumed under a smaller category named "repetition". These manner adverbs are typically used to indicate a repetitive action or event. They do not form a separate class of their own, being distinct from manner adverbs because they are like manner adverbs in that they specify how an action is done: an action is done once again.

(23) 佢文試食零食。

keoi5 jau6si3 sik6 ling4sik6

$\mathrm{s}$ /he again eat snacks

"She eats snacks again."

(24) 佢至番西次唔聽人勸。

keoi5 saam 1faan1sei3ci3 m4 teng1 jan 4 hyun 3

$\mathrm{s} /$ he again-and-again not listen-to people persuade

"She did not listen to us again and again."

The majority of manner adverbs suggests a variety of ways of undergoing an action. For instance, an action can occur at a fast pace as described by the adverb 擒摛青 kam4kam2cengl "quickly". An interesting observation is that for those manner adverbs specifying how fast an action is taken place, they might consist of the morphemes 聲 sengl "sound" and 腳 goek3 "leg" as in 嘭塏聲 baang4baang2seng1 "very quickly", 嗱嗱聲 laa4laa2seng1 "very quickly", 急急腳 gaplgaplgoek3 "at a fast pace", 雞噉腳 gailgam2goek3 "very quickly", etc.

(25) 仲唔嘭膨聲出去揾佢?

zung $6 m 4$ baang4baang2seng1 ceot 1 heoi 3 wan 2 keoi5

still not very-quickly go-out look-for him/her

"Why don't you go out and look for him at once?" 
(26) 我哋嗱挐聲做埋啲嘢喇。

ngo5dei6 laa4laa2seng1 zou6-maa4 dil je5 laa1

we very-quickly do-COMP PL thing FP

"Let's finish the work quickly."

(27) 佢急急腳走過噄。

keoi5 gap1gap1goek3 zau2 gwo3 lai4

$\mathrm{s} /$ he in-a-fast-pace walk across come

"He came here quickly."

(28) 你做七㝬噋腳走過噄?

nei5 zou6matl gailgam2goek3 zau2 gwo3 lai4

you why very-quickly walk across come

"Why did you come here so quickly?"

There are also adverbial characterizations having to do with the volition and intent of the agent in respect of the execution of the action, as depicted by the adverbs 專登 zyun1dang1 and 夾硬 gaap3ngaang2. Furthermore, an action may occur in succession as shown by 連氣 lin4hei3, or in a random manner by 笠亂 lap6lyun2, or in secret by 偷偷吔 taultauldei2.

(29) 我專登噄探你嗎。

ngo5 zyun1dang1 lai4 taam 3 nei5 gaa3

I deliberately come visit you FP

"I come to see you deliberately."

(30) 夾硬迫人去。

gaap3ngaang2 bik1 jan4 heoi3

unyieldingly force people go

"People are forced to come not willingly."

(31) 佢連氣食咗三大碗麵。

keoi5 lin4hei3 sik6-zo2 saam1 daai6 wun2 min6

$\mathrm{s} /$ he in-succession eat-ASP three big M noodles

"He ate three big bowls of noodles successively." 
(32) 哩啲說話唔好笠䇏乚講。

nil dil syut3waa6 m4hou2 lap6lyun2 gong2

that PL words do-not casually say

"Don't say something thoughtlessly."

(33) 佢偷偷哋出咗街。

keoi5 taultau1dei2 ceot1-zo2 gaail

$\mathrm{s} /$ he secretly go-out-ASP street

"She went out secretly."

There are a few manner adverbs in Cantonese that have the structure "- jat1 'one' (+ measure word) + noun", for example, 一口氣 jat1hau2hei3 "without a break", 一輪嘴 jat1leon2zeoi2 "without a break", 一眼 jat1ngaan5 "in a glimpse", 一心 jat1sam1 "wholeheartedly", 一手 jat1sau2 "single-handed", 一手一腳 jat1sau2jatlgoek3 "by oneself", etc. Apparently, they look like noun phrases functioning as adverbials. However, it is argued that they are genuine adverbs. If they were noun phrases, they would mean what their components literally mean. In fact, they mean quite differently from their literal meanings as in the following examples. Note that their literal meanings are given in the gloss.

(34) 佢㷫起噄二向氣唱咗十幾隻歌。

keoi5 hing3-hei2lai4 jat1hau2hei3 coeng3zo2 sap6-gei2 zek3 gol

$\mathrm{s} /$ he excited-COMP without-a-break sing-ASP ten-something $\mathrm{M}$ songs

"He was so excited that he sang about ten songs without a break."

(35) 佢一輪嘴講哂啲嘢出嚟。

keoi5 jat1leon2zeoi2 gong2-saai3 dil je5 ceot1lai4

s/he by-one-mouth say-COMP PL thing go-out

"She spoke all the things without a break."

(36) 佢一眼認出我。

keoi5 jat1ngaan 5 jing6ceot1 ngo5

s/he by-one-eye recognise me

"She recognized me in a glimpse." 
(37) 我一心想教書。

ngo5 jat1sam1 soeng2 gaau3syu1

I by-one-heart want-to teach

"I want to be a teacher wholeheartedly."

(38) 佢一手就捻起張橙。

keoi5 jat1sau2 zau6 ling1 hei2 zoeng1 dang3

s/he in-one-hand EMPH pick-up M chair

"He picked up the chair single-handedly."

(39) 個蛋糕我二手二焀做嘅。

go3 daan6goul ngo5 jat1sau2jat1goek3 zou6 ge3

$M$ cake I in-one-hand-one-leg do FP

"I made the cake by myself."

It does not make sense to sing ten songs in one breath as in (34), to recognise a person in one eye as in (36), and to make a cake in one hand and one leg as in (39). Further, though it is true that a person has one mouth, i.e. 一輪嘴 jat1leon2zeoi2, and one heart, i.e. 一心 jat1sam1, these literal meanings of their components differ dramatically from the actual meanings as in (35) and (37), respectively. Even if the literal meaning seems close to the actual meaning as in (38), 一手 jat1sau2 is not a grammatical noun phrase. The structure of a noun phrase in both Mandarin Chinese and Cantonese requires a measure word to be inserted between the numeral and the head noun (whereas English does not).

$$
\begin{aligned}
& \text { (40) 一隻手 } \\
& \text { jat1 zek3 sau2 } \\
& \text { one M hand } \\
& \text { "one hand" }
\end{aligned}
$$

Hence, as shown in the above, 一隻手 jat1 zek3 sau2 "one hand" is a grammatical noun phrase whereas 一手 jat1sau2 is not; 一手 jat1sau2 is an adverb instead. 


\subsection{MODAL ADVERB (ML, MA, MV)}

Modal adverbs refer to those adverbs that project speaker's judgement, evaluation and strong emotions on a situation described by the predicate. Not every linguist uses exactly the term "modal adverb" in referring to the same group of adverbs. Chao Yuen Ren (1968:781-786), for instance, uses the terms "adverb of evaluation" and "adverb of contingency". In fact, what he means by "adverb of evaluation" is the second kind of modal adverbs to be described in the following, and what he means by "adverb of contingency" is the first kind.

Modal adverbs can be further divided into three categories. Firstly, a group of modal adverbs (ML) might express "evidentiality" (Chafe, 1986), that is, possibility, likelihood or certainty on the situation. The level of doubt or certainty is signified by the use of different adverbs; to put another way, these epistemic adverbs form a scale of probability (Hoffner, Cantor, and Badzinski, 1990:220), where adverbs of high certainty have the probability of one and adverbs of lower certainty have the probability less than one.

Some modal adverbs tend to indicate a very high level of certainty such as 絕對 zyut6deoi3, 十成十 sap6sing4sap6, 實行 sat6hang4 etc. A less certain (but still highly certain) situation is expressed by adverbs like 九成 gau2sing4, 多數 do1sou3, 唔見得 $m 4 g i n 3 d a k l$, etc. A situation which is partly certain and partly doubtful is expressed by adverbs like 唔多唔少 $m 4 d o 1 m 4 \operatorname{siu} 2$, 少不免 siu2bat 1 min 5 , 未必 mei6bitl etc. A more doubtful situation is expressed by adverbs such as 怕(且) paa3(ce2), 玩吓手 waan2haa5sau2, 或者 waak6ze2, etc.

(41) 佢噉嘅樣, 卋成卋輸咗喇。 keoi5 gam2 ge3 joeng2 sap6sing4sap6 syu1-zo2 laal s/he such-way GE look surely lose-ASP FP "He looks so frustrated. Surely, he lost the game." 


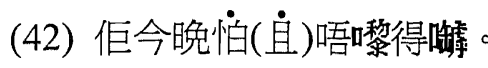

keoi5 gam1maan1 paa3(ce2) m4 lai4-dak1 laa3

$\mathrm{s} /$ he tonight perhaps not come-COMP FP

"Perhaps, she couldn't come tonight."

It is interesting to note that the modal adverb 包 baaul "certainly" seems to be derived from its verbal counterpart of the same homonymous form meaning "guarantee". In other words, as Traugott and and König (1991. Cited in Kryk-Kastovsky, 1997:320) puts in one of the semantic-pragmatic tendencies proposed by them, "meanings tend to become increasingly situated in the speaker's subjective belief/state/attitude toward the situation". In their study of sentence adverbs in the languages of Europe, Ramat and Ricca (1998:243-244) also impressively points out that "[t]he semantic development usually goes from the world being talked about to the views on that world uttered by the speaker in her/his act of speaking", and called this process of "a change towards more abstract meanings" subjectification (see also Powell, 1992; Peters, 1994).

For some evidence to this claim, Conradie (1993:93-95) has reported that the modal adverb glo "allegedly" in Afrikaans is derived from the verb glo "believe", and thus convincingly demonstrated that subjectification refers to "any process causing a lexical item to develop a subjective meaning or extending the membership of modals in the lexicon". I can observe this shift of meaning from a more concrete one as expressed by a verb to a more abstract one as expressed by an adverb in sentences like (43) and (44), respectively:

(43) Verb 包 baaul "guarantee"

包你滿意。

baaul nei5 bun5ji3

guarantee you satisfy

"(I) guarantee that you must be satisfied (with it)." 
(44) Adverb 包 baaul "certainly"

今日包落雨。

gamljat6 baau1 lok6jyu5

today certainly rain

"It certainly rains today."

Secondly, a group of modal adverbs (MA) on the other hand reflects speaker's attitude towards the content independent of its epistemological status. Some attitudinal adverbs appear to show speaker's surprise on the content, for instance, 點 (不)知 $\operatorname{dim} 2$ (bat1)zil, 竟然 ging2jin4, 居然 geoiljin4, etc. Other adverbs express speaker's determination in taking on an action, for example, 一於 jat1jyul, 有理無 理 jau5lei5mou5lei5, 幾大 gei2daai2, etc.

(45) 佢點(六)知唔嚟得啝。 (surprise)

keoi5 dim2(bat1)zi1 m4 lai4-dak1 wo4 s/he unexpectedly not come-COMP FP

"Unexpectedly, she couldn't come."

(46) 幾大都要去。 (determination)

gei2daai2 doul jiu3 heoi3

no-matter-what EMPH want-to go

"No matter what happens, I need to go."

Cantonese has some modal adverbs which signal expectations of some kind. They range from 都係 doulhai6, 情願 cing4jyun2, etc., which show a particular expectation a speaker holds to 硬係 ngaang2hai6, 偏偏 pin1pin1, etc., which indicates a conflict, suggesting that a fact goes beyond what one might have expected.

(47)諗諗吓, 都係唔去好啲喎。 (expectation)

nam2-nam2-haa2 dou1hai6 $m 4$ heoi3 hou2dil wo3

think-think-ASP had-better not go better FP

"On second thought, I had better not to go there." 
(48) 本書硬係買唔到。

(contrary to expectation)

bun2 syul ngaang2hai6 maai5m4dou2

$\mathrm{M}$ book contrary-to-expectations buy-not-COMP

"Unfortunately, the book is difficult to find."

Furthermore, some of these modal adverbs express a concession like 話晒 waa6saai3, 好好醜醜 hou2hou2cau2cau2, etc. whereas others express speaker's strong emotions or emphasis like 直情 zik6cing4, 先(至) $\sin 1$ (zi3), etc. Some modal adverbs express speaker's suggestion/persuasion such as 千祈 cin1kei4, 何苦 ho4fu2, 最好 zeoi3hou2, etc. Some modal adverbs express a contrary fact such as 反爲 faan2wai4，又jau6，實則 sat6zak1, etc. Some modal adverbs express speaker's instantaneous understanding of the situation such as 唔怪(之)得 m4gwaai3(zi1)dak1, 原來 jyun4loi4, 難怪 naan4gwaai3, etc.

(49) 你暲吓佢喇, 佢話晒都係你細佬。(concession) nei5 bong2-haa5 keoi5 laal keoi5 waa6saai3 doul hai6 nei5 sai3lou2 you help-ASP him/her FP s/he after-all rather be your brother "After all, he is your brother so you should help him."

(50) 你先(至)係, 我唔係! (emphasis) nei5 $\sin 1$ (zi3) hai6 ngo5 $\mathrm{m} 4$ hai6 you EMPH be I not be "It is you. Not me!"

(51) 手祈唔好講俾人知。 (suggestion) cin1kei4 m4hou2 gong2 bei2 jan4 zi1 for-heaven's-sake do-not say to people know "For heaven's sake, don't tell others."

(52) 佢實則唔係咁懶茄。 (contrary fact) keoi5 sat6zak1 m4 hai6 gam3 laan5 ge2 s/he actually not be so lazy FP "Actually, she is not as lazy as you thought." 
(53) 唔怪(之)得佢唔去喇, 原來佢有事。 (understanding) m4gwaai3(zil)dak1 keois $\mathrm{m} 4$ heoi3 laal jyun4loi4 keoi5 jau5si6 that-is-why s/he not come FP originally s/he have-something-to-do "That's why he doesn't come. He has got something to do."

Both epistemic and attitudinal adverbs are generally loosely attached to the sentences. They are used to orient the hearer towards the statement in which they occur and explain to the hearer how the statement is to be taken. One of the characteristics of these adverbs is that they can be positioned quite freely in a sentence without changing their meaning (Huang, 1975:26).

Thirdly, a few modal adverbs (MV) appear to present the point of view of the speaker, for instance, 嗱埋 laa2maai4 "generally speaking", 計正 gai3zeng3 "as a rule", 聽講(話) tenglgong2(waa6) "by hearsay", etc., expressing a vague viewpoint or hearsay. Other members of this sub-class might also suggest that the source of knowledge does not lie in the speaker alone but all the external, objective facts being taken together, as in 大體上 daai6tai2soeng6 "on the whole" and 總算 zung2syun 3 "all things considered".

(54) 哩隊籃球隊嘅球員個個挰埋都有兩米以上。

nil deoi6 laam4kau4deoi2 ge3 kau4jyun4 go3go3 laa2maai4 doul jau5 loeng5mail ji5soeng6 this $\mathrm{M}$ basketball-team GE player everyone generally-speaking all have two-metre above

"Generally speaking, each player of this basketball team is over two-metre tall."

\subsection{NEGATION AdVERB (N)}

Though negation adverbs, as their name suggests, are used to negate the existence of an action or state named by the predicate, each of them negates in a slightly different way. For example, 未嘗 mei6soeng4 is used in negative sentence only. 
(55) 佢末嘗唔係一個好人。

keoi5 mei6soeng $4 \mathrm{~m} 4$ hai6 jat1 go3 hou 2 jan 4

$\mathrm{s} /$ he not not be one $\mathrm{M}$ good person

"He is a good person after all."

(56) 噉對佢揫講, 未當唔係一件好事叫。

gam2 deoi3 keoi5 lai4 gong2 mei6soeng4 m4 hai6 jat1 gin6 hou 2 si6 aal

in-such-way to him/her come say not not be one M good matter FP

"To her, it's not a bad thing."

It is worth noting that I consider 唔多 $m 4 d o 1$, 唔使 $m 4$ sai 2 , 唔慌 $m 4$ fong 1 and 唔好 $m 4$ hou 2 as one single unit, though others might argue that they should be two separate words i.e. negation adverb + verb/adjective rather than one word. I suspect that 多 dol "very much" and 使 sai2 "need" as in 唔多 m4dol and 唔使 m4sai2, respectively, have been obsolete. They are replaced by another words 好 hou 2 "very" and 要 jiu3 "need" nowadays.

(57) 佢好覞。

keoi5 hou 2 leng3

s/he very beautiful

"She is very beautiful."

(58) *佢多靚。

(59) 佢要去。

keoi5 jiu3 heoi3

s/he need-to go

"She needs to go."

（60）*佢使去。

Hence, I do not consider 多 dol and 使 sai2 words at all but bound morphemes only. Furthermore, though 慌 fong1 "worry" and 好 hou2 "very" are undoubtedly words in Cantonese, their meanings are changed in combination with 唔 $m 4 .^{15}$

${ }^{15}$ See Chao and Mui (1999) for a somewhat different approach in arguing that 唔好 $m 4$ hou2 is a single form. 
(61) 佢唔慌係好人。

keoi5 m4fong1 hai6 hou2 jan4

$\mathrm{s} /$ he absolutely-not be good person

"He is absolutely not a good person."

Cf. (62) 我唔慌佢唔噄。

ngo5 $\mathrm{m} 4$ fongl keoi5 ng4 lai4

I not worry s/he not come

"I don't worry that he doesn't come."

(63) 你唔好喊喇。

nei5 $\mathbf{m} 4$ hou 2 haam3 laal

you don't cry FP

"Please don't cry."

Cf. (64) 中史都唔好讀咖。

zunglsi2 doul m4 hou2 duk6 ge2

Chinese-history EMPH not good study FP

"Chinese History is not easy to study."

\subsection{SCOPE ADVERB (S)}

Scope adverbs specify the scope in which an action named by the predicate takes effect. The scope can be further classified into nine kinds in Cantonese. The first sort of scope involves all the things or people affected by the action, as expressed by, for example, 正蹦呤 ham6baang6laang6, 一律 jatlleot6, 通通 tungltung1, etc. The second kind of scope expresses the fact that part, but not all, of the things or people are affected by the action, as in 淨係 zing6hai6, 偏偏 pin1pin1, etc.

(65) 啲嘢我岀蹋哙拎晒返屋企。

di1 je5 ngo5 ham 6baang6laang6 ling1-saai3 faan1 uk1kei2

PL thing I all bring-COMP return home

"I take all the things and bring them home." 
(66) 屋企淨係得我一個人。

uklkei2 zing6hai6 dak1 ngo5 jat1 go3 jan4

home only have I one M person

"Only I am at home, no one else."

Apart from giving the "whole-or-part" distinction, some scope adverbs signal the limit or extent that things or people are affected by the action. Hence, the third kind of scope refers to the upper limit or the largest extent as in 頂多 ding4do1, 極 其量 gik6kei4loeng6, etc. whereas the fourth kind of scope refers to the lower limit or the least extent as in 起碼 hei2maa5, 點止 dim2zi2, 座底 zo6dai2, etc. The fifth kind of scope refers to an addition on things or people involved in the action, as expressed by 亦(都)jik6(dou1), 又 jau6, etc.

(67) 佢頂多十五歲。

keoi5 ding2do1 sap $6 m 5$ seoi3

s/he at-most fifteen years-old

"He is at most fifteen."

(68) 哩度座底三百人。

nildou6 zo6dai2 saamlbaak 3 jan 4

here at-least three-hundred people

"There are at least three hundred people."

(69) 佢主要讀經濟, 會計亪(都)識啲嗎。

keoi5 zyu2jiu3 duk6 ginglzai3 wui6gai3 jik6(dou1) sik1 dil gaa3

s/he mainly study economics accounting also know PL FP

"He majors in Economics but he also knows some accounting."

Other devices of expressing scope signal the quantity of entities affected in the action or state. Thus, the sixth kind of scope indicates the total number of things or people affected by the action as in 成 seng4, 足足 zuklzukl, 加加埋埋 gaalgaalmaai4maai4, etc. whereas the seventh kind of scope indicates an approximation as in 約莫 joek3mok2, 大大話話 daai6daai6waa6waa6, etc. 
(70) 哩本書成三百蚊駕。

nil bun2 syul seng4 saam1baak3 man1 gaa3

this $\mathrm{M}$ book as-much-as three-hundred dollars FP

"This book is as much as three hundred dollars."

(71) 任約莫三十歲。

keoi5 joek3mok2 saam1sap6 seoi3

s/he approximately thirty years-old

"He is about thirty something."

The eighth kind of scope expresses the fact that things or people are almost affected by the action as in 差啲 caaldi1, 幾乎 geilfu4, 咁滯 gam3zai6, etc. The ninth kind of scope indicates the spatial scope; there are altogether three scope adverbs in this category, namely 隨處 ceoi4cyu3, 隨地 ceoi4dei6 and 到處 dou3cyu3.

(72) 我差啲認唔到佢。

ngo5 caa1di1 jing6-m4-dou2 keois

I almost recognise-not-COMP him/her

"I almost cannot recognise him."

(73) 你唔好將啲哩予遀處掉喇!

nei5 m4hou2 zoeng1 dil je5 ceoi4cyu3 deu6 laa1

you do-not PREP PL thing everywhere put FP

"Don't put the things everywhere."

Previous studies on scope adverbs in Mandarin Chinese put much emphasis on 都 $d \overline{o u}$. This is because this adverb causes ambiguity when two or more elements come before or after it and can be modified by it, and it will thus be difficult to determine which element the scope adverb 都 $d \overline{o u}$ is in fact modifying (Chao Yuen Ren, 1968:780. See also Zhu Dexi, 1982:195-196).

Recent research reveals that besides being used as a scope adverb, 都 $d \overline{o u}$ can also be a modal adverb (see Shi Xiyao, 1990; Chen Zhijiao, 1996; Guo Chungui, 
1997; Yang Mansheng, 1998; Jiang Yan, 1998; Wang Hong, 1999; among others for discussion). Wang (1999) demonstrates three common sentence structures in which this modal adverb 都 $d \bar{o} u$ occurs while Guo (1997) identifies that it expresses three emotional colourings: dissatisfaction, persuasion and regret.

Cantonese adopts the same adverb 都 doul from Mandarin Chinese with some changes in meaning; there is one particular meaning of this Cantonese adverb that is missing in its Mandarin Chinese counterpart. As a scope adverb, it means the first kind, "all", and the fifth kind of scope, "also".

(74) 人都走晒咯, 做鬼做馬咩!

jan4 dou1 zau2-saai3 lok3 zou6gwai2zou6maa5 me1 people all gone-COMP FP no-point-in-working FP "People have all left. There is no point in working any more."

(75) 你去咩? 我都去喎。 nei5 heoi3 mel ngo5 doul heoi3 wo3 you come FP I also come FP "You will come there? Me too!"

As a modal adverb, sometimes it expresses speaker's reservations in admitting something. This particular use of the Cantonese adverb 都 doul is missing in Mandarin Chinese, where another adverb 還 hái is used instead. In most of the cases, the modal adverb 都 doul is simply used to express emphasis.

(76) 哩條裙都唔錯。

nil tiu4 kwan4 dou1 $\mathrm{m} 4 \mathrm{co} 3$

this $M$ skirt after-all nice

"This skirt is nice after all."

\section{Cf. (77) 這條裙子還不錯。} zhèi tiáo qúnzi hái bùcuò this M skirt after-all nice "This skirt is nice after all." 
(78) 我都話唔得閒咯!

ngo5 doul waa6 $m 4$ daaklhaan4 lok3

I EMPH say not available FP

"I have told you that I was not available."

\subsection{Time AdVerb (T)}

Time adverbs are those adverbs referring to the time or frequency or period of time which the sentence specifies. They can express different temporal meanings, according to Wang Li (1959:274-280), who divides time adverbs into eight distinct temporal senses. Lu Jianming and Ma Zhen (1999), on the other hand, divide time adverbs into two major groups of definite and non-definite temporal specification on the grounds that very few time adverbs are fixed in referring to a definite time in the past, present or future. They note that approximately $80 \%$ time adverbs are not fixed in this way.

Cantonese time adverbs behave exactly like their Mandarin Chinese counterparts in this regard. Time adverbs like 跟手 ganlsau2 "next" and 即刻 ziklhaakl "immediately" can be used to denote both future and past, and both present and past, respectively.

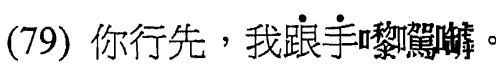
nei5 haang4 sin1 ngo5 gan1sau2 lai4 gaa3 laa3 you walk first I next come FP FP

"You set out first. I will follow in a minute."

(80) 㧽晚佢食完飯, 跟手就搭車返咗廣州。

go2 maan1 keoi5 sik6-jyun4 faan6 gan1sau2 zau6 daap3 ce1 faan1-zo2 gwong2zau1 that night s/he eat-COMP rice next as-soon-as take-a-ride-on car return-ASP Guangzhou

"That night, after he had the meal, he took a ride on a bus and returned Guangzhou." 
(81) 你等我, 我而家即刻黎。

nei5 dang2 ngo5 ngo5 ji4gaal zik1haak1 lai4

you wait-for I I now immediately come

"Just wait for me for a while. I come at once."

(82) 佢尋日俾人打劫, 即刻報咗警。

keoi 5 cam4jat6 bei2 jan4 daa2gip3 zik1haak1 bou3-zo2 ging2

$\mathrm{s}$ /he yesterday by people rob immediately reported-ASP police

"As soon as he was robbed, he called the police."

However, time adverbs such as 試過 si3gwo3 "once" and 第二時 dai6ji6si4 "later" are used to denote, respectively, past and future only.

(83) 我試過驚到唔識出聲。

ngo5 si3gwo3 geng1-dou 34 sikl ceotlseng1

I before frightened-COMP not know speak

"Once I was so frightened that I did not know how to respond."

(84) 我哋第二時再傾喇。

ngo5dei6 dai6ji6si4 zoi3 kingl laal

we later again talk FP

"We can talk to each other later."

In the present study, Cantonese adverbs are therefore further divided into three types: past/present/future time adverbs, frequency time adverbs and duration time adverbs. Past/present/future time adverbs are not distinguished separately but grouped as a whole so as to avoid implying that an adverb should refer to a definite time. Frequency time adverbs refer to the frequency at which an action occurs, for example, 有啲 juk1di1 "frequently", 無幾何 mou5gei2ho2 "rarely", etc.

(85) 佢有啲就鬧人。

keoi5 jukldil zau6 naau6 jan4

s/he frequently as-early-as scold people

"She scolded others frequently." 
(86) 你無幾何嚟探我。

nei5 mou5gei2ho2 lai4 taam 3 ngo5

you rarely come visit I

"You seldom come to see me."

Duration time adverbs refer to the duration for which an action lasts, for instance, 枕長 zam2coeng4 "for a long time", 轉下眼 zyun2haa 5ngaan5 "in an instant", etc.

(87) 我枕言住係醫院。

ngo5 zam2coeng4 zyu6 hai2 jiljyun2

I for-a-long-time live at hospital

"I have been living in hospital for a long time."

(88) 佢轉叶眼唔見咗人。

keoi5 zyun2haa5ngaan5 m4-gin3-zo2 jan4

$\mathrm{s} /$ he in-an-instant not-see-ASP people

"He just disappeared in an instant."

\subsection{FINAL REMARKS}

The above classification of adverbs into sub-categories is largely based on meanings. This way of classifying adverbs has been adopted by previous studies as mentioned in the introductory section of this chapter. It is not without its own shortcoming though. Nakaruma (1997:252) has pointed out that it is difficult to explain why semantic paraphrases hold. Moreover, the fact that an adverb expresses more than one grammatical meaning ${ }^{16}$ makes the borderline among different sub-types of adverbs blurred; hence, subcategorisation based on meanings is not at all robust and has to be critically reconsidered (Ramat and Ricca 1998:247).

\footnotetext{
${ }^{16}$ For some evidence on the polysemous nature of adverbs, see, for example, Tao Hongyin (2000) who states that a class of adverbs (conglai 從來, shizhong 始終, xianglai 向來, yixiang一向, quanran 全然, genben 根本, benlai 本來, and yizhi 一直) in Chinese have two kinds of grammatical meanings, time and assertiveness where the latter, subjective meaning is evoked by the former, objective meaning.
} 
As Yang Rongxiang (2000:53) notes, "it is suggested that the further classification of adverbs into sub-categories should observe not only the standard of semantic features but also that of combining functions. The two standards should permeate each other and prove each other." In other words, Yang emphasises that any meaning-based classification of adverbs into smaller groups has to be syntactically substantiated. ${ }^{17}$

One of the "combining functions" Yang mentions refers to the fact that different adverb sub-classes tend to modify different elements, for instance, verb phrase, adjective phrase, even the whole clause. This will be discussed in the next chapter. It will be shown that the sub-classes of adverbs do have different choices in modifying other elements; thus this further classification of adverbs is justified syntactically (see Chapter 5, section 4).

\begin{tabular}{|c|c|c|}
\hline Adverb Subclasses & Grammatical Meanings (if any) & Examples \\
\hline Conjunctive Adverb & Linking & 就, 咪 \\
\hline \multirow[t]{5}{*}{ Degree Adverb } & Highest & 至 \\
\hline & High & 咁(鬼), 哽 \\
\hline & High-intermediate & 相當(之), 靈舍 \\
\hline & Intermediate & 算，比較(上) \\
\hline & Low & 有啲，輕輕 \\
\hline \multirow[t]{7}{*}{ Manner Adverb } & Repetitive action & 又試, 三番四次 \\
\hline & Pace of action & 擒擒青，搻嗱聲 \\
\hline & Action in deliberation & 專程, 特登 \\
\hline & Action in succession & 連氣，連隨 \\
\hline & Action in randomness & 笠亂，亂(咁) \\
\hline & Involuntary action & 夾硬，監粗 \\
\hline & Action in secret & 偷偷地 , 靜雞雞 \\
\hline \multirow[t]{4}{*}{ Modal Adverb } & \multicolumn{2}{|l|}{ Level of Certainty/Doubt } \\
\hline & $\diamond \quad$ Highly Certain & 認真, 十成十 \\
\hline & $\diamond \quad$ Less Certain & 九成，多數 \\
\hline & \multicolumn{2}{|c|}{ Partly certain and partly doubtful 唔多唔少，少不强 } \\
\hline
\end{tabular}

17 "劃分副詞次類, 在沒有找到單純依據語法功能特徵作爲分類標準的條件下, 可以根據語義特 徵來分類, 但是, 這樣分出的次類, 一定要能從功能方面得到驗證, 即每個次類都要有自己內 部大致一致的語法功能特徵。" p.57 


\begin{tabular}{|c|c|c|}
\hline & $\diamond \quad$ Doubtful & \\
\hline & Speaker's Attitude & \multirow[t]{2}{*}{ 玩吓手，或者 } \\
\hline & $\diamond \quad$ Surprise & \\
\hline & $\diamond \quad$ Determination & 點(不)知, 竟然 \\
\hline & $\diamond \quad$ Speaker's expectations & 一於，幾大 \\
\hline & $\diamond \quad$ Opposed speaker's expectations & 都係，情願 \\
\hline & $\diamond \quad$ Concession & 硬係，偏偏 \\
\hline & $\diamond \quad$ Strong emotions or emphasis & 話晒，好好醜醜 \\
\hline & $\diamond \quad$ Suggestion or persuasion & 直情，先(至) \\
\hline & $\diamond \quad$ Contrary fact & 千祈，何苦 \\
\hline & $\diamond \quad$ Understanding & 反爲，實則 \\
\hline & \multirow[t]{2}{*}{ Speaker's Viewpoint } & 原來，唔怪(之)得 \\
\hline & & 計正，聽講(話) \\
\hline Negation Adverb & "not" & 唔, 無 \\
\hline \multirow[t]{9}{*}{ Scope Adverb } & "All" are affected & 山蹦呤，通通 \\
\hline & "Part" are affected & 淨係，偏偏 \\
\hline & Upper limit & 頂多，極其量 \\
\hline & Lower limit & 起碼，座底 \\
\hline & Addition & 亦(都), 又 \\
\hline & Total number & 足足, 加加埋埋 \\
\hline & Approximate number & 約莫，大大話話 \\
\hline & "Almost" & 差啲, 幾乎 \\
\hline & Spatial scope & 隨地，到處 \\
\hline \multirow[t]{3}{*}{ Time Adverb } & Past/present/future time reference & 試過，第二時 \\
\hline & Frequency & 有啲，無幾何 \\
\hline & Duration & 枕長，轉下下眼 \\
\hline
\end{tabular}

Table 3: Subclassification of adverbs in Cantonese and their grammatical meanings

All the examples of these seven types of adverbs are given in Appendix 2.

Note that distinct senses of an adverb are represented by subscripted small letters, a, b, c, etc. Manner adverbs, among all, take up the largest proportion. This fact perhaps reflects that adverbs are mainly used for describing the manner in which an action is taken place. 


\section{Syntactic Functions of Adverbs}

\subsection{INTRODUCTION}

When a word class is defined, the main emphasis is given to its members: which word should be included and which should not. Even if it might be possible to list out all the members of a word class, it is not of great importance in the study of grammar, which aims at establishing rules for descriptively or prescriptively correct use of language. As Chao Yuen Ren (1968:497) points out, "while it is logically sufficient to define classes by enumeration, it may not seem satisfactory from the point of grammar, and we shall try to state whatever common property the class may have".

Another means of classifying words is by morphological characteristics; in English, it is not difficult to find that nearly most of the adverbs are morphologically marked by the derivational suffix -ly as in obviously (Kjellmer, 1984:1;

Burton-Roberts, 1991:165). Other adverbs with no such common property should be marked as exceptional. Nevertheless, Cantonese adverbs, like Mandarin Chinese counterparts, are not overtly marked by any morphological characteristics. Therefore, we should, again, find another means to determine the common property of adverbs. Chao suggests, "most words, however, belong to one part of speech or another by virtue of their positions in functional frames" (p.501), in addition to what he notes earlier that "the majority of Chinese words should have limited functions" (p.498). In other words, the common property of a class of words should be determined by their positions in a functional frame, or simply, by their functions. 
According to Chao Yuen Ren (1968:767), "adverbs are attributes or modifiers, of verbs, including adjectives, and of other adverbs, as in 先走 xiān zŏu 'go first', 最 美 zui měi 'most beautiful', 不一定 bùjiding 'not necessarily'". English adverbs more or less function in the same way. Quirk and Greenbaum (1973:125) states that "there are two types of syntactic function that characterise adverbs, but an adverb need have only one of these:
(1) Adverbial
(2) Modifier of adjective and adverb".

They do not exactly point out as Chao does that adverbs are modifiers of verbs. However, they further classify adverbial as mentioned in (1) above into three types, semantically. They are called adjuncts, disjuncts and conjuncts, which are named differently as circumstance adverbials, stance adverbials and linking adverbials, respectively, in the Longman Grammar of the Spoken and Written English (1999).

As Hoye (1997:24) points out, this classification of adverbs suggests that adverbs may function intrasententially (where they are relatively integrated within clause structure), sententially (where they are relatively peripheral to clause structure) or intersententially (where they have a linking function and conjoin clauses). Similarly, Jin Conggao (2000) divides adverbs into two major groups, word-modifying adverbs and sentence-modifying adverbs solely on the basis of their syntactic functions. These two studies both serve as a good testimony of the contention given by Greenbaum (1969:15) that "there is a gradient of integration of adverbs in the clause and not a sharp break between the integrated and the unintegrated".

More specifically, adjuncts or circumstance adverbials provide information about the action or state described in the clause by specifying time, place or manner. In other words, they modify the verb with respect to three domains, namely time, place and manner. Hence, although Quirk and Greenbaum do not clearly state that 
adverbs are modifiers of verbs, they do mention that adverbs syntactically function as adjuncts which "are integrated within the structure of the clause to at least some extent".

On the other hand, Chao's explanation on functions of adverbs is not without its own shortcoming. Some adverbs are not necessarily modifiers of verbs (Tao Hongyin, 2000), adjectives, and other adverbs. Chao divides adverbs into several sub-classes, among which, he proposes adverbs of evaluation and adverbs of contingency. He does not explain explicitly what these two sub-classes of adverbs mean semantically. However, as their names suggest and as inferred from the examples given, these adverbs provide information about speaker's judgment or evaluation on the situation described in the clause as in the former, and epistemic condition on the clause as in the latter. These two sub-classes of adverbs are not restricted to modify verbs or verb phrases but modify the clause as adverbial. It is therefore not sufficient to claim that adverbs function primarily as modifiers of verbs only (Schank, 1974:66; cf. Yang Defeng,1999).

To my knowledge, the Longman Grammar of the Spoken and Written English (1999) gives the simplest but insightful remarks on functions of adverbs that adverbs function as an element of the clause [as adverbial] or they are integrated into an element of the clause [as modifier]. By the same token, adverbs in Cantonese serve two major functions: firstly, by functioning in a phrase, an adverb may be a modifier of verb phrase, adjective phrase and noun phrase; secondly, by functioning in a clause, an adverb serves as an adverbial.

\subsection{FUnCTIONS IN A PHRASE}

\subsubsection{Modifier of Verb Phrase}

After careful examination of the corpus, it was found that manner, degree, scope and negation adverbs modify verb phrases. 
Manner adverbs modify verb phrases by being put as close to the verb phrases as possible. This fact is reflected by the observation that when the verb phrase consists of both an auxiliary verb and a main verb, the manner adverb tends to be placed after the auxiliary verb rather than before it, so as to be nearer to the verb phrase it modifies (Wong, 2001a; 2001b). As shown in the following examples, manner adverbs follow auxiliary verbs such as 會 wui 5 "could", 可以 ho2ji5 "can" and 想 soeng2 "wish to" and immediately precede main verbs.

(1) 我詅佢唔會特登撞我啩。 ngo5 nam2 keoi5 $m 4$ wui5 dak6dang1 zong6 ngo5 gwaa3

I think he not could intentionally hit me FP "I didn't think he intentionally hit me."

（2）即係諗住盡量唔好問呢, 㖇變咗啲人呢就可以快啲完結咽個。 zik1 hai6 nam2-zyu6 zeon6loeng6 m4hou2 man6 nel gam2 bin3-zo2 dil jan4 nel zau6 ho2ji5 faai3di1 jyun4git 3 go2 go3 that-is think-ASP as-much-as-possible do-not ask MP such-that turn-out-ASP PL people MP then can quickly finish that $\mathrm{M}$

"That is I've tried not to ask, then people can finish (the discussion) earlier."

(3) 我又唔想特登搬入䟿住喎。 ngo5 jau6 $m 4$ soeng2 dak6dang1 bun1 jap6lai4 zyu6 wo3

I but not want intentionally move come-in live FP

"But I don't want to move in for this purpose."

Without auxiliary verbs, manner adverbs come immediately before the whole verb phrase.

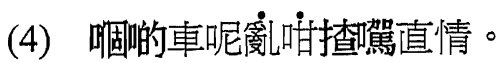

go2 dil cel nel lyun2gam3 zaal gaa3 zik6cing4

that PL car MP disorderly drive FP simply

"Those cars simply drove disorderly." 
(5) 又一樣, 哩件事照樣發生。

jau6 jat ljoeng6 nil gin6 si6 ziu3joeng2 faat3sang1

also same this $M$ thing as-usual happen

"The same thing happened."

(6) 點解佢猛咁撞呢?

dim2gaai2 keoi5 maang5gam 3 zong6 ne1

why s/he desperately hit FP

"Why did he hit you desperately?"

Degree adverbs and scope adverbs, like manner adverbs, are placed before the verb phrase. It is interesting to point out that in example (7) the degree adverb 好 hou2 "very" is modifying the verb phrase made up of a psychological verb 怕 paa3 "fear". ${ }^{18}$ English degree adverbs, on the contrary, do not modify psychological verbs as in $*_{\text {very }}$ like $e^{19}$ as well as *very detest ${ }^{20}$. This is one of the major differences between English and Chinese adverbs on modifying verb phrases.

(7) 我好怕喵固啲嘢。

ngo5 hou 2 paa3 go2 dil jes

I very fear that PL thing

"I am scared of that thing."

(8) 完全無晒規律啊, 我覺得, 一放假。

jyun4cyun4 mou5-saai3 kwailleot6 aa3 ngo5 gok3dakl jat 1 fong3gaa3 totally have-not-COMP order MP I think once have-holiday

"Totally without any order, I think, once it's holiday."

\footnotetext{
${ }^{18}$ See Yuan Mingjun (1999) for the types of verbs (other than psychological verbs) being modified by degree adverbs in Mandarin Chinese.

${ }^{19}$ C.f. Cantonese 好鍾意 hou2 zunglji3 "like (it) very much"; Mandarin 很喜歡 hĕn xihuān "like (it) very much"

${ }^{20}$ C.f. Cantonese 好憎 hou2zang1 "hate (it) very much"; Mandarin 很厠惡 hĕn yànwù "hate (it) very much"
} 
(9) 佢淨係話呢, 如果唔搭內陸機仲好喇。

keoi5 zing6hai6 waa6 nel jyu4gwo2 m4 daap6 noi6luk6keil zung6 hou2 laal $\mathrm{s} /$ he only say MP if not ride inland-plane even good FP

"He only said that it was even better not to take the inland-plane."

(10) 但係亦都有噉嘅可能佢瞓着咗呢。

daan6hai6 jik6dou1 jau5 gam2 ge3 ho2nang4 keoi5 fan3zoek6-zo2 ne1

but also have like-that GE possibility s/he fall-asleep-ASP FP

"But there was a possibility that he had fallen asleep."

When negation adverbs modify verb phrases, their positioning is largely dependent on the scope of negation. If they come before the auxiliary verb (shadowed), they negate both the auxiliary verb and the main verb. As in the examples below, negation adverbs negate the possibility of doing an action.

(11) 跟住個導遊就話任哋答里島政府呢就規定呚咱啲樓呢, 唔可以起得高過峇里島最 高㧽棵椰子樹囖。 gan1zyu6 go3 dou6jau4 zau6 waa6 keoi5dei6 Baallei5dou2-zing3fu2 nel zau6 kwailding6-zo2 go2 dil lau2 nel $\mathbf{m} 4$ ho2ji5 hei2-dakl goulgwo3 Baallei5dou2 zeoi3 goul go2 pol je4zilsyu6 lol then M tour-guide then say their Bali-government MP EMPH stipulate-ASP that PL building MP not can build-COMP higher Bali most high that M coconut-tree FP "Then, the tour guide said it was stipulated by the Bali government that no buildings were built higher than the highest coconut tree in Bali."

(12) 我諗佢唔會特登撞我啩。 ngo5 nam2 keoi5 $\mathbf{m} 4$ wuit dak6dang1 zong6 ngo5 gwaa3

I think s/he not could intentionally hit me FP "I didn't think he intentionally hit me."

On the other hand, when they come after the auxiliary verb, they negate only the main verb. If we move the negation adverbs from their original position to a new one i.e. after the auxiliary verb, the sentences would mean differently: they express the possibility of not doing an action, like the following fabricated examples with the negation adverb being transposed from its original location: 
（13）跟住個導遊就話佢哋答里島政府呢就規定咗喵啲樓呢, 可以唔起得高過答里島最 高㧽棵椰子樹簕。

ganlzyu6 go3 dou6jau4 zau6 waa6 keoi5dei6 Baallei5dou2-zing3fu2 nel zau6

kwailding6-zo2 go2 dil lau2 nel ho2ji5 m4 hei2-dakl goulgwo3 Baallei5dou2 zeoi3

goul go2 po1 je4zilsyu6 lo1

then M tour-guide then say their Bali-government MP EMPH stipulate-ASP that PL

building MP can not build-COMP higher Bali most high that M coconut-tree FP

"Then, the tour guide said it was stipulated by the Bali government that buildings could be built not higher than the highest coconut tree in Bali."

(14) 我諗佢會唔特登撞我啩。

ngo5 nam2 keoi5 wui5 $\mathbf{m} 4$ dak6dang1 zong6 ngo5 gwaa3

I think s/he could not intentionally hit me FP

"I thought he could not intentionally hit me."

Without auxiliary verbs, negation adverbs are normally put before verb phrases in modifying them.

(15) 因爲佢啯陣時呢, 我哋報名嘅時候, 佢無講俾我哋聽嗎。 jan1wai6 keoi5 go2zan6si4 nel ngo5dei6 bou3meng2 ge3 si4hau6 keoi5 mou5 gong2 bei2 ngo5dei6 teng1 gaa3

because it by-that-time MP we enroll GE time s/he have-not tell to us listen FP

"By the time we enrolled in the tour, he didn't tell us about it."

(16) 不過我本時無也用嗎喎。

bat1gwo3 ngo5 ping4si4 mou5mat1 jung6 gaa 3 wo 3

but I normally almost-not use FP FP

"But I don't use (it) very often."

\subsubsection{Modifier of Adjective Phrase}

In English, adverbs can either premodify or postmodify adjectives, as illustrated in the following examples. 
(17) He is a very interesting person.

(18) It is a really beautiful place.

(19) The box is not large enough.

(20) This seems long ago.

Similarly, in Cantonese, degree adverbs and negation adverbs are premodifiers of adjective phrases and some scope adverbs, for example, 咁滯 gam3zai6 "almost" and 七滯 mat1zai6 "almost" postmodifiers.

(21) 噉喵餐就幾好囖我覺得。

gam2 go2 caan1 zau6 gei2 hou2 lo1 ngo5 gok3dak1

IP that M EMPH quite good FP I think

"I thought that meal was quite good."

(22) 係咪因爲架車郁得太緊要啊?

hai6-mai6 janlwai6 gaa3 cel jukl-dakl taai3 gan2jiu3 aa3

be-then because $\mathrm{M}$ car shake-COMP too serious FP

"Is it the case that the bus had been shaken too seriously?"

(23) 㖣靚㧽啲唔會要嗎。

m4 leng3 go2 dil $m 4$ wuis jiu 3 gaa3

not pretty that PL not will need FP

"I won't buy those which are not good."

(24) 佢無話咁絕情嘅。

keoi5 mou5waa6 gam3 zyut6cing4 ge3

$\mathrm{s} /$ he never so cruel FP

"He is never so cruel."

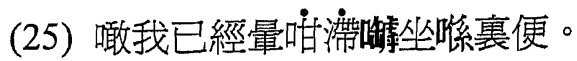

gam2 ngo5 ji5ging1 wan4 gam3zai6 laa3 co5 hai2 leoisbin6

IP I already faint almost FP sit at inside

"I almost fainted inside the bus." 
(26) 啲橙好大個, 文甜。

dil caang2 hou2 daai6 go3 jau6 tim4

PL orange very big $\mathrm{M}$ also sweet

"The oranges are very big and they taste good."

\subsubsection{Apparent Modifier of Prepositional Phrase}

English adverbs premodify prepositions or prepositional phrases as in "The nail went right through the wall." given by Quirk and Greenbaum (1973:127). Chao Yuen Ren does not comment on the capability of Chinese adverbs as modifiers of prepositions or prepositional phrases. Ding Shengshu et al. (1979:7), however, point out that adverbs can be modifiers of prepositions in Mandarin Chinese.

(27) 只把這個問題提出來。

zhíbăzhège wèntítí chülai

only PREP this-M question put-forward come-out

"Only this question is put forward (for discussion)."

However, this example should not be regarded as an illustration of an adverb modifying a preposition or prepositional phrase. Semantically speaking, the adverb 只 zhímodifies the object of preposition, 這個問題 zhège wèntí"this question". In other words, the adverb is semantically modifying the object of preposition, which is a noun phrase, but not the preposition or prepositional phrase. On the other hand, syntactically speaking, the adverb is modifying both the prepositional phrase and the following verb. To put it another way, the adverb is not modifying the prepositional phrase alone. Hence, from both the semantic and syntactic perspectives, an adverb in Mandarin does not serve as a modifier of prepositional phrase.

Following on from this, the fact that an adverb comes immediately before a preposition or prepositional phrase does not necessarily imply that the former modifies the latter, as illustrated in the examples (28) and (29). In example (28), the degree adverb 好 hou 2 "very" is put before the prepositional phrase, highlighted with shadowing here, but it is actually modifying the adjective phrase 唔公平 $m 4$ 
gunglping4. Similarly, in example (29), the same degree adverb 好 is modifying the adjective 驚 gengl "frightened" that follows the prepositional phrase 星佢 dang6 keoi5. The prepositional phrases in both sentences give us information about the target that the speakers want to comment on.

(28) 你唔覺得好對咽固個廠商唔公平嘅咩?

nei5 $\mathrm{m} 4$ gok3dakl hou 2 deoi3 go2 go3 cong2soeng1 m4 gunglping4 ge3 me1

you not think very to that M company not fair FP FP

"Do you think it's really very unfair to the company?"

(29) 真係好㦹佢驚嗎。

zan1hai6 hou2 dang6 keoi5 geng1 gaa3

really very for him/her fear FP

"I feel really very worried for him."

Other examples include adverbs modifying verb phrases that, again, follow prepositional phrases expressing the target, place and agent of action.

(30) 夾硬將自己呢隻腳呢紮住佢。

gaap3ngaang2 zoeng1 zi6gei2 ne1 zek3 goek3 nel zaak3-zyu6 keoi5

grudgingly PREP oneself MP M feet MP tie-COMP it

"She just grudgingly tied up her feet."

(31) 喏啱同我調轉。

ngaam1ngaam1 tung4 ngo5 diu6zyun3

exactly to me opposite

"(Yours) is exactly opposite to mine."

(32) 矮佢實無係 Body Shop 幫我買嘢喇。

o4 keoi5 sat6 mou5 hai2 body shop bong1 ngo5 maai5 je5 laal

IP s/he certainly have-not at body shop help me buy thing FP

"He certainly hadn't helped me buy something from Body Shop." 
(33) 但係佢無俾人哋柴臺咩?

daan6hai6 keoi5 mou5 bei2 jan4dei6 caai4toi4 mel

but s/he have-not by people boo-off FP

"But had he been booed off by others?"

\subsubsection{Modifier of Noun Phrase}

It has often been stated in the literature that English adverbs do not modify noun phrases; however, some of the adverbs that are premodifiers of an adjective display an orientation to a co-occurring noun subject (Valera, 1998). As in the following examples taken from Valera's study (1998:264), adverbs that are capable to acquire an implicit intensive relationship with the subject of a sentence are said to be subject-oriented.

(34) In the old days, she had been harsh and stiff; afraid of her husband and yet arrogantly proud that she had a husband strong and fierce enough to make her afraid.

(35) His women are excitingly depraved, but they aren't sick, they are anything but sick; they convey a terrific sense of well-being.

This subject-orientation of adverbials transfers the primary reference or characterisation of the adverbial from the adjective to the accompanying subject. Syntactically, the adverbs are still modifying the adjectives.

In Mandarin Chinese, Chao Yuen Ren (1968:767) is arguably the first to point out that adverbs can modify nouns to a certain extent: "[a]n adverb does not normally modify nouns. When it does, it really modifies the noun or the nominal expression as a predicate or, more questionably, as a subject". Until recently, Zhang Yisheng's (2000:153-179) study on this issue offers the most perceptive insights into the semantic properties of nouns that enable them to be modified by adverbs. His study, to the best of my knowledge, is the most comprehensive description of adverbs modifying nouns. 
At first sight, Cantonese adverbs are like Mandarin Chinese ones in that they can be used to modify noun phrases as predicate and subject.

(36) 而家先(至)一點鐘。(predicate) ji4gaal sin1(zi3) jat1 dim2zungl now just one o'clock "It's just one o'clock now."

(37) 你直情俊瓜喇! (predicate) nei5 zik6cing4 so4gwaal laal you simply a-fool FP "You are simply a fool!"

(38) 但係個個人都會有啲關於去旅行嘅時候呢, 咽啲鬼故駕。(subject) daan6hai6 go3go3 jan4 dou1 wui5 jau5 dil gwaanljyul heoi3 leoi5hang4 ge3 si4hau6 ne1 go2 dil gwai2gu2 gaa3 but every people all could have PL about go travelling GE time MP that PL ghost-story FP

"But everyone could have got some ghost stories about their own travelling."

(39) 係噉問埋哂啲呢懶係呢即係淨係佢做咽啲先諳叫固啲嘢呢。(subject) hai6gam2 man6-maai4-saai3 di1 nel laan2 hai6 nel zikl hai6 zing6hai6 keoi5 zou6 go2 dil sin1 sik1 go2 dil je5 ne1 continuously ask-COMP-COMP PL MP pretended be MP that-is only s/he do that PL then know that PL stuff FP "They just kept asking stuff that seemed to be understood only by people in the industry."

Nevertheless, what is striking about Cantonese adverbs and makes it different from Mandarin Chinese adverbs is that they are not restricted to modify noun phrases functioning as predicate and subject only. They are relatively free in modifying noun phrases as object, as illustrated using scope adverbs in the following. 
(40) 但係哩個嚴重嘅情況即係唔係淨係今年個喎。(object) daan6hai6 nil go3 jim4zung6 ge3 cing4fong3 zik1 hai6 m4 hai6 zing6hai6 gam Inin2 go3 wo3

but this M serious GE situation that-is not be only this-year FP FP

"But this situation happens not only this year."

(41) 我由個 lobby 呢行到去我間房要成十幾分鐘茄。(object) ngo5 jau4 go3 lobby nel haang4-dou3 heoi3 ngo5 gaanl fong2 jiu3 seng4 sap6gei2 fanlzungl ge2

I from M lobby MP walk-COMP to my M room have approximately ten-something minutes FP

"It took almost more than ten minutes to walk back to my room from the lobby."

（42）跟住計起上嚟年薪過三十萬咁滯喇差唔多。(object)

gan1zyu6 gai3-hei2soeng5lai4 nin4san1 gwo3 saam1sap6 maan6 gam3zai6 laa1 caalm4dol then calculate-COMP yearly-salary over thirty ten-thousand almost FP almost "The annual salary is almost over three hundred thousands."

In particular, degree adverbs are remarkably free in modifying noun phrases in both Cantonese and Mandarin Chinese. Many Chinese linguists have noticed that "Adverb of Degree + Noun" structure is growing more and more popular in both spoken and written language, and studied the syntactic, semantic and pragmatic aspects of this construction (Chu Zexiang and Liu Jiesheng, 1997; Xing Fuyi, 1997; Wang Xiaoxin and Zhang Ge, 1998; Lei Jiezhen, 1999; Xia Huimin, 1999; Ren, Zhijing, 2001). However, the use of degree adverbs in modifying noun phrases in Cantonese does not exhibit originality nor novelty as that in Mandarin Chinese. This is a commonplace in Cantonese conversational data. Some examples are given as follows.

(43) 都係一啲好生活嘅一啲笑料嚟嗎。 dou1 hai6 jat1dil hou2 sang1wut6 ge3 jat1di1 siu3liu2 lai4 gaa3

all be one PL very daily-life GE one PL gag come FP

"They are all gags which are closely related to our daily life." 
(44) 可能呢都會係講一啲比較基層嘅市民。

ho2nang4 nel doul wui5 hai6 gong2 jat1 dil bei2gaau3 geilcang4 ge3 ci5man4

perhaps MP after-all could be say one M relatively grass-roots GE citizens

"Perhaps, much has been said about people of relatively lower social class, the grass roots."

(45) 我覺得佢個樣好生意人啊。

ngo5 gok3dak1 keoi5 go3 joeng2 hou2 saanglji3jan4 aa3

I feel his $\mathrm{M}$ appearance very businessman FP

"I think his appearance exactly looks like a businessman."

（46）係啊, 但係我今日我覺得諗住著白色又未到夏天喎, 著咁夏天做也嘢叮?

hai6 aa3 daan6hai6 ngo5 gamljat6 ngo5 gok3dak1 nam2-zyu6 zoek3 baak6sik1 jau6 mei6 dou 3 haa6tin1 wo3 zoek3 gam3 haa6tin1 zou6 matlje5 aa1

be FP but I today I feel think-ASP wear white-colour but not-yet come summer FP wear so summer do what FP

"Yes, actually today I am thinking about wearing white clothes but the summer has not come so why should I wear white colour, so summer-like?"

\subsection{FUNCTION IN A CLAUSE - AdVERBIAL}

On modifying clauses or sentences, time adverbs, modal adverbs and conjunctive adverbs are concerned. As Hoye (1997:145) points out, most grammars distinguish between adverbs which modify the sentences in which they occur, referred to as sentence-modifying adverbs, and those adverbs which modify a word or word-group within the sentence, traditionally known as word or word-group modifiers. It is clearer that modal adverbs and conjunctive adverbs modify on the sentence level than that time adverbs do as the former tend to be less closely related to the verb than the latter. Hence, it is not clear how the distinction between sentence modifiers and word-group modifiers should be made, as far as time adverbs are concerned.

Generally speaking, time adverbs specify the time, frequency and duration of, not only an action, but also the entire event. In particular, frequency time adverbs 
specify how often the whole event recurs. We thus tentatively conclude that time adverbs are sentence adverbs and function in a sentence.

（47）我咽陣時呢，試過入去一間鋪頭呢，賣啲帽嗎。 ngo5 go2zan6si4 ne1 si3gwo3 jap6heoi3 jat1 gaan1 pou3tau2 ne1 maai6 di1 mou2 gaa3

I at-that-time MP once enter one M shop MP sell PL hat FP

"I once went to a shop selling hats."

(48) 佢不舅都唔係係廠度做文員。

keoi5 bat1nau1 dou1 $m 4$ hai6 hai2 cong2-dou6 zou6 man4jyun4 s/he all-along EMPH not be at factory-LOC do clerk

"She wasn't working as a clerk at the factory at all."

Modal adverbs are mainly used to express speaker's attitude, certainty/doubt and point of view of the situation described in the clause. As they modify the whole sentence, they are not restricted to be placed as close to any sentence constituent as manner and degree adverbs are. They are mobile in their positioning in the sense that they can occur before the subject, after the subject, before the sentence boundary or after the sentence boundary (as marked by the final particles).

(49) 唔通你特登去問佢咩?

m4tung1 nei5 dak6dang1 heoi3 man6 keoi5 me1

rhetorical-question you deliberately go ask him/her FP

"You can't just ask her directly!"

(50) 唔係肥倫, 肥倫我實認得喇。

$m 4$ hai 5 Fei4leon4 Fei4leon4 ngo5 sat6 jing6-dak1 laal not be Fat-Lun Fat-Lun I definitely recognise-COMP FP

"IT's not Fat Lun, Fat Lun I could definitely recognise."

（51）無也去過也嘢地方其實。 mou5mat1 heoi3-gwo3 matlje5 dei6fong1 kei4sat6 almost-not go-ASP what place actually

"Actually, not many places had we visited." 
(52) 喵啲車呢亂咁揸嗎㨁情。

go2 dil ce1 nel lyun2gam3 zaal gaa3 zik6cing4

that PL car MP disorderly drive FP simply

"Those cars simply drove disorderly."

Conjunctive adverbs modify on the clause level as they typically connect two clauses.

(53) 因爲呢佢開咗冷氣就闍晒啲窗。

jan1wai6 nel keoi5 hoil-zo2 laang5hei3 zau6 saan1-saai3 dil coeng1 because MP it open-ASP air-conditioning then close-COMP PL window "Because all the windows were closed when air-conditioning was on."

(54) 噉去到呢, 佢就落車。

gam2 heoi3dou3 nel keoi5 zau6 lok6ce1

IP arrived MP s/he then get-off

"He got off when he arrived."

(55) 搬入嚟架車咪擺-擺啋哩邊囖。

bun1 jap6lai4 gaa3 cel mai6 baai2 baai2 hai2 nil binl lo1

move come-in $\mathrm{M}$ car then put put at this place FP

"If I move in, the car can be parked there."

\subsection{SUBSTANTIATION OF SUB-CLASSIFICATION OF ADVERBS}

As noted earlier, it is not objective enough to rely on meanings in sub-classification. It would be better if we can corroborate the sub-classification syntactically. In the present chapter, it was found that different sub-classes modify different elements, as illustrated in Table (4).

In this table, adverbs fall into two major divisions, one division in which its members function in a clause and the other division function in a phrase. The 
former category differentiates between three adverb sub-classes, namely conjunctive adverbs, modal adverbs and time adverbs, according to their grammatical meanings. The latter category is taken up by the other adverb sub-classes, including scope adverbs, degree adverbs, manner adverbs and negation adverbs. They are distinguished from one another in modifying different elements syntactically.

\begin{tabular}{|l|c|c|c|c|}
\hline Adverb sub-classes & $\begin{array}{c}\text { Modifier of } \\
\text { Verb Phrase }\end{array}$ & $\begin{array}{c}\text { Modifier of } \\
\text { Adjective Phrase }\end{array}$ & $\begin{array}{c}\text { Modifier of } \\
\text { Noun Phrase }\end{array}$ & $\begin{array}{c}\text { Modifier of a } \\
\text { Clause }\end{array}$ \\
\hline Conjunctive Adverb & & & & $\checkmark$ \\
\hline Modal Adverb & & & & $\checkmark$ \\
\hline Time Adverb & & & & $\checkmark$ \\
\hline Scope Adverb & $\checkmark$ & $\checkmark$ & $\checkmark$ & \\
\hline Degree Adverb & $\checkmark$ & $\checkmark$ & $\checkmark$ & \\
\hline Manner Adverb & $\checkmark$ & & & \\
\hline Negation Adverb & $\checkmark$ & $\checkmark$ & & \\
\hline
\end{tabular}

Table 4: An illustration of different adverb sub-classes modifying different elements

Schematically, adverbs are subcatgorised on both syntactic and semantic grounds as follows:

\section{Classified by SYNTACTIC Classified by GRAMMATICAL FUNCTIONS: MEANINGS}

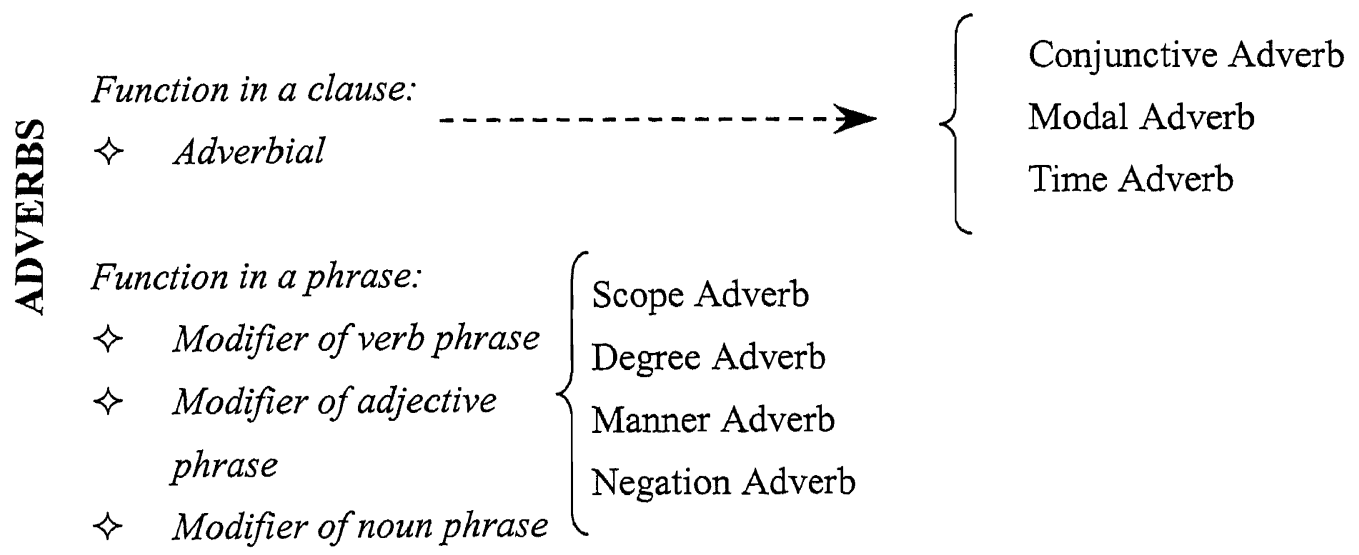

Figure 3: Syntactic and semantic subclassification of adverbs in Cantonese 


\subsection{SUMMARY}

Cantonese adverbs can be modifiers of verb phrase, adjective phrase and noun phrase. Not every adverb is used in modifying these phrases. Manner, degree, scope and negation adverbs modify verb phrases. They are put as close to the verb phrases as possible, except negation adverbs whose positioning is governed by the scope of negation. Only degree, negation and scope adverbs are found to modify adjective phrases. Furthermore, degree adverbs and scope adverbs are used in modifying noun phrases. Adverbs do not modify prepositional phrases; rather, they modify the verb/adjective phrases that follow the prepositional phrases. Apart from functioning in a phrase as modifier, some adverbs can modify a clause or sentence as adverbial, namely time adverbs, modal adverbs and conjunctive adverbs.

\section{A Contrastive Analysis of Cantonese, English and Mandarin Chinese on the} Syntactic Functions of Adverbs:

In the present study, Cantonese can be distinguished from English and Mandarin Chinese in the following ways (Wong, 2002).

Cantonese as distinguished from English:

(1) English degree adverbs cannot modify psychological verbs but both Cantonese and Mandarin Chinese ones can do so.

(2) English degree adverbs cannot function as modifier of noun phrase, in contrast to Cantonese and Mandarin Chinese ones.

(3) English adverbs can premodify prepositions and prepositional phrases but both Cantonese and Mandarin Chinese ones cannot.

Cantonese as distinguished from Mandarin Chinese:

(4) As modifiers of noun phrases, Cantonese adverbs exhibit their own peculiarities in that (i) scope adverbs can modify noun phrases as object, unlike their Mandarin counterparts; (ii) degree adverbs modifying nominal expressions is a commonplace in Cantonese whereas this is regarded as novelty in both spoken and written texts in Mandarin Chinese. 


\section{Conclusion and Further Research}

This chapter concludes with the major findings of the present study. It also presents some suggestions on possible further research that could be conducted on Cantonese adverbs.

\subsection{CONCLUSIONS}

The present study gives a fairly comprehensive list of 544 Cantonese adverbs, as exemplified in Appendix 1. More than half of the adverbs are disyllabic, which are overwhelmingly cognates of Mandarin. By contrast, Cantonese-specific adverbs are predominantly trisyllabic and polysyllabic, suggesting that polysyllabicity is one of the characteristics of Cantonese-specific adverbs. Furthermore, at least 17 recurrent patternings are found to be typically associated with disyllabic, trisyllabic and polysyllabic adverbs, and reduplication, among these systematic patterns, is another characteristics of Cantonese-specific adverbs.

All the adverbs collected in this study are further classified into seven types in Appendix 2 to reflect their shared grammatical meanings such as manner, time, scope, etc. Not only are these adverb sub-classes distinct from one another semantically, they differ syntactically in modifying different elements in the clause. These syntactic properties evidently give support to the semantic classification of adverbs proposed in the present study. 
Apart from offering some observations on the morphology and semantics of Cantonese adverbs, this study also examines their syntactic characteristics. Traditionally, an adverb is treated as a modifier of the verb/adjective. Chao Yuen Ren (1968:767) insightfully adds that an adverb can modify a noun phrase which functions as a predicate or subject of the sentence. Cantonese adverbs, as previously mentioned, do exhibit all these syntactic properties.

More importantly, adverbs in Cantonese are distinguished from their counterparts in English and Mandarin Chinese in modifying noun phrases. In contrast to English degree adverbs which do not normally modify a noun phrase, Cantonese degree adverbs do. Furthermore, Cantonese adverbs of scope modify noun phrases functioning as object in a clause, as opposed to those in Mandarin Chinese.

\subsection{AREAS OF FUTURE RESEARCH}

One thing is clear from the review of the observations that the current study made - Cantonese adverbs exhibit peculiar properties in different aspects of grammar. In this section, we would like to suggest research area where other interesting characteristics of adverbs are possibly found.

\subsubsection{Productivity of Adverbs}

There are about 50 monosyllabic adverbs found in the present study. These adverbs differ from one another in their productivity in that some of them are used with more verbs/adjectives, that is to say, more productive, than the others. These productive adverbs are 白 baak6 "in vain", 重 cung4 "again", 亂 lyun2 "thoughtlessly"，最 zeoi3 "the most"，專 zyun1 "specially"，惡 ngok3 "with difficulty", 實 sat6 "certainly",盡 zeon6 "exhaustively", etc. Other adverbs are less productive, for example, 初 col "at first", 新 sanl "newly", etc. Even less productive ones are mostly adverbs adopted from the Mandarin Chinese such as 牛 
bun3 "half", 乾 gon 1 "in a dry manner", 力 lik6 "make every effort", 漫 maan6 "casually", 誤 $m 6$ "mistakenly", 互 wu6 "each other", 直 zik6 "straight; directly", etc. We can look more closely at the productivity of these monosyllabic adverbs in further research. We can also consider the productivity of disyllabic and polysyllabic adverbs likewise.

\subsubsection{Distribution of Adverbs (with respect to sentence types)}

Modal adverbs tend to differ with respect to their occurrence in different sentence types (see, for example, Duan Yehui, 1999). Some modal adverbs, for instance, 唔通 $m 4$ tungl "can it be that", 究竟 gau3ging2 "actually" and 到底 dou3dai2 "actually" are usually used in interrogative sentences. Other adverbs such as 夠 gau3 "too" and 簡直 gaan2zik6 "simply" are predominantly used in exclamatory sentences and adverbs like 何必 ho4bit1 "why bother", 何苦 ho4fu2 "why bother", 有幾何 jau5gei2ho2 "seldom" and 唔通 m4tung1 "expressing rhetorical question" in rhetorical questions. Furthermore, some adverbs must be used in negation sentences, namely 好日 hou2jat6 "rarely; seldom", 七滯 mat1zai6 "not quite", 未賞 mei6soeng4 "not" and 無日 mou5jat6 "all the time". The distribution of adverbs in different types of sentences could be subject to further research in order to gain better insight into the pragmatic functions of these adverbs.

\subsection{FINAL REMARKS}

The present study has shed new light on the morphological, semantic and syntactic characteristics of Cantonese adverbs. Most importantly, the identification of adverbs in Cantonese will perhaps make the classification system of words more comprehensive and ultimately, make the process of the part-of-speech tagging easier. 


\section{Appendix 1: A List of Cantonese Adverbs}

\begin{tabular}{|c|c|c|c|c|c|}
\hline No. & \begin{tabular}{|l} 
Cantonese \\
Adverbs
\end{tabular} & Romanisation & Type $^{\mathrm{a}}$ & Meanings & Example Sentences \\
\hline 1 & 擺明 & baai2ming 4 & $\mathrm{CS}$ & "clearly" & 你擺明玩我啫? \\
\hline 2 & 百般 & baak3bunl & $\mathrm{CM}$ & "by every means" & 佢簡直係百般了難我喇。 \\
\hline 3 & 白 & baak6 & $\mathrm{CM}$ & "in vain; for nothing" & 呢次真係白做。 \\
\hline 4 & 白白 & baak6baak6 & $\mathrm{CM}$ & $\begin{array}{l}\text { "in vain; to no } \\
\text { purpose" }\end{array}$ & 我今次真係白白嘥晒啲錢。 \\
\hline 5 & 樹猿聲 & baang4baang2seng1 & CS & $\begin{array}{l}\text { "very quickly; at } \\
\text { once" }\end{array}$ & 仲唔嘭囍聲出去搵佢? \\
\hline 6 & 包 & baaul & CS & "certainly" & 今日包佢落雨。 \\
\hline 7 & 弊在 & bai6zoi6 & CS & "unfortunately" & 佢揫在唔嚟得呢。 \\
\hline 8 & 不特止 & bat1dak6zi2 & CS & "not only" & 佢逃學不特止, 仲偷眐㤭。 \\
\hline 9 & 不斷 & bat Idyun6 & $\mathrm{CM}$ & "continuously" & 佢不斷岁頭。 \\
\hline 10 & 不妨 & batIfong4 & $\mathrm{CM}$ & $\begin{array}{l}\text { "there is no harm in; } \\
\text { might as well" }\end{array}$ & $\begin{array}{l}\text { 唔開心不妨搵個朋友出洯傾 } \\
\text { 吓。 }\end{array}$ \\
\hline 11 & 不如 & bat1jyu4 & $\mathrm{CM}$ & $\begin{array}{l}\text { "it would be better } \\
\text { to" }\end{array}$ & $\begin{array}{l}\text { 啲畘都做完晒, 不如早帕走 } \\
\text { 好過。 }\end{array}$ \\
\hline 12 & 不期然 & bat1kei4jin4 & $\mathrm{CS}$ & "unexpectedly" & 我不期然諗起一件事。 \\
\hline 13 & 不鬼 & bat $1 \mathrm{kwai} 5$ & $\mathrm{CM}$ & " be worthy of " & 你不愧係佢個好朋友。 \\
\hline 14 & 不舅 & bat1laul & CS & $\begin{array}{l}\text { "consistently; all } \\
\text { along" }\end{array}$ & 我不舅都係住㗱香港䅂。 \\
\hline 15 & 不知 & batlzil & CS & $\begin{array}{l}\text { " to speaker's/hearer's } \\
\text { surprise" }\end{array}$ & 霆鋒個演唱會不知幾好啊。 \\
\hline 16 & 比較(上) & bei2gaau3(soeng6) & $\mathrm{CM}$ & "fairly; rather" & 哩種病比較難糹尾。 \\
\hline 17 & 本來 & bun2loi4 & $\mathrm{CM}$ & "originally" & 佢本來係個好人。 \\
\hline 18 & 牛 & bun3 & $\mathrm{CM}$ & "half" & 牛生熟；牛新舊 \\
\hline 19 & 差啲 & caaldil & CS & "almost" & 我差啲認唔到佢。 \\
\hline 20 & 查實 & caa4sat6 & CS & "in fact; actually" & 查實，使唔使噉做呢？ \\
\hline 21 & 齊齊 & cai4cai4 & CS & "together" & 我哋齊齊去玩。 \\
\hline 22 & 親口 & can1hau2 & $\mathrm{CM}$ & "(speak) personally" & 佢親口同我講嘅。 \\
\hline 23 & 親耳 & canlji5 & $\mathrm{CM}$ & "(hear) personally" & 我親耳㯖到鶷。 \\
\hline 24 & 親眼 & canIngaan5 & $\mathrm{CM}$ & "(see) personally" & 我親眼睇至到嘫噃。 \\
\hline 25 & 親手 & can 1 sau 2 & $\mathrm{CM}$ & $\begin{array}{l}\text { "with one's own } \\
\text { hands" }\end{array}$ & 你親手做㗎? \\
\hline 26 & 親自 & canlzi6 & $\mathrm{CM}$ & "in person" & $\begin{array}{l}\text { 麻煩你親自嚟攞番張信用咭 } \\
\text { 吅。 }\end{array}$ \\
\hline 27 & 趁早 & can $3 z o u 2$ & $\mathrm{CM}$ & "as early as possible" & 你趁早報名咪唔驚無位䈬。 \\
\hline 28 & 曾經 & cang4ging1 & $\mathrm{CM}$ & "ever" & 佢曾經坐過監。 \\
\hline 29 & 脆脆哋 & ceoi3ceoi2dei2 & CS & $\begin{array}{l}\text { "reluctantly; } \\
\text { grudgingly" }\end{array}$ & $\begin{array}{l}\text { 你咪脆脆哋收咗份禮物任 } \\
\text { 囖。 }\end{array}$ \\
\hline
\end{tabular}




\begin{tabular}{|c|c|c|c|c|c|}
\hline 30 & 隨處 & ceoi4cyu 3 & CM & "everywhere" & 你唔好將啲嘢了隨處掉喇！ \\
\hline 31 & 隨地 & ceoi4dei6 & $\mathrm{CM}$ & "anywhere" & 佢隨地拓㩊㩑。 \\
\hline 32 & 隨口 & ceoi4hau2 & $\mathrm{CM}$ & $\begin{array}{l}\text { "(speak) } \\
\text { thoughtlessly" }\end{array}$ & 你咪隨口噏喎！ \\
\hline 33 & 隨手 & ceoi4sau2 & $\mathrm{CM}$ & "conveniently" & 佢隨手攞起把遮就走咗。 \\
\hline 34 & 隨時 & ceoi4si4 & $\mathrm{CM}$ & "at all times" & 老人家隨時有病痛。 \\
\hline 35 & 始終 & ci2zungl & $\mathrm{CM}$ & "after all" & 佢始終都無嚟。 \\
\hline 36 & 次次 & $c i 3 c i 3$ & CS & "every time" & $\begin{array}{l}\text { 次次都遲成個鐘，係人都㷫 } \\
\text { 喇。 }\end{array}$ \\
\hline 37 & 遲啲(再) & ci4dil(zoi3) & CS & "later" & 我遲啲(再)還番啲錢俾你。 \\
\hline 38 & 遲早 & ci4zou2 & $\mathrm{CM}$ & $\begin{array}{l}\text { "some time in the } \\
\text { future; some day" }\end{array}$ & 間公司噉搞法,遲早要執笠。 \\
\hline 39 & 似乎 & cisfu4 & $\mathrm{CM}$ & "seemingly" & 佢似乎唔信喎。 \\
\hline 40 & 千新 & cin1kei4 & $\mathrm{CS}$ & "for heaven's sake" & 千祈唔好講俾人知。 \\
\hline 41 & 前後 & $\operatorname{cin} 4$ hau6 & $\mathrm{CM}$ & "altogether" & 我前後去咗三次。 \\
\hline 42 & 情願 & cing4jyun2 & $\mathrm{CM}$ & "would rather" & 我情願行路都唔願坐車。 \\
\hline 43 & 超 & $\operatorname{ciul}$ & CS & "super" & 佢行得超快啊。 \\
\hline 44 & 初 & col & $\mathrm{CM}$ & "at first" & 初學；初嚟 \\
\hline 45 & 初初 & colcol & $\mathrm{CS}$ & "at first" & 佢初初唔制, 卒之應承咗。 \\
\hline 46 & 初頭 & coltau4 & CS & "at the beginning" & 我初頭諗極都諗唔到點做。 \\
\hline 47 & 長 & coeng 4 & CS & "for a long time" & 隻新股可以短炒, 不宜長揸。 \\
\hline 48 & 速速 & cuklcukl & CS & $\begin{array}{l}\text { "very quickly; at } \\
\text { once" }\end{array}$ & 發生車禍就速速報警嘘。 \\
\hline 49 & 重 & cung4 & $\mathrm{CM}$ & "again; once more" & 你份功課要重做呀? \\
\hline 50 & 從來 & cung4loi4 & $\mathrm{CM}$ & "always; at all times" & $\begin{array}{l}\text { 哩個人好生面口，從來未見 } \\
\text { 過。 }\end{array}$ \\
\hline 51 & 重新 & cung $4 \operatorname{san} 1$ & $\mathrm{CM}$ & "again; anew" & 吓?又要重新做過呀? \\
\hline 52 & 從頭 & cung4tau4 & $\mathrm{CM}$ & "anew; once again" & 你從頭背一次叮！ \\
\hline 53 & 從中 & cung4zungl & $\mathrm{CM}$ & "from among" & 係唔係你响度從中搞鬼？ \\
\hline 54 & 打掂 & daa2dim 6 & $\mathrm{CS}$ & "vertically" & 張牀打橫放唔落, 打掂就得。 \\
\hline 55 & 打同 & daa2dung6 & $\mathrm{CS}$ & "vertically" & 啲木料應該打同放。 \\
\hline 56 & 打子子 & daa2maal & $\mathrm{CS}$ & "in pair" & 四支啤酒, 同我打孖綁叫。 \\
\hline 57 & 打橫 & daa2waang 4 & CS & "horizontally" & 張枱打橫放。 \\
\hline 58 & 大大 & daai6daai6 & $\mathrm{CM}$ & $\begin{array}{l}\text { "greatly; } \\
\text { enormously" }\end{array}$ & 無見兩年, 佢大大唔同咗。 \\
\hline 59 & 大大步 & daai6daai6bou6 & CS & "with wide steps" & 佢大大步追上去。 \\
\hline 60 & 大大啖 & daai6daai6daam6 & CS & "at a big mouthful" & 佢大大啖吞落肚。 \\
\hline 61 & 大大力 & daai6daai6lik6 & CS & "with a strong force" & 個細路仔大大力推開度門。 \\
\hline 62 & 大大聲 & daai6daai6sengl & CS & "very loudly" & 佢大大聲㖹我個名。 \\
\hline 63 & 大大話話 & daai6daai6waa6waa6 & CS & "approximately" & 哩度大大話話都有成千人。 \\
\hline 64 & 大淡大淡 & daai6daam6daai6daam6 & CS & "at a big mouthful" & $\begin{array}{l}\text { 個啤啤好鍾意牛奶, 大啖大 } \\
\text { 啖飲。 }\end{array}$ \\
\hline 65 & 大約 & daai6joek3 & $\mathrm{CM}$ & "approximately" & 哩班大約二十人。 \\
\hline 66 & 大嗱嗱 & daai6laa4laa4 & CS & "as much as" & $\begin{array}{l}\text { 大嗱嗱幾萬蚊, 我去邊度搵 } \\
\text { 啊？ }\end{array}$ \\
\hline 67 & 大力 & daai6lik6 & $\mathrm{CM}$ & "vigorously" & 間公司大力推銷呢隻牌紙。 \\
\hline 68 & 大略 & daai6loek2 & $\mathrm{CM}$ & "roughly" & 你大略講吓下你個構思喇。 \\
\hline 69 & 大體(上) & daai6tai2(soeng6) & $\mathrm{CM}$ & "on the whole" & 我大體上贊成你個講法。 \\
\hline
\end{tabular}




\begin{tabular}{|c|c|c|c|c|c|}
\hline 70 & 大致(上) & daai6zi3(soeng6) & $\mathrm{CM}$ & $\begin{array}{l}\text { "roughly; } \\
\text { approximately; more } \\
\text { or less" }\end{array}$ & 間學校大致上無咩改變。 \\
\hline 71 & 大早 & daai6zou2 & CS & "some time ago" & 你大早又唔講？ \\
\hline 72 & 單獨 & daanlduk6 & $\mathrm{CM}$ & "alone" & 佢單獨留响屋企度。 \\
\hline 73 & 單係 & daan1hai6 & CS & "only" & 我單係買衫都買咗成千蚊。 \\
\hline 74 & 第(二)日 & dai6(ji6)jat6 & CS & "later" & 我哋第(二)日再傾喇。 \\
\hline 75 & 第(二)時 & dai6(ji6)si4 & CS & "later; next time" & $\begin{array}{l}\text { 呢次比賽輸咗, 第(二)時再試 } \\
\text { 過。 }\end{array}$ \\
\hline 76 & 第一時間 & dai6jat1si4gaan 3 & CS & "immediately" & $\begin{array}{l}\text { 佢一入門口，就第一時間衝 } \\
\text { 咗入厠所。 }\end{array}$ \\
\hline 77 & 得滯 & daklzai6 & CS & "too" & $\begin{array}{l}\text { 個 present 悶得滯, 啲人咪走 } \\
\text { 晒囖。 }\end{array}$ \\
\hline 78 & 特別 & dak6bit6 & $\mathrm{CM}$ & "particularly" & 我今日特別好胃口。 \\
\hline 79 & 特登 & dak6dangl & $\mathrm{CS}$ & $\begin{array}{l}\text { "for a special } \\
\text { purpose; specially" }\end{array}$ & 你特登噉做咖！ \\
\hline 80 & 氹氹的緙 & dam4dam 4kwaak1 & $\mathrm{CS}$ & "in a circle" & 啲警察氹氹㗆圍住班劫匪。 \\
\hline 81 & 突然(之)間 & dat6jin4(zil)gaan 1 & $\mathrm{CS}$ & "suddenly" & $\begin{array}{l}\text { 我尋晚䀠莭吅下突然(之)間紮 } \\
\text { 醒咗。 }\end{array}$ \\
\hline 82 & 的(而且)確 & dikl(ji4ce2)kok3 & $\mathrm{CM}$ & "indeed; really" & 佢的確唔喺度住。 \\
\hline 83 & 點(不)知 & $\operatorname{dim} 2($ bat1)zil & CS & "unexpectedly" & 佢點(不)知唔黎得和。 \\
\hline 84 & 點止 & $\operatorname{dim} 2 z i 2$ & CS & "not only" & 去開會厩人點止十幾人啊。 \\
\hline 85 & 頂多 & ding $2 d o 1$ & $\mathrm{CM}$ & "at the most" & 佢頂多十五歲。 \\
\hline 86 & 頂籠 & ding2lung2 & CS & "at the most" & 呢度頂籠坐三十人。 \\
\hline 87 & 叠埋心水 & dip6maai4sam 1seoi2 & CS & $\begin{array}{l}\text { "wholeheartedly; } \\
\text { attentively" }\end{array}$ & 佢叠埋心水讀書。 \\
\hline 88 & 多多少少 & doldo1siu2siu2 & CS & "to a certain extent" & $\begin{array}{l}\text { 佢多多少少都知道自己唔 } \\
\text { 啱。 }\end{array}$ \\
\hline 89 & 多數 & dolsou3 & CS & $\begin{array}{l}\text { "probably; most } \\
\text { likely" }\end{array}$ & 雨咁大, 佢多數嚟唔到喇。 \\
\hline 90 & 當初 & donglcol & $\mathrm{CM}$ & "originally" & 佢當初點同你講㕵駕？ \\
\hline 91 & 當然 & dongljin4 & $\mathrm{CM}$ & "of course" & $\begin{array}{l}\text { 佢當然唔嚟喇，佢去咗美國 } \\
\text { 吅嗎。 }\end{array}$ \\
\hline 92 & 當面 & donglmin 2 & $\mathrm{CM}$ & $\begin{array}{l}\text { "in somebody's } \\
\text { presence }\end{array}$ & 大家當面講清楚至好。 \\
\hline 93 & 當堂 & dongltong 4 & $\mathrm{CS}$ & "immediately" & 佢當堂唔敢出聲。 \\
\hline 94 & 當临 & donglzung3 & $\mathrm{CM}$ & $\begin{array}{l}\text { "in the presence of } \\
\text { all" }\end{array}$ & 佢當篃閙佢個女。 \\
\hline 95 & 都 & doul & $\mathrm{CM}$ & $\begin{array}{l}\text { "after all; rather" } \\
\text { "also; as well" } \\
\text { "expressing } \\
\text { emphasis; already" } \\
\text { "all" } \\
\end{array}$ & \begin{tabular}{|l} 
件衫都唔錯。 \\
我都去。 \\
我都話唔得閒咯。 \\
人都走晒咯，做鬼做馬咩!
\end{tabular} \\
\hline 96 & 都係 & doulhai6 & $\mathrm{CS}$ & "had better" & 計我話都係留喺度好。 \\
\hline 97 & 到處 & dou $3 c y u 3$ & $\mathrm{CM}$ & "everywhere" & $\begin{array}{l}\text { 佢到處話自己幾叻幾叻，真 } \\
\text { 係無聊！ }\end{array}$ \\
\hline 98 & 到底 & dou3dai2 & $\mathrm{CM}$ & "actually; exactly" & 你到底係咩意思？ \\
\hline 99 & 到時 & dou3si4 & CS & "at that time" & 時間預鬆啲, 到時至唔會咁 \\
\hline
\end{tabular}




\begin{tabular}{|c|c|c|c|c|c|}
\hline & & & & & 趕。 \\
\hline 100 & 到頭黎 & dou3tau4lai4 & $\mathrm{CS}$ & "in the end; finally" & 佢到頭嚟輸哂啲錢。 \\
\hline 101 & 動不動 & dung6bat1dung6 & $\mathrm{CM}$ & "frequently" & 佢動不動就發脾氣。 \\
\hline 102 & 斷(斷) & dyun6(dyun6) & CS & "absolutely" & $\begin{array}{l}\text { 呢件事搵佢幫手，佢斷(斷) } \\
\text { 唔會托手蹊嘅。 }\end{array}$ \\
\hline 103 & 快啲 & faai3dil & CS & "quickly" & 仲唔快狛撤？ \\
\hline 104 & 快快脆脆 & faai3faai3ceoi3ceoi3 & CS & "quickly" & $\begin{array}{l}\text { 你一於快快脆脆食埋飯至 } \\
\text { 去。 }\end{array}$ \\
\hline 105 & 快手快腳 & faai3sau2faai3goek3 & CS & "quickly" & 佢快手快腳執埋啲野。 \\
\hline 106 & 反複 & faan $2 f u k l$ & $\mathrm{CM}$ & "repeatedly" & $\begin{array}{l}\text { 你反複諭吓將喵固樣嘢攞咗响 } \\
\text { 邊？ }\end{array}$ \\
\hline 107 & 反而 & faan $2 j i 4$ & $\mathrm{CM}$ & "on the contrary" & 佢叫我約人, 自己反而唔嚟。 \\
\hline 108 & 反爲 & faan 2 wal4 & $\mathrm{CM}$ & $\begin{array}{l}\text { "on the contrary; } \\
\text { instead" }\end{array}$ & 我反爲鍾意睇卡通片。 \\
\hline 109 & 反正 & faan2zing 3 & $\mathrm{CM}$ & "anyway" & 我幫你吅, 反正我又無堽做。 \\
\hline 110 & 凡親 & faan 4 can 1 & CS & "whenever" & 佢凡親出街, 都帶埋隻狗。 \\
\hline 111 & 分分鐘 & fanlfanlzung 1 & $\mathrm{CS}$ & "at any time" & 佢分分鐘會嚟搵你。 \\
\hline 112 & 分明 & fan1ming4 & $\mathrm{CM}$ & $\begin{array}{l}\text { "clearly; plainly; } \\
\text { evidently" }\end{array}$ & 佢日日遲到, 分明搏炒堵。 \\
\hline 113 & 分頭 & fanltau4 & $\mathrm{CM}$ & "separately" & 我哋分頭去嘍。 \\
\hline 114 & 分外 & fan6ngoi6 & $\mathrm{CM}$ & "particularly" & 佢今日分外客氣。 \\
\hline 115 & 忽然(之)間 & fatljin4(zil)gaanI & $\mathrm{CS}$ & "suddenly" & 隻狗忽然(之)間亂咁吠。 \\
\hline 116 & 非常(之) & fellsoeng4(zil) & $\mathrm{CM}$ & "very" & 我非常(之)多謝佢！ \\
\hline 117 & 逢(親) & fung4(can1) & CS & "whenever" & $\begin{array}{l}\text { 叔叔逢(親)食飯, 都要飲啤 } \\
\text { 酒。 }\end{array}$ \\
\hline 118 & 奉旨 & fung6zl2 & CS & "as a rule" & 佢好孤寒, 哩啲埋予奉旨唔買。 \\
\hline 119 & 加加埋埋 & gaalgaalmaai4maai4 & CS & "altogether" & $\begin{array}{l}\text { 我由屋企到哩度加加埋埋用 } \\
\text { 咗成個鐘。 }\end{array}$ \\
\hline 120 & 假假哋 & gaa2gaa2dei2 & CS & $\begin{array}{l}\text { "in any case; } \\
\text { anyhow" }\end{array}$ & $\begin{array}{l}\text { 佢假假吔都喺公司做咗咁 } \\
\text { 而。 }\end{array}$ \\
\hline 121 & 監粗 & gaam3coul & CS & "unyieldingly" & 監粗塞晒啲嘢入櫃桶度。 \\
\hline 122 & 監生 & gaam3saang 1 & CS & "while still alive" & 佢監生燒死咗。 \\
\hline 123 & 簡直 & gaan2zik6 & $\mathrm{CM}$ & "simply" & 哩間飯店簡直搶錢。 \\
\hline 124 & 間(唔)中 & gaan3(m4)zung 1 & CS & "occasionally" & 我間(唔)中搭吓巴士。 \\
\hline 125 & 夾硬 & gaap3ngaang2 & $\mathrm{CS}$ & "unyieldingly" & 夾硬迫人去。 \\
\hline 126 & 夾手夾腳 & gaap3sau2gaap 3 goek3 & CS & "jointly" & $\begin{array}{l}\text { 大家夾手夾腳將啲行李搬上 } \\
\text { 車。 }\end{array}$ \\
\hline 127 & 較爲 & gaau3wai4 & $\mathrm{CM}$ & "relatively" & 見到生保人，佢較爲靜。 \\
\hline 128 & 雞噉腳 & gailgam 2 goek3 & CS & "very quickly" & $\begin{array}{l}\text { 佢一見到經理嚟就雞噉腳走 } \\
\text { 嘞。 }\end{array}$ \\
\hline 129 & 計正 & gai3zeng3 & $\mathrm{CS}$ & $\begin{array}{l}\text { "under normal } \\
\text { circumstances; as a } \\
\text { rule" }\end{array}$ & 計正搭車應該快過行路。 \\
\hline 130 & 咁(鬼) & gam3(gwai2) & CS & "very" & $\begin{array}{l}\text { 你跑得咁(鬼)快, 我追唔到 } \\
\text { 你。 }\end{array}$ \\
\hline 131 & 咁啱 & gam3ngaam 1 & CS & "by chance" & 我琴日行公司, 咁啱大減價。 \\
\hline 132 & 咁滯 & gam3zai6 & CS & $\begin{array}{l}\text { "almost (positive } \\
\text { sentence)" }\end{array}$ & 啲錢用晒咁滯。 \\
\hline
\end{tabular}




\begin{tabular}{|c|c|c|c|c|c|}
\hline 133 & 根本(上) & gan1bun2(soeng6) & $\mathrm{CM}$ & "at all; simply" & 佢根本諗都末諗過。 \\
\hline 134 & 跟手 & gan1sau2 & $\mathrm{CM}$ & $\begin{array}{l}\text { "next; then; after } \\
\text { that" }\end{array}$ & $\begin{array}{l}\text { 一出咗西乿跟手就截的士 } \\
\text { 去, 啾得切嘅。 }\end{array}$ \\
\hline 135 & 跟住 & ganlzyu6 & CS & "afterwards; then" & 你行先, 我跟住嚟喫略。 \\
\hline 136 & 近乎 & $\operatorname{gan} 6 f u 4$ & $\mathrm{CM}$ & "close to" & 佢噉樣做近乎絕情。 \\
\hline 137 & 梗(係) & gang2(hai6) & CS & $\begin{array}{l}\text { "undoubtedly" } \\
\text { "certainly" }\end{array}$ & $\begin{array}{l}\text { 你跑响, 梗(係)快過我行咯! } \\
\text { 佢逢星期日梗(係)嚟哩度睇 } \\
\text { 書嘅。 }\end{array}$ \\
\hline 138 & 更加 & gang3gaal & $\mathrm{CM}$ & "even more" & 佢英文更加唔明喇。 \\
\hline 139 & 急急 & gaplgapl & $\mathrm{CS}$ & "nearly at once" & 佢急急打電話叫救傷車。 \\
\hline 140 & 急急腳 & gaplgaplgoek 3 & CS & "at a fast pace" & 佢急急腳走過噄。 \\
\hline 141 & 急急忙忙 & gaplgap1mong4mong4 & CS & $\begin{array}{l}\text { "in a hurry; in a } \\
\text { haste" }\end{array}$ & 佢急急忙忙走咗出去。 \\
\hline 142 & 急住 & gaplzyu6 & CS & $\begin{array}{l}\text { "in a hurry; in a } \\
\text { haste" }\end{array}$ & 你急住走咩? 坐而啲的忝喇。 \\
\hline 143 & 久唔久 & gau $2 m 4$ gau 2 & $\mathrm{CS}$ & "once in a while" & 我久唔久去睇戲。 \\
\hline 144 & 九成 & gau $2 \operatorname{sing} 4$ & $\mathrm{CS}$ & "very likely" & 我諗佢九成唔肯噄。 \\
\hline 145 & 夠 & $\operatorname{gan} 3$ & $\mathrm{CS}$ & "as well; too" & 佢怨我？我夠怨佢咯！ \\
\hline 146 & 究竟 & gau3ging2 & $\mathrm{CM}$ & "actually; exactly" & 你究竟想點？ \\
\hline 147 & 舊底 & gau6dai2 & CS & "in the past" & $\begin{array}{l}\text { 舊底你仲爭我五十蚊, 而家 } \\
\text { 仲想借? }\end{array}$ \\
\hline 148 & 基本上 & geilbun 2 soeng6 & $\mathrm{CM}$ & "basically" & 套戲基本上都唔錯。 \\
\hline 149 & 幾乎 & geilfu4 & $\mathrm{CM}$ & "almost" & 我幾乎俾駕車車親。 \\
\hline 150 & 幾 & gei2 & CS & \begin{tabular}{|l} 
"rather" \\
"very"
\end{tabular} & $\begin{array}{l}\text { 個後生仔幾醒喎。 } \\
\text { 佢今日心情幾靚啊！ }\end{array}$ \\
\hline 151 & 幾大 & gei2daai2 & CS & $\begin{array}{l}\text { "no matter (what; } \\
\text { how; etc.) }\end{array}$ & 佢幾大都要去。 \\
\hline 152 & 幾咁/幾鬼 & gei2gam3/gei2gwai2 & CS & "very" & 你睇佢幾咁開心啊！ \\
\hline 153 & 幾時得黎 & gei2si4dak1lai4 & CS & $\begin{array}{l}\text { "not within a period } \\
\text { of time" } \\
\text { "not (comparatively } \\
\text { speaking) }\end{array}$ & $\begin{array}{l}\text { 喵層樓幾時得嚟起好吅。 } \\
\text { 同母親節比，父親節幾時得 } \\
\text { 洯咁巴閉啊 。 }\end{array}$ \\
\hline 154 & 居然 & geoiljin4 & $\mathrm{CM}$ & "unexpectedly" & 佢居然食晒啲嘢喎！ \\
\hline 155 & 極 & gik6 & CS & $\begin{array}{l}\text { "extremely (before } \\
\text { adjective)" } \\
\text { "extremely (after } \\
\text { adjective)" } \\
\text { "repeatedly (after } \\
\text { action verb) } \\
\end{array}$ & $\begin{array}{l}\text { 極正；極好；極靚 } \\
\text { 佢啲字靚極都唔夠你寫得靚 } \\
\text { 喇。 } \\
\text { 食極都食唔晒。 }\end{array}$ \\
\hline 156 & 極其量 & gik6kei4loeng6 & CS & "at the most" & 啲代表極其量得二百人。 \\
\hline 157 & 極力 & gik6lik6 & $\mathrm{CM}$ & "do one's utmost" & 佢極力勸我唔好買哩樣理予。 \\
\hline 158 & 極之 & gik6zil & CS & "extremely" & 哩齣戲極之好睇。 \\
\hline 159 & 經已 & ginglji5 & CS & "already" & 佢經已有咗三個月身紀。 \\
\hline 160 & 竟然 & ging2jin4 & $\mathrm{CM}$ & "unexpectedly" & 你竟然唔出街, 少有喎！ \\
\hline 161 & 勁 & ging6 & CS & "extremely" & 啲魚蛋勁辣啊！ \\
\hline 162 & 各自各 & gok3zi6gok3 & CS & "respectively" & 我此各自各返屋企。 \\
\hline 163 & 乾 & gon1 & $\mathrm{CM}$ & "in a dry manner" & 乾洗；乾炒 \\
\hline 164 & 乾脆 & gon1ceoi3 & CS & "simply" & 佢爲求套現, 乾脆賣咗間屋。 \\
\hline
\end{tabular}




\begin{tabular}{|c|c|c|c|c|c|}
\hline 165 & 姑且 & gulce2 & $\mathrm{CM}$ & "for the time being" & 我姑且信你一次。 \\
\hline 166 & 固然之 & gu3jin $4 z i l$ & CS & "no doubt" & $\begin{array}{l}\text { 噉做固然之好, 只不過係怕 } \\
\text { 佢唔鍾意啫。 }\end{array}$ \\
\hline 167 & 公然 & gungljin4 & $\mathrm{CM}$ & "openly" & 但公然破壞咗大家眼協議。 \\
\hline 168 & 鬼 & gwai2 & CS & "not" & 我理鬼佢。 \\
\hline 169 & 鬼咁 & gwai2gam 3 & $\mathrm{CS}$ & "very" & 佢鬼咁䖯。 \\
\hline 170 & 滾水淥腳(噉) & gwan2seoi2luk6goek3(gam2) & CS & $\begin{array}{l}\text { "in a hurry as if one } \\
\text { were burnt by hot } \\
\text { water on the leg" }\end{array}$ & 佢滾水淥腳(噉)走咗。 \\
\hline 171 & 果然 & gwo2jin4 & $\mathrm{CM}$ & "accordingly" & 佢話唔走住, 果然無走到。 \\
\hline 172 & 過分 & gwo3fan6 & $\mathrm{CM}$ & "excessively" & 咪過分緊張喇! \\
\hline 173 & 過後 & gwo3hau6 & $\mathrm{CM}$ & "afterwards; later" & $\begin{array}{l}\text { 佢而家話會做, 過後又唔記 } \\
\text { 得嘫鐴。 }\end{array}$ \\
\hline 174 & 過頭 & gwo3tau4 & CS & "very; too" & 哩度熱過頭。 \\
\hline 175 & 閒閒哋 & haan4haan2dei2 & CS & "very easily" & $\begin{array}{l}\text { 佢咁有錢, 閒閒哋攞幾百萬 } \\
\text { 出洯喇。 }\end{array}$ \\
\hline 176 & 住度敃處 & hai2dou6/hai2syu3 & CS & "in progress of" & 伯嗦度係噉食係噉食。 \\
\hline 177 & 係都 & hai6doul & CS & "with insistence" & 佢係都跟埋嚟。 \\
\hline 178 & 係噉 & hai6gam2 & CS & "continuously" & 佢淨係係噉笑。 \\
\hline 179 & 係噉意 & hai6gam $2 j i 2$ & CS & "perfunctorily" & 佢係噉意回番封信。 \\
\hline 180 & 刻意 & haklji3 & $\mathrm{CM}$ & "painstakingly" & $\begin{array}{l}\text { 嗰個明星刻意表現到好自 } \\
\text { 然。 }\end{array}$ \\
\hline 181 & Ш蹦呤 & ham6baang6laang6 & CS & "all" & $\begin{array}{l}\text { 我將狛的野宁蹋呤拎晒番屋 } \\
\text { 企。 }\end{array}$ \\
\hline 182 & 肯定 & hang2ding6 & $\mathrm{CM}$ & "surely" & 佢聽日肯定嚟唔到。 \\
\hline 183 & 口快快(噉) & hau2faai3faai3(gam2) & CS & "without hesitation" & 點知任口快快(噉)講咗出嚟。 \\
\hline 184 & 口ロ聲聲 & hau 2 hau 2 seng 1 sengl & $\mathrm{CM}$ & $\begin{array}{l}\text { "(say) again and } \\
\text { again" }\end{array}$ & 佢口口聲聲話佢無做過。 \\
\hline 185 & 後尾 & hau6meil & CS & "afterwards; later" & 佢後尾系离咗線。 \\
\hline 186 & 起初 & hei2col & $\mathrm{CM}$ & "originally" & $\begin{array}{l}\text { 哩間廠起初好細，點知越做 } \\
\text { 越大。 }\end{array}$ \\
\hline 187 & 起碼 & hei2maa5 & $\mathrm{CM}$ & "at least" & 佢噉樣講我咃起碼安落啲。 \\
\hline 188 & 起勢 & hei2sai3 & CS & "desperately" & 佢起勢掟枝筆落地下。 \\
\hline 189 & 起先 & hei $2 \sin 1$ & CS & "at the beginning" & $\begin{array}{l}\text { 佢起先話去, 不過琴日病 } \\
\text { 咗, 唔去得辚。 }\end{array}$ \\
\hline 190 & 輕輕 & heng6heng1 & CS & "slightly" & 隻袖輕輕長番狛的就好倁虚。 \\
\hline 191 & 輕易 & hinglji6 & $\mathrm{CM}$ & "rashly" & 佢唔輕易讚人騭！ \\
\hline 192 & 可能 & ho2nang4 & $\mathrm{CM}$ & "probably" & 聽日可能會落雨。 \\
\hline 193 & 何必 & ho4bit1 & $\mathrm{CM}$ & "why bother" & 輸贏何必咁認真？ \\
\hline 194 & 何苦 & ho4fu2 & $\mathrm{CM}$ & "why bother" & 你何苦同佢拗呢？ \\
\hline 195 & 何止 & ho4zi2 & $\mathrm{CS}$ & "not only" & $\begin{array}{l}\text { 講真叮, 任技術上何止勝你 } \\
\text { 一书, 幾皮啊。 }\end{array}$ \\
\hline 196 & 响度晌處 & hoeng2dou6/hoeng2syu3 & $\mathrm{CS}$ & "in progress of" & $\begin{array}{l}\text { 你睇個天响度猛咁閃電, 就 } \\
\text { 嫩落大雨唓。 }\end{array}$ \\
\hline 197 & 向來 & hoeng3loi4 & $\mathrm{CM}$ & "always" & 我向來唔飲酒。 \\
\hline 198 & 開口埋口 & hoilhau2maai4hau2 & CS & $\begin{array}{l}\text { "always talking about } \\
\text { the same thing" }\end{array}$ & 佢開口埋口淨係識講錢。 \\
\hline 199 & 好(鬼) & hou2(gwai2) & CS & "extremely" & 我好(鬼)肚餓。 \\
\hline
\end{tabular}




\begin{tabular}{|c|c|c|c|c|c|}
\hline 200 & 好似 & hou $2 c i 5$ & $\mathrm{CM}$ & "presumably" & 哩枝筆好似唔係我喵枝。 \\
\hline 201 & 好多時 & hou2do1si4 & CS & "very often" & 佢好多時唔記得帶鎖匙。 \\
\hline 202 & 好好(吅) & hou2hou2(dei2) & $\mathrm{CS}$ & "well" & 你要好好(呲)對人啊。 \\
\hline 203 & 好好醜醜 & hou 2 hou 2 cau 2 cau2 & CS & $\begin{array}{l}\text { "in any case; } \\
\text { whatever happens" }\end{array}$ & $\begin{array}{l}\text { 你好好醜醜都做完酔至走 } \\
\text { 叮。 }\end{array}$ \\
\hline 204 & 好日 & hou2jat6 & CS & $\begin{array}{l}\text { "rarely; seldom } \\
\text { (negative sentence)" }\end{array}$ & 我好日都唔運動。 \\
\hline 205 & 好意思 & hou2ji3sil & $\mathrm{CS}$ & "have the nerve" & 你好意思唔應承佢咩？ \\
\hline 206 & 好唔好都 & hou $2 m 4$ hou 2 doul & CS & $\begin{array}{l}\text { "in any case; } \\
\text { whatever happens" }\end{array}$ & $\begin{array}{l}\text { 你好唔好都做住呢份工先 } \\
\text { 喇。 }\end{array}$ \\
\hline 207 & 好少 & hou $2 \sin 2$ & CS & "rarely; seldom" & 佢近排好少嚟探我哋。 \\
\hline 208 & 好在 & hou2zoi6 & $\mathrm{CM}$ & "fortunately" & 佢好在趕得切嫩瑡。 \\
\hline 209 & - & jat1 & $\mathrm{CM}$ & "as soon as" & 佢一返嚟就鬫人。 \\
\hline 210 & 一邊... 一邊.. & jatlbin1...jatlbinl ... & $\mathrm{CM}$ & "at the same time" & 佢一邊食飯一邊講嘢。 \\
\hline 211 & 一便...一便.. & jat1bin6...jat1bin6... & CS & "at the same time" & 佢一便睇一便笑。 \\
\hline 212 & 一步步 & jat1bou2bouб & CS & $\begin{array}{l}\text { "step by step; } \\
\text { progressively" }\end{array}$ & $\begin{array}{l}\text { 我吔一步步改善緊教育質素 } \\
\text { 呰嘞。 }\end{array}$ \\
\hline 213 & 一齊 & jatlcai4 & $\mathrm{CM}$ & $\begin{array}{l}\text { "together" } \\
\text { "along with all the } \\
\text { others" } \\
\text { "at a time" }\end{array}$ & 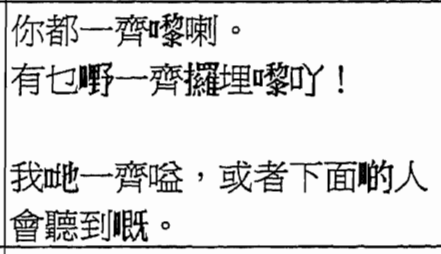 \\
\hline 214 & 一次又一次 & jat1ci3jau6jatlci3 & CS & $\begin{array}{l}\text { "time and again; } \\
\text { repeatedly" }\end{array}$ & 佢一次又一次呃我。 \\
\hline 215 & 一旦 & jat1daan6 & $\mathrm{CM}$ & "once; in case" & 哩件事一旦爆保, 就不得了。 \\
\hline 216 & 一定 & jat1ding 6 & $\mathrm{CM}$ & "certainly; surely" & $\begin{array}{l}\text { 每一個人都一定有好朋友 } \\
\text { 鴜。 }\end{array}$ \\
\hline 217 & 一度 & jat1dou6 & $\mathrm{CM}$ & "for a time" & 佢一度停學咗成年。 \\
\hline 218 & 一共 & jatlgung6 & $\mathrm{CM}$ & "altogether" & 哩度一共四千蚊。 \\
\hline 219 & 一口 & jat1hau2 & $\mathrm{CM}$ & "with certainty" & 佢一口咬定係我做溉。 \\
\hline 220 & 一口價 & jatl hau2gaa 3 & CS & "at a fixed price" & $\begin{array}{l}\text { 你有心買蔇話，我一口價十 } \\
\text { 蚊賣俾你。 }\end{array}$ \\
\hline 221 & 一口氧 & jatlhau2hei3 & $\mathrm{CS}$ & $\begin{array}{l}\text { "in one breath; } \\
\text { without a break" }\end{array}$ & $\begin{array}{l}\text { 佢馨起嚟一口氣唱咗十幾隻 } \\
\text { 歌。 }\end{array}$ \\
\hline 222 & 一向 & jatlhoeng3 & $\mathrm{CM}$ & $\begin{array}{l}\text { "consistently; all } \\
\text { along" }\end{array}$ & 你一向鍾意食辣。 \\
\hline 223 & 二- & jat1jat1 & $\mathrm{CM}$ & "one by one" & 我哋一一自我介紹。 \\
\hline 224 & 一日 & jatljat6 & CS & $\begin{array}{l}\text { "in the final analysis; } \\
\text { at bottom" }\end{array}$ & 哩件事一日都係你唔啱。 \\
\hline 225 & 一日到黑 & jat1jat6dou3hakl & CS & $\begin{array}{l}\text { "from morning till } \\
\text { night; all day long" } \\
\text { "always" }\end{array}$ & $\begin{array}{l}\text { 一日到黑睇住間鋪。 } \\
\text { 佢一日到黑話人呃佢。 }\end{array}$ \\
\hline 226 & 一於 & jat1jyul & $\mathrm{CS}$ & $\begin{array}{l}\text { "regardless of the } \\
\text { consequences" } \\
\text { "simply; just; } \\
\text { altogether" } \\
\text { "expressing decision" }\end{array}$ & 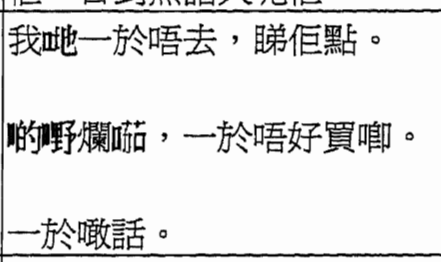 \\
\hline 227 & 一概 & jatlkoi3 & $\mathrm{CM}$ & "without exception; & 哩啲嘢佢一概唔答。 \\
\hline
\end{tabular}




\begin{tabular}{|c|c|c|c|c|c|}
\hline & & & & totally" & \\
\hline 228 & 一輪嘴 & jat1leon2zeoi2 & $\mathrm{CS}$ & "without a break" & $\begin{array}{l}\text { 佢將啲事實一輪嘴講晒出 } \\
\text { 嚟。 }\end{array}$ \\
\hline 229 & 一律 & jatlleot6 & $\mathrm{CM}$ & "without exception" & $\begin{array}{l}\text { 大人細路一律二十蚊入場 } \\
\text { 費。 }\end{array}$ \\
\hline 230 & 一連 & jat1lin4 & $\mathrm{CM}$ & "in succession" & 一連三日落大雨。 \\
\hline 231 & 一路 & jat1lou6 & $\mathrm{CM}$ & $\begin{array}{l}\text { "continuously; } \\
\text { always; all along; all } \\
\text { the way" } \\
\text { "at the same time; } \\
\text { simultaneously" }\end{array}$ & $\begin{array}{l}\text { 我吔一路都係噉做嘢嗎喇。 } \\
\text { 我吔一路飲一路傾。 }\end{array}$ \\
\hline 232 & 一味 & jat1mei2 & $\mathrm{CM}$ & "blindly; endlessly" & 你一味掛住玩。 \\
\hline 233 & 一面...一面 $\ldots$ & jatlmin $6 . . . j a t 1 m i n 6$. & $\mathrm{CM}$ & "at the same time" & 佢一面笑一面講。 \\
\hline 234 & 一眼 & jatlngaan 5 & $\mathrm{CM}$ & "in a glimpse" & 佢一眼認出我。 \\
\hline 235 & 一心 & jatlsam 1 & $\mathrm{CM}$ & $\begin{array}{l}\text { "wholeheartedly" } \\
\text { "intentionally" }\end{array}$ & $\begin{array}{l}\text { 我一心彗佢, 點知佢唔領情。 } \\
\text { 佢一心同我作對。 }\end{array}$ \\
\hline 236 & 一手 & jat1sau2 & $\mathrm{CM}$ & "single-handed" & 佢一手就拎起張橙。 \\
\hline 237 & 一手一腳 & jat1sau2jatlgoek3 & CS & "by oneself" & 佢自己一手一腳做蔇。 \\
\hline 238 & 一時時 & jat1si2si4 & CS & "occasionally" & 佢一時時擎探我嗎。 \\
\hline 239 & 一時(之間) & jat1si4(zilgaanl) & CS & "for the time being" & $\begin{array}{l}\text { 我一時(之間)諗唔起佢係邊 } \\
\text { 個。 }\end{array}$ \\
\hline 240 & 一時...一時... & jat1si4...jatIsi4... & CS & $\begin{array}{l}\text { "sometimes ...; } \\
\text { sometimes..." }\end{array}$ & \begin{tabular}{|l} 
呢排蔇天氣真係無譜, 一時 \\
熱到死, 一時又凍到死。
\end{tabular} \\
\hline 241 & 一䁪眼 & jat1zaam2ngaan 5 & CS & "in an instant" & 佢一䁪眼就唔見咗人。 \\
\hline 242 & 一直 & jatlzik6 & $\mathrm{CM}$ & "continuously" & $\begin{array}{l}\text { 佢間公司幾年嚟一直好順 } \\
\text { 利。 }\end{array}$ \\
\hline 243 & 一再 & jatlzoi3 & $\mathrm{CM}$ & "time and again" & 佢一再強調佢無做過。 \\
\hline 244 & 一早 & jatlzou2 & CS & "in advance" & 我吔一一早買咗飛喇。 \\
\hline 245 & 日日 & jat6jat6 & $\mathrm{CM}$ & "daily; regularly" & 佢日日遲到，分明搏炒啫。 \\
\hline 246 & 日夜 & jat6je6 & $\mathrm{CM}$ & "day and night" & 啲警察日夜眀住佢。 \\
\hline 247 & 由頭 & jau4tau4 & CS & "from the beginning" & 我哋一齊由頭讀一次。 \\
\hline 248 & 有 & jau5 & CS & "surely; already" & 佢有打電話嚟。 \\
\hline 249 & 有得 & jau5dakl & $\mathrm{CS}$ & $\begin{array}{l}\text { "it is possible to" } \\
\text { "it is worthwhile to" }\end{array}$ & $\begin{array}{l}\text { 我哋有得食海鮮。 } \\
\text { 哩個問題有得諗。 }\end{array}$ \\
\hline 250 & 有啲 & jau5dil & CS & "a little bit" & 我有啲唔舒服。 \\
\hline 251 & 有幾何 & jau5gei2ho2 & CS & \begin{tabular}{|l|} 
"rarely; seldom \\
(rhetorical sentence)"
\end{tabular} & 你有幾何嚟到呋, 坐多陣喇。 \\
\hline 252 & 有意 & jau5ji3 & CS & $\begin{array}{l}\text { "intentionally; } \\
\text { deliberately" }\end{array}$ & 你有意同我作對！ \\
\hline 253 & 有理無理 & jau5lei5mou5lei5 & CS & $\begin{array}{l}\text { "regardless of the } \\
\text { consequences" }\end{array}$ & 我有理無理做咗先算。 \\
\hline 254 & 有時 & jau5si4 & $\mathrm{CM}$ & "sometimes" & 我個人有時好失魂鴜。 \\
\hline 255 & 有陣時 & jau5zan6si2 & CS & "sometimes" & 我有陣時一個人落街行吓。 \\
\hline 256 & 又 & jau6 & $\mathrm{CM}$ & $\begin{array}{l}\text { "repeatedly; again" } \\
\text { "at the same time" } \\
\text { "also; expressing } \\
\text { information of the } \\
\text { same kind" }\end{array}$ & \begin{tabular}{|l|} 
睇完又睇。 \\
佢做野又快又好。 \\
食完飯, 又無䧉做，不如出 \\
去行吓。
\end{tabular} \\
\hline
\end{tabular}




\begin{tabular}{|c|c|c|c|c|c|}
\hline & & & & $\begin{array}{l}\text { "but" } \\
\text { "expressing } \\
\text { emphasis" }\end{array}$ & $\begin{array}{l}\text { 我想食糖，又怕肥。 } \\
\text { 我又無做過，驚咩唧。 }\end{array}$ \\
\hline 257 & 又試 & jau6si3 & CS & "again" & 佢又試食零食。 \\
\hline 258 & 依稀 & jilheil & $\mathrm{CM}$ & "vaguely" & 我依稀記得佢嚟過。 \\
\hline 259 & 依然 & jiljin4 & $\mathrm{CM}$ & "still" & 佢依然日日嫩。 \\
\hline 260 & 依時(依候) & jilsi4(jilhau6) & CS & "punctually" & 我依時(依候)食葯。 \\
\hline 261 & 已經 & ji5ging1 & $\mathrm{CM}$ & "already" & 佢已經走咗蹽。 \\
\hline 262 & 異常 & ji6soeng 4 & $\mathrm{CM}$ & "extremely" & 佢今日異常興舊。 \\
\hline 263 & 亦(都) & jik6(doul) & $\mathrm{CM}$ & "as well" & $\begin{array}{l}\text { 佢主要讀經濟，會計亦(都) } \\
\text { 識啲咖茄。 }\end{array}$ \\
\hline 264 & 現皃皃 & jin6dauldaul & CS & "in cash" & $\begin{array}{l}\text { 我現兒淣用咗三百蚊買番嚟 } \\
\text { 蔇。 }\end{array}$ \\
\hline 265 & 應份 & jingl fan 6 & CS & "necessarily" & 呢筆錢我應份俾萔。 \\
\hline 266 & 認真 & jing $2 z a n 1$ & CS & "absolutely" & 佢做嘢認真快。 \\
\hline 267 & 仍然 & jng4jin4 & $\mathrm{CM}$ & "still; yet" & 我仍然未搵到工。 \\
\hline 268 & 約莫 & joek 3 mok2 & $\mathrm{CM}$ & "approximately" & 佢約莫三十幾歲。 \\
\hline 269 & 郁啲 & jukldil & CS & "easily; frequently" & 佢有队啲就喊。 \\
\hline 270 & 郁吓下 & juklhaa5 & $\mathrm{CS}$ & "easily; frequently" & 佢有吅下就鬧人。 \\
\hline 271 & 預先 & $j y u 6 \sin 1$ & $\mathrm{CM}$ & $\begin{array}{l}\text { "in advance; } \\
\text { beforehand" }\end{array}$ & $\begin{array}{l}\text { 我預先過咗啲料偅佢，等佢 } \\
\text { 有心理準備。 }\end{array}$ \\
\hline 272 & 預早 & jyu6zou2 & CS & $\begin{array}{l}\text { "in advance; } \\
\text { beforehand" }\end{array}$ & 你幾時走, 要預早話低。 \\
\hline 273 & 完全 & jyun4cyun4 & $\mathrm{CM}$ & "completely" & 佢份功課完全做錯晒。 \\
\hline 274 & 原來 & jyun4loi4 & $\mathrm{CM}$ & "originally" & 佢原來病咗, 無返工。 \\
\hline 275 & 原先 & jyun $4 \sin 1$ & $\mathrm{CM}$ & "originally" & 佢原先話去, 而家又唔去。 \\
\hline 276 & 遠遠 & jyun 5jyun5 & $\mathrm{CM}$ & "largely" & 你遠遠比唔上佢勤力。 \\
\hline 277 & 越...越... & jyut6...jyut6... & $\mathrm{CM}$ & $\begin{array}{l}\text { "the more...the } \\
\text { more..." }\end{array}$ & 我越諗就越㷫。 \\
\hline 278 & 越敕越 & jyut6lai4jyut6 & CS & "more and more" & 越黎越多人知道哩件事。 \\
\hline 279 & 卡那(卡那) & kaa3laal (kaa3laal) & CS & "alternatively" & 我哋卡那(卡那)噉坐。 \\
\hline 280 & 捴捴青 & kam4kam2ceng1 & CS & "quickly" & 我擒擒青走番入屋。 \\
\hline 281 & 勤的 & kan4dil & $\mathrm{CS}$ & "frequently" & 夏天啲衫要勤啲洗。 \\
\hline 282 & 及早 & kap6zou2 & $\mathrm{CM}$ & "as soon as possible" & 你都係及早去睇醫生喇。 \\
\hline 283 & 其實 & kei4sat6 & $\mathrm{CM}$ & "actually; in fact" & 揸車其實都唔係好難啫。 \\
\hline 284 & 確係 & kok3hai6 & $\mathrm{CS}$ & "really; indeed" & 佢確係無嚟過。 \\
\hline 285 & 確實 & kok3sat6 & $\mathrm{CM}$ & "really; indeed" & 我確實唔知道。 \\
\hline 286 & 狂 & kwong4 & CS & "crazily" & \begin{tabular}{|l} 
佢狂飲啤酒卒之搞到入醫 \\
院。
\end{tabular} \\
\hline 287 & 搼埋 & laa2maai4 & CS & $\begin{array}{l}\text { "easily; frequently; at } \\
\text { every turn" } \\
\text { "generally speaking" }\end{array}$ & $\begin{array}{l}\text { 你唔好嗱埋就發舅，㯖佢解 } \\
\text { 釋先。 } \\
\text { 哩隊籃球隊嘅球員個個撑埋 } \\
\text { 都有兩米以上。 }\end{array}$ \\
\hline 288 & 嗱(嗱)臨 & laa4(laa4)lam4 & CS & $\begin{array}{l}\text { "very quickly; at } \\
\text { once" }\end{array}$ & 你嗱(嗱)臨返學先喇。 \\
\hline 289 & 嗱嗱聲 & laa4laa2seng1 & $\mathrm{CS}$ & $\begin{array}{l}\text { "very quickly; at } \\
\text { once" }\end{array}$ & $\begin{array}{l}\text { 我地嗱嗱聲做埋啲嘢就走 } \\
\text { 嘞。 }\end{array}$ \\
\hline 290 & 拉匀 & laailwan4 & CS & "on average" & 間鋪頭每年拉匀都賺到十幾 \\
\hline
\end{tabular}




\begin{tabular}{|c|c|c|c|c|c|}
\hline & & & & & 萬鴜。 \\
\hline 291 & 䊖日 & laam3jat6 & $\mathrm{CS}$ & "every two days" & $\begin{array}{l}\text { 佢唔係日日敕, 䊖日先嫩一 } \\
\text { 次。 }\end{array}$ \\
\hline 292 & 立即 & laap6zik1 & $\mathrm{CM}$ & "immediately" & 一㯖完電話, 佢立即走咗。 \\
\hline 293 & 臨急臨忙 & lam4gap 1lam4mong4 & CS & "at the last moment" & $\begin{array}{l}\text { 我臨急臨忙搵咗佢嚟頂替 } \\
\text { 我。 }\end{array}$ \\
\hline 294 & 臨時臨急 & lam4si4lam4gapI & CS & "at the last moment" & 佢臨時臨急先話唔黎。 \\
\hline 295 & 笠亂 & lap6lyun2 & CS & $\begin{array}{l}\text { "carelessly; casually; } \\
\text { at random" }\end{array}$ & 哩啲說話唔好笠亂講。 \\
\hline 296 & 輪流 & leon4lau2 & CS & "in turn" & 我呲輪流照顧阿爸。 \\
\hline 297 & 哩哩啦啦 & li4li1laa4laa4 & CS & "very quickly" & $\begin{array}{l}\text { 我哋咁多人, 哩哩啦啦就做 } \\
\text { 完晒咯。 }\end{array}$ \\
\hline 298 & 力 & lik6 & $\mathrm{CM}$ & "make every effort" & 力捧 ; 力勸 \\
\hline 299 & 鏈住條頸 & lin2zyu6tiu4geng2 & $\mathrm{CS}$ & "unwillingly" & 咪鏈住條頸聽佢唱 $\mathrm{K}$ 筫。 \\
\hline 300 & 連隨 & $\operatorname{lin} 4$ ceoi4 & CS & "immediately" & $\begin{array}{l}\text { 喵晚黑一落火車, 連隨就搭 } \\
\text { 船返番江門。 }\end{array}$ \\
\hline 301 & 連氣 & lin4hei3 & $\mathrm{CS}$ & "in succession" & 佢連氣食咗三大碗麵。 \\
\hline 302 & 連續 & $\operatorname{lin} 4 z u k 6$ & $\mathrm{CM}$ & "successively" & 我連續問咗佢三個問題。 \\
\hline 303 & 靈舍 & ling 4 se 3 & CS & "particularly" & 今日去睇波嘅人靈舍多。 \\
\hline 304 & 另外 & ling6ngoi6 & $\mathrm{CM}$ & "in addition" & 我另外叫咗碟沙律。 \\
\hline 305 & 略略 & loek6loek2 & $\mathrm{CM}$ & $\begin{array}{l}\text { "a little; a bit; } \\
\text { slightly" }\end{array}$ & 張枱略略移開啲咪得囖。 \\
\hline 306 & 略爲 & loek6wai4 & $\mathrm{CM}$ & "slightly" & 件衫略爲大咗睹！ \\
\hline 307 & 兩份 & loeng5fan2 & CS & "together" & 呢包餅你同細佬兩份食。 \\
\hline 308 & 來回 & loi4wui4 & $\mathrm{CM}$ & "back and forth" & 佢來回嚟咗醫院三次。 \\
\hline 309 & 落手 & lok6sau2 & CS & $\begin{array}{l}\text { "by oneself; in } \\
\text { person" }\end{array}$ & 細妹今日落手整蛋糕。 \\
\hline 310 & 落手落腳 & lok6sau2lok6goek3 & CS & $\begin{array}{l}\text { "by oneself; in } \\
\text { person" }\end{array}$ & $\begin{array}{l}\text { 整餤最最好自己落手落腳去 } \\
\text { 整。 }\end{array}$ \\
\hline 311 & 陸陸續續 & luk6luk6zuk6zuk6 & $\mathrm{CM}$ & "in succession" & 啲人陸陸續續嚟到劇場。 \\
\hline 312 & 陸續 & luk6zuk6 & $\mathrm{CM}$ & "in succession" & 我哋陸續返番屋企。 \\
\hline 313 & 亂(咁) & lyun2(gam3) & $\mathrm{CM}$ & "thoughtlessly" & 你咪亂(咁)倹浻！ \\
\hline 314 & 唔(鬼) & $m 4$ (gwai2) & $\mathrm{CS}$ & "not" & 你唔去呀? \\
\hline 315 & 唔...又. & $m 4 \ldots$ jau $6 \ldots$ & $\mathrm{CS}$ & "not...but" & 你唔食又食。 \\
\hline 316 & 唔多 & $m 4 d o l$ & CS & "not very" & 佢哋丽個唔多䘽。 \\
\hline 317 & 唔多唔少 & $m 4 d o 1 m 4 \operatorname{siu} 2$ & $\mathrm{CS}$ & "to a certain extent" & $\begin{array}{l}\text { 噉做法唔多唔少有啲作用 } \\
\text { 既。 }\end{array}$ \\
\hline 318 & 唔慌 & $m 4$ fong 1 & CS & "absolutely not" & 佢唔慌係好人。 \\
\hline 319 & 唔見得 & m4gin 3 dakl & $\mathrm{CS}$ & "not likely" & 佢唔見得鍾意你喎。 \\
\hline 320 & 唔經唔覺 & m4ging Im4gok3 & CS & $\begin{array}{l}\text { "without knowing } \\
\text { consciously" }\end{array}$ & 唔經唔覺幾十歲咯！ \\
\hline 321 & 唔怪(之)得 & m4gwaai3(zil)dakl & CS & "that is why" & $\begin{array}{l}\text { 唔怪(之)得佢唔去喇, 原來佢 } \\
\text { 有事。 }\end{array}$ \\
\hline 322 & 唔好 & m4hou2 & $\mathrm{CS}$ & "don't" & 你唔好喊喇。 \\
\hline 323 & 唔使 & m4sai2 & CS & "not necessarily" & 你唔使咁快決定葀。 \\
\hline 324 & 唔使問 & $m 4 \operatorname{sai} 2 \operatorname{man} 6$ & $\mathrm{CS}$ & "undoubtedly" & 佢唔使問又遲到喇！ \\
\hline 325 & 唔使審 & m4sai2sam2 & CS & "undoubtedly" & $\begin{array}{l}\text { 你唔使審去咗玩, 無做到功 } \\
\text { 課。 }\end{array}$ \\
\hline
\end{tabular}




\begin{tabular}{|c|c|c|c|c|c|}
\hline 326 & 唔聲唔聲 & $m 4$ seng $1 m 4$ seng 1 & CS & "quietly" & $\begin{array}{l}\text { 佢唔聲唔聲行埋嚟，哧咗我 } \\
\text { 一跳。 }\end{array}$ \\
\hline 327 & 唔通 & m4tungl & CS & $\begin{array}{l}\text { "expressing } \\
\text { rhetorical question" } \\
\text { "can it be that; is it } \\
\text { possible that" }\end{array}$ & $\begin{array}{l}\text { 唔通你惡, 我就要怕咗你? } \\
\text { 把遮唔通俾人撻咗? }\end{array}$ \\
\hline 328 & 唔掙在 & m4zaanglzoi6 & $\mathrm{CS}$ & "never mind" & $\begin{array}{l}\text { 架車咁大, 唔掙在坐多幾個 } \\
\text { 人喇。 }\end{array}$ \\
\hline 329 & 唔知 & $m 4 z i 1$ & $\mathrm{CS}$ & $\begin{array}{l}\text { "unknowingly" } \\
\text { "really" }\end{array}$ & $\begin{array}{l}\text { 佢唔知行咗去邊。 } \\
\text { 落雨唔打得波, 唔知幾無 } \\
\text { 瘝！ }\end{array}$ \\
\hline 330 & 唔只 & $m 4 z i 2$ & $\mathrm{CS}$ & "not only" & 佢唔只呛我, 仲呃埋你。 \\
\hline 331 & $\begin{array}{l}\text { 唔淨只/ } \\
\text { 唔單只 }\end{array}$ & $\begin{array}{l}\text { m4zing6zi2/ } \\
\text { m4daan16zi2 }\end{array}$ & $\mathrm{CS}$ & "not only" & $\begin{array}{l}\text { 我哋唔淨只去行街, 仲去睇 } \\
\text { 戲啔。 }\end{array}$ \\
\hline 332 & 㮸 & $m 6$ & $\mathrm{CM}$ & "mistakenly" & 誤殺; 誤食 \\
\hline 333 & 漫 & maan6 & $\mathrm{CM}$ & "casually" & 漫談；漫步 \\
\hline 334 & 萬二分 & maan6jibfanl & $\mathrm{CS}$ & $\begin{array}{l}\text { "very much; } \\
\text { extremely" }\end{array}$ & 我萬二分感激你。 \\
\hline 335 & 慢慢 & maan6maan2 & $\mathrm{CS}$ & "slowly" & 我慢慢行。 \\
\hline 336 & 猛(咁) & maang5(gam3) & $\mathrm{CS}$ & "desperately" & 我猛(咁)跑都趕唔切。 \\
\hline 337 & 咪 & mai5 & $\mathrm{CS}$ & "don't" & 你咪有啊, 等埋我。 \\
\hline 338 & 咪 & mai6 & $\mathrm{CS}$ & $\begin{array}{l}\text { "exactly; really" } \\
\text { "then" }\end{array}$ & $\begin{array}{l}\text { 佢咪輸咗囖。 } \\
\text { 你鍾意咪攞去囖。 }\end{array}$ \\
\hline 339 & 也滯 & mat1zai6 & $\mathrm{CS}$ & $\begin{array}{l}\text { "not quite (negative } \\
\text { sentence)" }\end{array}$ & 我都無用七滯。 \\
\hline 340 & 貿然 & $\operatorname{mau6jin} 4$ & $\mathrm{CM}$ & "rashly; hastily" & 你唔可以貿然下結論。 \\
\hline 341 & 貿貿然 & mau6mau6jin4 & $\mathrm{CM}$ & "rashly; hastily" & 你點可以貿貿然應承任駕？ \\
\hline 342 & 微微 & mei4mei2 & $\mathrm{CM}$ & "slightly" & 佢微微有咗郁隻手。 \\
\hline 343 & 末 & mei6 & $\mathrm{CM}$ & "not yet" & 我未去。 \\
\hline 344 & 未必 & mei6bit1 & $\mathrm{CM}$ & "not necessarily" & 佢未必知哩件事。 \\
\hline 345 & 未曾 & mei6cang4 & $\mathrm{CM}$ & "never" & 我之前未曾搵過佢。 \\
\hline 346 & 未有耐 & mei6jau5noil & $\mathrm{CS}$ & $\begin{array}{l}\text { "not (in time)" } \\
\text { "not (in degree)" }\end{array}$ & $\begin{array}{l}\text { 我未有耐畢業。 } \\
\text { 你未有耐及得任。 }\end{array}$ \\
\hline 347 & 未免 & mei6min 5 & $\mathrm{CM}$ & "a bit too" & 佢政做未急太過份。 \\
\hline 348 & 未嘗 & mei6soeng 4 & $\mathrm{CM}$ & $\begin{array}{l}\text { "not (negative } \\
\text { sentence)" }\end{array}$ & 佢未嘗唔係一個好人。 \\
\hline 349 & 面對面 & $\min 6$ deoi $3 \min 6$ & CS & "face-to-face" & 我哋面對面坐响度。 \\
\hline 350 & 明明 & ming4ming 4 & $\mathrm{CM}$ & "certainly" & 明明係你做, 仲唔認。 \\
\hline 351 & 無情情 & mou4cing 4 cing 4 & CS & "without reason" & 你做也無情情鬧人唧？ \\
\hline 352 & 無從 & mou4cung 4 & $\mathrm{CM}$ & $\begin{array}{l}\text { "have no way of } \\
\text { doing something" }\end{array}$ & 呢件事我真係無從入手。 \\
\hline 353 & $\begin{array}{l}\text { 無端白事/ } \\
\text { 無情白事 } \\
\end{array}$ & $\begin{array}{l}\text { mou4dyun Ibaak6si6/ } \\
\text { mou4cing4baak6si6 }\end{array}$ & CS & "without reason" & 佢無端白事喊起上嚟。 \\
\hline 354 & 無端端 & mou4dyun 1 dyun I & CS & "without reason" & 我無端端俾人鬧。 \\
\hline 355 & 無非 & mou4feil & $\mathrm{CM}$ & $\begin{array}{l}\text { "nothing but; simply; } \\
\text { no more than; only" }\end{array}$ & $\begin{array}{l}\text { 佢講咁多嘢無非想你應承佢 } \\
\text { 嗜。 }\end{array}$ \\
\hline 356 & 無意中 & mou4ji3zungl & CS & $\begin{array}{l}\text { "not intentionally; by } \\
\text { chance" }\end{array}$ & 我無意中聽到佢話唔想嚟。 \\
\hline
\end{tabular}




\begin{tabular}{|c|c|c|c|c|c|}
\hline 357 & 無緣無故 & mou4jyun4mou4gu3 & $\mathrm{CM}$ & "without reason" & 你做也無緣無故唔返學？ \\
\hline 358 & 無辳䢃 & mou4laallaal & CS & "without reason" & 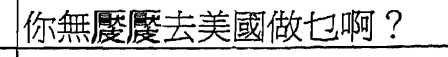 \\
\hline 359 & 無論如何 & mou4leon6jyu4ho4 & $\mathrm{CM}$ & $\begin{array}{l}\text { "in any case; at any } \\
\text { rate ; whatever } \\
\text { happens" }\end{array}$ & |你無論如何都要幫吓我啊！ \\
\hline 360 & 無時無刻 & mou4si4mou4hakI & $\mathrm{CM}$ & "all the time" & 我無時無刻都掛住佢。 \\
\hline 361 & 無 & mous & $\mathrm{CM}$ & $\begin{array}{l}\text { "have not" } \\
\text { "not (in fixed } \\
\text { phrases)" }\end{array}$ & $\begin{array}{l}\text { 我無去。 } \\
\text { 無緊要 ; 無有怕 }\end{array}$ \\
\hline 362 & 無得 & mou5dakl & CS & "it is not possible to" & 樓上嘈到我成晚無得剆。 \\
\hline 363 & 無幾何 & mou5gei2ho2 & $\mathrm{CS}$ & "rarely; seldom" & 我無幾何去睇戲。 \\
\hline 364 & 無日 & mou5jat6 & $\mathrm{CM}$ & $\begin{array}{l}\text { "all the time } \\
\text { (negative sentence)" }\end{array}$ & 我無日唔諗住佢。 \\
\hline 365 & 無さ & mou 5 mat1 & CS & "almost not" & 佢無さ出聲。 \\
\hline 366 & 無話 & mou5waa6 & $\mathrm{CS}$ & "never" & $\begin{array}{l}\text { 個衰仔由細到大無話俾啲錢 } \\
\text { 阿媽使個喎。 }\end{array}$ \\
\hline 367 & 每 & mui5 & $\mathrm{CM}$ & "whenever" & 我每見到佢一次就睤一次。 \\
\hline 368 & 難怪 & naan 4 gwaai3 & $\mathrm{CM}$ & "no wonder" & $\begin{array}{l}\text { 你成日都講大話, 難怪佢哋 } \\
\text { 唔信你。 }\end{array}$ \\
\hline 369 & 啱(啱)先 & ngaaml(ngaam1) sin 1 & $\mathrm{CS}$ & "just" & 我哋啱(啱)先講緊佢。 \\
\hline 370 & 啱啱 & ngaam Ingaaml & CS & \begin{tabular}{|l} 
"exactly" \\
"just"
\end{tabular} & $\begin{array}{l}\text { 我同任訩蔇哩䂆啱啱相反。 } \\
\text { 啱啱有人打電話俾你。 }\end{array}$ \\
\hline 371 & \begin{tabular}{|l|} 
啱啱好। \\
啱啱線
\end{tabular} & $\begin{array}{l}\text { ngaam1ngaam1hou2/ } \\
\text { ngaam 1ngaam1 } \sin 3\end{array}$ & CS & "just right" & 哩度啱啱好擺得落個雪檟。 \\
\hline 372 & 眼白白 & ngaan5baak6baak6 & CS & "helplessly" & 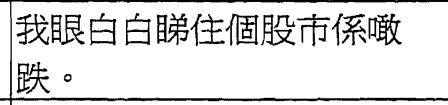 \\
\hline 373 & 硬係 & ngaang2hai6 & $\mathrm{CS}$ & $\begin{array}{l}\text { "in contrary to } \\
\text { speaker's will" }\end{array}$ & 㧽本書硬係買唔到挢。 \\
\hline 374 & 硬 & ngaang6 & $\mathrm{CS}$ & "certainly" & 輸硬；掂硬 \\
\hline 375 & 毅然 & ngai6jin4 & $\mathrm{CM}$ & "determinedly" & $\begin{array}{l}\text { 佢毅然放棄去美國讀畫蔇機 } \\
\text { 會。 }\end{array}$ \\
\hline 376 & 哽 & ngang3 & $\mathrm{CS}$ & "even very" & 哽複雜嘅理纾任都搞得掂。 \\
\hline 377 & 惡 & ngok3 & CS & $\begin{array}{l}\text { "with difficultly" } \\
\text { "painstakingly" } \\
\end{array}$ & $\begin{array}{l}\text { 條路好惡行。 } \\
\text { 我最近惡補英文。 }\end{array}$ \\
\hline 378 & 哩頭...咽頭... & niltau 4 ...go2tau $4 \ldots$ & $\mathrm{CS}$ & "as soon as" & $\begin{array}{l}\text { 我哩頭打緊電話搵佢, 嗰頭 } \\
\text { 佢就到。 }\end{array}$ \\
\hline 379 & 寧願 & ning4jyun2 & $\mathrm{CM}$ & "would rather" & 我寧願留响屋企。 \\
\hline 380 & \begin{tabular}{|l} 
而不耐/ \\
而唔耐
\end{tabular} & $\begin{array}{l}\text { noi6batInoi2/ } \\
\text { noi6m4noi2 } \\
\end{array}$ & CS & "once in a while" & 我耐不耐睇場戲。 \\
\hline 381 & 而唔中 & noi6m4zung1 & CS & "once in a while" & 我而唔中嚟下下。 \\
\hline 382 & 怕(且) & paa3(ce2) & $\mathrm{CS}$ & "perhaps" & 間屋怕(且)住得七八個人啩。 \\
\hline 383 & 頻頻 & pan4pan4 & $\mathrm{CM}$ & "again and again" & 佢頻頻望過嚟。 \\
\hline 384 & 湄空 & pang4hung1 & $\mathrm{CM}$ & "without foundation" & $\begin{array}{l}\text { 呢归的野都係佢憑空諗出嚟 } \\
\text { 咖。 }\end{array}$ \\
\hline 385 & 偏 & pinl & $\mathrm{CM}$ & $\begin{array}{l}\text { "contrary to speaker's } \\
\text { will" }\end{array}$ & 唔俾佢去, 佢偏要去。 \\
\hline 386 & 偏偏 & pin1pin1 & $\mathrm{CM}$ & $\begin{array}{l}\text { "contrary to speaker's } \\
\text { will" }\end{array}$ & $\begin{array}{l}\text { 我叫伯陪我行街, 佢偏偏唔 } \\
\text { 得閒。 }\end{array}$ \\
\hline
\end{tabular}




\begin{tabular}{|c|c|c|c|c|c|}
\hline & & & & "just" & 你做也偏偏要問佢唧？ \\
\hline 387 & 三番四次 & saamIfaanIsei3ci3 & $\mathrm{CS}$ & "repeatedly" & 佢三番四次嚟搵過你嘫。 \\
\hline 388 & 三爬兩撥(噉) & saam1paa4loeng5but6(gam2) & CS & "very quickly" & $\begin{array}{l}\text { 佢哋三爬兩撥(噉)就做起晒 } \\
\text { 啲埋野。 }\end{array}$ \\
\hline 389 & 雴時間 & saap3si4gaan1 & $\mathrm{CM}$ & "in a twinkling" & 我霓時間記唔起佢係邊個。 \\
\hline 390 & 稍第 & saau 2 wai4 & $\mathrm{CM}$ & "slightly" & 間屋㮐爲細咗啲。 \\
\hline 391 & 勢 & sai3 & CS & "absolutely" & 勢估唔到；唔勰勢係假。 \\
\hline 392 & 誓死 & saibsei2 & $\mathrm{CM}$ & "firmly" & 我誓死唔做哩啲嘢。 \\
\hline 393 & $\begin{array}{l}\text { 甚至(無)/ } \\
\text { 甚至(乎) }\end{array}$ & 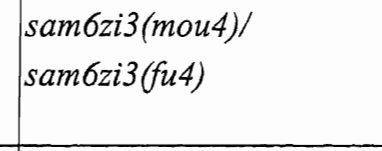 & $\mathrm{CM}$ & "even" & $\begin{array}{l}\text { 唔好話佢受咗少少傷, 甚至 } \\
\text { 無重傷、殘廢, 我都唔會離 } \\
\text { 開佢嘅。 }\end{array}$ \\
\hline 394 & 新 & $\operatorname{san} 1$ & $\mathrm{CM}$ & "newly" & 佢哋係新嚟厩。 \\
\hline 395 & 新近 & san1gan 6 & $\mathrm{CM}$ & "recently" & 佢係新近嚟呢度實習萔。 \\
\hline 396 & 晨早 & san4zou2 & CS & $\begin{array}{l}\text { "well in advance" } \\
\text { "early in the } \\
\text { morning" }\end{array}$ & $\begin{array}{l}\text { 我晨早搞㧨晒喇。 } \\
\text { 你晨(咁)早叫醒我做也？ }\end{array}$ \\
\hline 397 & 十分(之) & $\operatorname{sap} 6 f e n 1(z i 1)$ & $\mathrm{CM}$ & "very" & 今日我十分(之)勞氣。 \\
\hline 398 & 十成十 & sap6sing 4 sap6 & CS & "surely" & 佢噉嘅樣，十成十輸咗喇。 \\
\hline 399 & 失驚無神 & satlgeng1mou4san 4 & CS & "suddenly" & $\begin{array}{l}\text { 你失驚無神大叫一聲，想嚇 } \\
\text { 死人咩? }\end{array}$ \\
\hline 400 & 實 & sat6 & CS & $\begin{array}{l}\text { "actually" } \\
\text { "certainly" }\end{array}$ & $\begin{array}{l}\text { 話就話七百，我實收五百。 } \\
\text { 我實搵到佢。 }\end{array}$ \\
\hline 401 & 實梗 & sat6gang2 & CS & "certainly" & 你實梗唔嚟傌喇，係唔係？ \\
\hline 402 & 實係 & sat6hai6 & CS & "certainly" & 你咁高, 打波實係你贏喇。 \\
\hline 403 & 實行 & sat6hang 4 & CS & "certainly" & $\begin{array}{l}\text { 唔理你吡點諗, 我實行做落 } \\
\text { 去。 }\end{array}$ \\
\hline 404 & 實牙實齒 & sat6ngaa4sat6ci2 & CS & $\begin{array}{l}\text { "definitely; } \\
\text { undoubtedly" }\end{array}$ & 佢實牙實齒講過會嚟。 \\
\hline 405 & 實實在在 & sat6sat6zoi6zoi6 & CS & "in fact" & $\begin{array}{l}\text { 我實實在在唔想但咁擔心 } \\
\text { 我。 }\end{array}$ \\
\hline 406 & 實則 & sat6zakl & $\mathrm{CM}$ & "actually" & 佢實則唔係咁懶萔。 \\
\hline 407 & 實在 & sat6zoi6 & $\mathrm{CM}$ & "actually" & 我實在好唔捨得哩份工。 \\
\hline 408 & 首先 & $\operatorname{sau} 2 \sin 1$ & $\mathrm{CM}$ & "first of all" & 佢哋首先登記咗我哋個名。 \\
\hline 409 & 死 & sei2 & $\mathrm{CM}$ & $\begin{array}{l}\text { "desperately; } \\
\text { determinedly" }\end{array}$ & 死慳死抵; 佢死話人哋遲到。 \\
\hline 410 & 死(人)都 & sei2(jan4)doul & CS & $\begin{array}{l}\text { "in any case; } \\
\text { anyway" }\end{array}$ & \begin{tabular}{|l|} 
反正要俾錢，死人都要食番 \\
呴本。
\end{tabular} \\
\hline 411 & 死命(噉) & sei2meng6(gam2) & $\mathrm{CM}$ & "desperately" & 我吔死命(噉)跑。 \\
\hline 412 & 死死地氣 & sei2sei2dei6hei3 & $\mathrm{CS}$ & "have no way out" & 佢死死地氣返番屋企。 \\
\hline 413 & 成 & seng4 & CS & "full; as much as" & 哩本畫成二百蚊鴜。 \\
\hline 414 & 成日 & seng4jat6 & $\mathrm{CS}$ & "always" & 我成日嚟幫䘽哩間鋪頭。 \\
\hline 415 & 衰衰咃 & seoilseoildei2 & CS & "nevertheless" & $\begin{array}{l}\text { 佢衰裏哋都係你細佬，你要 } \\
\text { 睇實佢啊。 }\end{array}$ \\
\hline 416 & 純粹 & seon 4 seoi 5 & $\mathrm{CM}$ & "simply" & 佢純粹玩你咋。 \\
\hline 417 & 順便 & seon 6 bin 2 & $\mathrm{CM}$ & "conveniently" & $\begin{array}{l}\text { 你順便攞埋哩啲嘢返屋企 } \\
\text { 喇。 }\end{array}$ \\
\hline 418 & 順帶 & seon6daai3 & $\mathrm{CM}$ & "in passing" & $\begin{array}{l}\text { 我順帶提吓你哋，記住著校 } \\
\text { 服。 }\end{array}$ \\
\hline
\end{tabular}




\begin{tabular}{|c|c|c|c|c|c|}
\hline 419 & 順勢 & seon6sai3 & $\mathrm{CM}$ & $\begin{array}{l}\text { "by taking advantage } \\
\text { of an opportunity" }\end{array}$ & 我順勢打咗佢一杽。 \\
\hline 420 & 順手 & seon6sau2 & $\mathrm{CM}$ & "conveniently" & $\begin{array}{l}\text { 你出門口㧽陣時順手鎖埋道 } \\
\text { 門佢。 }\end{array}$ \\
\hline 421 & 私底下 & sildai2haa6 & $\mathrm{CS}$ & "in secret; in private" & 我哋已經私底下傾好咗。 \\
\hline 422 & 施施然 & $\operatorname{sil} \operatorname{siljin} 4$ & CS & $\begin{array}{l}\text { "leisurely; } \\
\text { unhurriedly" }\end{array}$ & $\begin{array}{l}\text { 過咗成個鐘, 佢先至施施然 } \\
\text { 黎到。 }\end{array}$ \\
\hline 423 & 私自 & silzi6 & $\mathrm{CM}$ & "secretly" & 佢私自改咗份約。 \\
\hline 424 & 試過 & si3gwo3 & CS & $\begin{array}{l}\text { "before; formerly; in } \\
\text { the past" }\end{array}$ & 哩個細路仔未試過咁靜。 \\
\hline 425 & 時...時... & si4...si4... & $\mathrm{CM}$ & $\begin{array}{l}\text { "sometimes...; } \\
\text { sometimes..." }\end{array}$ & 佢時喊時笑。 \\
\hline 426 & 時不時 & si4batlsi4 & $\mathrm{CM}$ & "sometimes" & 我時不時都會去喼吓弗。 \\
\hline 427 & 時時 & si4si4 & $\mathrm{CM}$ & "often; constantly" & 佢係外國時時掛住個仔。 \\
\hline 428 & 時時刻刻 & si4si4haklhakl & CS & "all the time" & 我時時刻刻都想同佢一齊。 \\
\hline 429 & 是必 & si6bit1 & CS & "certainly" & 你噉話佢，佢是必唔高興䅂。 \\
\hline 430 & 是但 & si6daan 6 & CS & $\begin{array}{l}\text { "randomly; as one } \\
\text { pleases" }\end{array}$ & 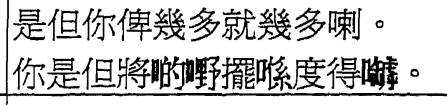 \\
\hline 431 & 事實上 & si6sat6soeng6 & $\mathrm{CM}$ & "in fact; actually" & 事實上有時真係唔係好明。 \\
\hline 432 & 食住個勢(就) & sik6zyu6go3sai3(zau6) & $\mathrm{CS}$ & $\begin{array}{l}\text { "by taking advantage } \\
\text { of a favourable } \\
\text { situation" }\end{array}$ & $\begin{array}{l}\text { 間公司食住個勢(就)推廣哩 } \\
\text { 隻產品。 }\end{array}$ \\
\hline 433 & 先(至) & $\sin 1(z i 3)$ & $\mathrm{CM}$ & $\begin{array}{l}\text { "first" } \\
\text { "then" } \\
\text { "as late as" } \\
\text { "just" } \\
\text { "expressing } \\
\text { emphasis" }\end{array}$ & $\begin{array}{l}\text { 邊件衫乾先就著邊件。 } \\
\text { 你勤力啲先(至)攞到好成績 } \\
\text { 榢嗎。 } \\
\text { 我聽日先(至)嚟。 } \\
\text { 而家先(至)一點鐘。 } \\
\text { 你先(至)係，我唔係！ }\end{array}$ \\
\hline 434 & 先後 & $\sin 1$ hau 6 & $\mathrm{CM}$ & "one after another" & $\begin{array}{l}\text { 我哋先後去咗北京同埋上海 } \\
\text { 玩。 }\end{array}$ \\
\hline 435 & 先先/先前 & $\sin 1 \sin 1 / \sin 1 \operatorname{cin} 4$ & CS & "before; previously" & $\begin{array}{l}\text { 我先先收咗大家五千蚊, 而 } \\
\text { 家用剩哩度啲錢, 回番水俾 } \\
\text { 你哋。 }\end{array}$ \\
\hline 436 & 擅自 & $\sin 6 z i 6$ & $\mathrm{CM}$ & $\begin{array}{l}\text { "without } \\
\text { authorisation" }\end{array}$ & 佢擅自改咗個計劃書。 \\
\hline 437 & 誠心 & sing 4 sam 1 & CS & "intentionally" & 佢誠心同我作對。 \\
\hline 438 & 少不免 & $\operatorname{siu} 2$ bat $1 \min 5$ & CS & "to a certain extent" & 你少不免會掛住佢。 \\
\hline 439 & 相當(之) & soengldongl(zil) & $\mathrm{CM}$ & $\begin{array}{l}\text { "quite; fairly; } \\
\text { considerably" }\end{array}$ & 份工要輪班, 相當(之)困身。 \\
\hline 440 & 上下 & soeng6haa2 & CS & "almost" & 佢嘅病上下好嘞。 \\
\hline 441 & 索性 & sok3sing 3 & $\mathrm{CM}$ & "simply" & 我索性自己一個人去行街。 \\
\hline 442 & 素來 & sou3loi4 & $\mathrm{CM}$ & "always; usually" & 我素來好準時, 唔會遲到。 \\
\hline 443 & 算 & syun 3 & $\mathrm{CM}$ & "fairly" & 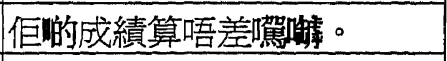 \\
\hline 444 & 太(過) & taai 3 (gwo3) & $\mathrm{CM}$ & "too" & 條禈太(過)窄, 著唔落。 \\
\hline 445 & 睇怕 & tai2paa3 & CS & $\begin{array}{l}\text { "apparently; } \\
\text { seemingly" }\end{array}$ & 天咁毉, 睇怕要落雨。 \\
\hline 446 & 偷 & taul & $\mathrm{CM}$ & "secretly" & 偷睇；偷聽 \\
\hline
\end{tabular}




\begin{tabular}{|c|c|c|c|c|c|}
\hline 447 & 偷雞 & taulgail & CS & "secretly" & 我係公司偷雞做自己嘢。 \\
\hline 448 & 偷偷吔 & taultauldei2 & CS & "secretly" & 佢偷偷吔出咗街。 \\
\hline 449 & 頭尾 & tau4mei5 & CS & $\begin{array}{l}\text { "from beginning to } \\
\text { end; altogether" }\end{array}$ & 我吡頭尾去咗五日。 \\
\hline 450 & 頭頭 & tau4tau2 & CS & "at the beginning" & 我哋僘頭頭得十幾個人。 \\
\hline 451 & 聽講(話) & tenglgong2(waab) & CS & "be told; hear of" & 聑愳(話)佢哋離咗婚。 \\
\hline 452 & 通常 & tung1soeng4 & $\mathrm{CM}$ & "usually" & $\begin{array}{l}\text { 我上網慨時候通常會聽快 } \\
\text { 歌。 }\end{array}$ \\
\hline 453 & 通通 & tungltung I & $\mathrm{CM}$ & "all; entirely" & 無用嘅嘢通通拎走。 \\
\hline 454 & 同埋 & tung4maai4 & CS & "together" & 我吔係同埋做嘢嘅工友。 \\
\hline 455 & 同時 & tung 4si4 & $\mathrm{CM}$ & "at the same time" & 佢哋同時衝過終點。 \\
\hline 456 & 話名 & waa6meng2 & CS & "nominally" & $\begin{array}{l}\text { 佢哋話名係親戚, 其實好少 } \\
\text { 來往。 }\end{array}$ \\
\hline 457 & 話晒 & waa6saai3 & CS & "after all" & 佢話晒都係你老豆。 \\
\hline 458 & 或者 & waak6ze2 & $\mathrm{CM}$ & "perhaps; maybe" & 你去勸佢, 佢或者會聽。 \\
\hline 459 & 玩吓手 & waan 2 haa 5 sau 2 & CS & "probably" & 你玩吓手唔捨得走掭啊。 \\
\hline 460 & 橫掂 & waang $4 \operatorname{dim} 6$ & CS & "anyway" & 橫掂都無用咯, 掉咗佢喇。 \\
\hline 461 & 唯獨是 & wai4duk6si6 & $\mathrm{CM}$ & "only" & 唯獨是有啲人唔肯做。 \\
\hline 462 & 唯有 & wai4jau5 & $\mathrm{CM}$ & "only; anyway" & 佢要等電話, 唯有唔出街住。 \\
\hline 463 & 永遠 & wing5jyun 5 & $\mathrm{CM}$ & "forever" & 我會永遠支持你嘅。 \\
\hline 464 & 互 & wu6 & $\mathrm{CM}$ & "each other" & 互篤；互扻 \\
\hline 465 & 互相 & wubsoengl & $\mathrm{CM}$ & $\begin{array}{l}\text { "mutually; each } \\
\text { other" }\end{array}$ & 我吔互相合作做埋啲鍒任。 \\
\hline 466 & 䁪听下眼 & zaam2haa5ngaan5 & CS & "in an instant" & 佢䀞印下眼唔知去咗邊。 \\
\hline 467 & 葓 & zaan2 & CS & $\begin{array}{l}\text { "in vain; for nothing" } \\
\text { "to an undesirable } \\
\text { result" }\end{array}$ & 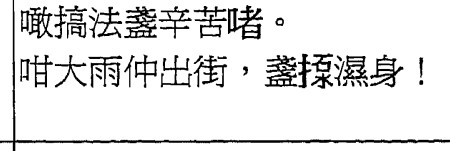 \\
\hline 468 & 掙啲 & zaangIdi1 & $\mathrm{CS}$ & "almost" & 我掙啲跌倒。 \\
\hline 469 & 枕長 & zam2coeng 4 & $\mathrm{CS}$ & "for a long time" & 佢枕長莭滁醫院度。 \\
\hline 470 & 枕住 & zam2zyu 6 & $\mathrm{CS}$ & "continuously" & 啲雨柁住係噉落。 \\
\hline 471 & 真係 & zan1hai6 & CS & "really" & 外便落雨無埞去, 真係悶親。 \\
\hline 472 & 周(不)時 & zau1(bat1)si4 & CS & "often; constantly" & 我周(不)時見到佢駕。 \\
\hline 473 & 就 & zau 6 & $\mathrm{CM}$ & $\begin{array}{l}\text { "very soon" } \\
\text { "as early as" } \\
\text { "as soon as" } \\
\text { "naturally (under } \\
\text { certain condition); } \\
\text { then" } \\
\text { "expressing contrast" } \\
\text { "expressing } \\
\text { concession (in } \\
\text { comparing two } \\
\text { things)" } \\
\text { "originally" } \\
\text { "only; merely" } \\
\text { "expressing } \\
\text { determination" }\end{array}$ & 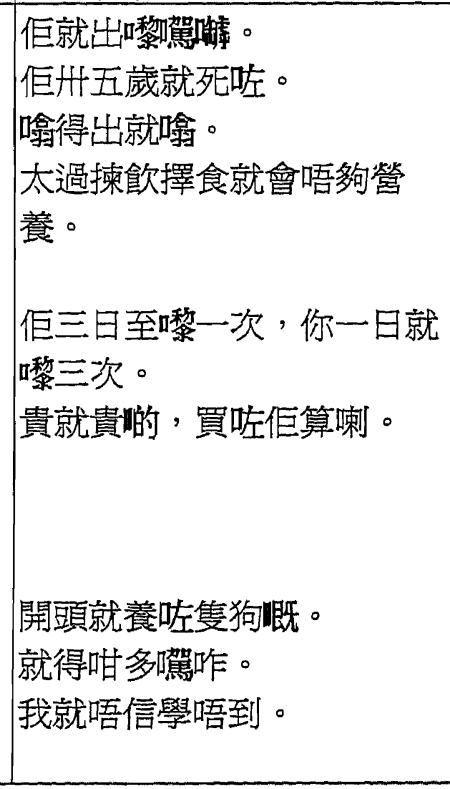 \\
\hline
\end{tabular}




\begin{tabular}{|c|c|c|c|c|c|}
\hline & & & & $\begin{array}{l}\text { "expressing } \\
\text { emphasis; the fact is } \\
\text { that" } \\
\text { "expressing contrary } \\
\text { belief" }\end{array}$ & $\begin{array}{l}\text { 就係佢囖。 } \\
\text { 佢唔係係哩度做嘢，吘嗰度 } \\
\text { 就真。 }\end{array}$ \\
\hline 474 & 就快 & zau6faai3 & $\mathrm{CS}$ & "very soon" & 啲飯就快者好。 \\
\hline 475 & 就吻 & zau6lai4 & $\mathrm{CS}$ & "very soon" & 佢就嚟搬屋。 \\
\hline 476 & 借啲意 & $z e 3 d i 1 j i 2$ & $\mathrm{CS}$ & $\begin{array}{l}\text { "by making an } \\
\text { excuse" }\end{array}$ & $\begin{array}{l}\text { 個會開咗一牛, 佢就借啲意 } \\
\text { 鬆人。 }\end{array}$ \\
\hline 477 & 借頭借路 & ze3tau4ze3lou6 & $\mathrm{CS}$ & $\begin{array}{l}\text { "by making an } \\
\text { excuse" }\end{array}$ & 佢成日借頭借路話唔得閒。 \\
\hline 478 & 正話 & zeng 3 waa 6 & CS & "just" & 佢正話都䌎呢度。 \\
\hline 479 & 最 & zeoi3 & $\mathrm{CM}$ & "the most" & 呢束花最好睇。 \\
\hline 480 & 最多/至多 & zeoi3dol/zi3dol & $\mathrm{CM}$ & $\begin{array}{l}\text { "at the worst" } \\
\text { "at the most" }\end{array}$ & $\begin{array}{l}\text { 人工咁奀, 我最多唔做。 } \\
\text { 我吔最多等多但五分鐘。 }\end{array}$ \\
\hline 481 & 最好 & zeoi3hou2 & $\mathrm{CM}$ & "it is good to" & 咁凍最好留係屋企。 \\
\hline 482 & 最少至少 & zeoi3siu2/zi3siu2 & $\mathrm{CM}$ & "at least" & 哩度最少坐三百人。 \\
\hline 483 & 盡 & zeon6 & $\mathrm{CM}$ & "exhaustively" & 盡寫；盡講 \\
\hline 484 & 盡情 & zeon6cing 4 & $\mathrm{CM}$ & $\begin{array}{l}\text { "as much as one } \\
\text { likes" }\end{array}$ & 我盡情唱 K。 \\
\hline 485 & 儘快 & zeon6faai3 & $\mathrm{CM}$ & "as fast as one could" & 你記得儘快交番返嫩。 \\
\hline 486 & 盡力 & zeon6lik6 & $\mathrm{CM}$ & "try one's best" & $\begin{array}{l}\text { 我吔盡力拉住苚樹，等伯唔 } \\
\text { 好秝。 }\end{array}$ \\
\hline 487 & 儘量 & zeon6loeng6 & $\mathrm{CM}$ & "to the full" & 你儘量食呋, 唔好客氣喎。 \\
\hline 488 & 儘早 & zeon6zou2 & $\mathrm{CM}$ & "as early as possible" & $\begin{array}{l}\text { 你儘早交埋篇論文咪可以去 } \\
\text { 玩籮。 }\end{array}$ \\
\hline 489 & 卒之 & zeotlzil & CS & "finally" & 卒之無賣俾佢。 \\
\hline 490 & 只不過 & zi2batlgwo 3 & $\mathrm{CS}$ & "merely; only; just" & 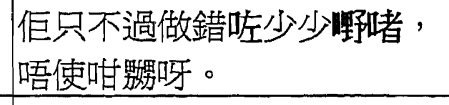 \\
\hline 491 & 只係 & zi2hai6 & $\mathrm{CS}$ & "merely; only; just" & 我只係去過大陸一次。 \\
\hline 492 & 至 & $z i 3$ & $\mathrm{CM}$ & $\begin{array}{l}\text { "the most" } \\
\text { "then" } \\
\text { "until" }\end{array}$ & $\begin{array}{l}\text { 我至憎人食煙。 } \\
\text { 你勤力啲至攞到好成績榢 } \\
\text { 嗎。 } \\
\text { 我聽日至嚟。 }\end{array}$ \\
\hline 493 & \begin{tabular}{|l} 
至多(咪)/ \\
至多(唔係)
\end{tabular} & $\begin{array}{l}\text { zi3dol(mai6)/ } \\
\text { zi3dol(m4hai6) }\end{array}$ & CS & "at the worst" & 我至多味瓄咗間屋佢囖。 \\
\hline 494 & 自不然 & zi6bat1jin4 & CS & "naturally" & \begin{tabular}{|l}
$\begin{array}{l}\text { 你呕吓下佢, 佢自不然唔會舅 } \\
\text { 你喇。 }\end{array}$ \\
\end{tabular} \\
\hline 495 & 自自然然 & zi6zi6jin4jin4 & $\mathrm{CS}$ & "naturally" & 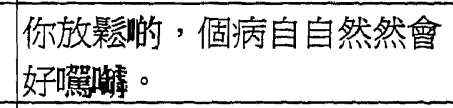 \\
\hline 496 & 即管 & ziklgun2 & $\mathrm{CS}$ & $\begin{array}{l}\text { "feel free to; not } \\
\text { hesitate to" }\end{array}$ & 你有七嘢即管講出洯。 \\
\hline 497 & 即刻 & ziklhaak1 & $\mathrm{CM}$ & "immediately" & 你等一陣，我即刻䝆。 \\
\hline 498 & 直 & $z i k 6$ & $\mathrm{CM}$ & "straightly; directly" & 直講；直去 \\
\hline 499 & 直情 & zik6cing4 & $\mathrm{CS}$ & $\begin{array}{l}\text { "straight; directly" } \\
\text { "simply; at all" }\end{array}$ & $\begin{array}{l}\text { 你直情話俾佢㯖我唔去咪得 } \\
\text { 囉。 } \\
\text { 咁少錢要招待咁多人直情考 } \\
\text { 起我吔！ }\end{array}$ \\
\hline
\end{tabular}




\begin{tabular}{|c|c|c|c|c|c|}
\hline & & & & "surely" & 直情係佢嗦度嘈喧巴閉喇！ \\
\hline 500 & 直頭 & zık6tau4 & CS & $\begin{array}{l}\text { "straight; directly" } \\
\text { "simply; at all" } \\
\text { "surely" } \\
\end{array}$ & $\begin{array}{l}\text { 直頭行去佢屋企, 唔好喺度 } \\
\text { 等踩。 } \\
\text { 直頭唔理佢行咗去！ } \\
\text { 佢直頭冤枉喇。 } \\
\end{array}$ \\
\hline 501 & 漸漸 & zim6zim2 & $\mathrm{CM}$ & "gradually" & 啲雨漸漸細落嚟。 \\
\hline 502 & 整整吓 & zing2zing2haa2 & CS & "gradually" & $\begin{array}{l}\text { 佢去咗外國咁多年, 整整吓 } \\
\text { 連中文都唔識講。 }\end{array}$ \\
\hline 503 & 正(一) & zing3(jat1) & $\mathrm{CS}$ & "indeed; really" & 你正(一)衰人！ \\
\hline 504 & 正式 & zing3sik1 & $\mathrm{CS}$ & "indeed; really" & 呢匀正式係行衰運。 \\
\hline 505 & 淨 & zing6 & CS & "only" & 淨食飯, 唔食餤。 \\
\hline 506 & 靜雞雞 & zing6gailgail & CS & "quietly" & 佢靜雞雞㕰枱面攞埕啲錢。 \\
\hline 507 & 淨係 & zing6hai6 & CS & $\begin{array}{l}\text { "only" } \\
\text { "constantly" }\end{array}$ & $\begin{array}{l}\text { 屋企淨係得我一個人。 } \\
\text { 唔好淨係話人，你自己都有 } \\
\text { 錯。 }\end{array}$ \\
\hline 508 & 接住 & zip3zyu6 & CS & $\begin{array}{l}\text { "immediately } \\
\text { afterwards; in a } \\
\text { minute" }\end{array}$ & 你講完之後, 我接住講。 \\
\hline \begin{tabular}{|l|}
509 \\
\end{tabular} & 照計 & ziu3gai3 & CS & $\begin{array}{l}\text { "according to reason; } \\
\text { in the ordinary } \\
\text { course of events; } \\
\text { normally" }\end{array}$ & 照計經理無理由唔敬格。 \\
\hline 510 & 照舊(噉)/照 & ziu3gau6(gam2)/ziu3 & $\mathrm{CM}$ & "as usual" & 我照舊(噉)去佢屋企玩。 \\
\hline 511 & 照樣(噉)/照 & ziu3joeng2(gam2)/ziu3 & $\mathrm{CM}$ & $\begin{array}{l}\text { "in the same old } \\
\text { way" }\end{array}$ & 我照樣(噉)幫佢打字。 \\
\hline 512 & 照例 & ziu3lai6 & $\mathrm{CM}$ & "as a rule" & 我哋照例六點鐘收鋪。 \\
\hline 513 & 照理 & ziu3lei5 & $\mathrm{CM}$ & "generally" & 佢照理應該俾你放假梠？ \\
\hline 514 & 照常 & ziu3soeng4 & $\mathrm{CM}$ & "as usual" & $\begin{array}{l}\text { 星期日我都照常返公司做 } \\
\text { 野。 }\end{array}$ \\
\hline 515 & 座底 & zo6dai2 & $\mathrm{CS}$ & "at least" & 哩度座底三百人。 \\
\hline 516 & 將近 & zoenglgan6 & $\mathrm{CM}$ & $\begin{array}{l}\text { "nearly; almost" } \\
\text { "very soon" } \\
\end{array}$ & $\begin{array}{l}\text { 而家將近八點。 } \\
\text { 飛機將近起飛踿。 }\end{array}$ \\
\hline 517 & 再 & $z o i 3$ & $\mathrm{CM}$ & $\begin{array}{l}\text { "again" } \\
\text { "more" } \\
\text { "continuously" } \\
\text { "afterwards" } \\
\text { "also" } \\
\end{array}$ & 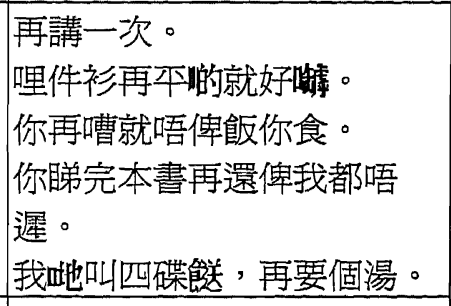 \\
\hline 518 & 再三 & zoi3saaml & $\mathrm{CM}$ & $\begin{array}{l}\text { "over and over } \\
\text { again" }\end{array}$ & 佢再三求我幫佢。 \\
\hline 519 & 再試 & zoi3si3 & CS & "again" & $\begin{array}{l}\text { 你再試係做呢, 我就唔睬 } \\
\text { 你! }\end{array}$ \\
\hline 520 & 撞啱 & zong6ngaam1 & CS & "by chance" & 撞啱佢唔係屋企。 \\
\hline 521 & 早日 & zou2jat6 & $\mathrm{CM}$ & "soon" & 希望你早日返嚟香港喇。 \\
\hline 522 & (老)早就 & (lou5)zou2zau6 & $\mathrm{CS}$ & "in advance" & $\begin{array}{l}\text { 你同我定, 我(老)早就準備好 } \\
\text { 晒。 }\end{array}$ \\
\hline 523 & 早知 & zou2zil & $\mathrm{CS}$ & $\begin{array}{l}\text { "having known this } \\
\text { beforehand" }\end{array}$ & 我早知唔去喇。 \\
\hline
\end{tabular}




\begin{tabular}{|c|c|c|c|c|c|}
\hline 524 & 早早 & zou 2 zou 2 & $\mathrm{CM}$ & "well in advance" & 我早早就嚟到呢度喇。 \\
\hline 525 & 足以 & $z u k l j i 5$ & $\mathrm{CM}$ & "enough" & $\begin{array}{l}\text { 你嗷講都唔足以證明佢無辜 } \\
\text { 䜖。 }\end{array}$ \\
\hline 526 & 足足 & zuklzukl & $\mathrm{CM}$ & "full; as much as" & 我足足等咗你牛個鐘。 \\
\hline 527 & 逐啲 & zuk6di1 & CS & "one by one" & 唔好心急, 逐的做。 \\
\hline 528 & 逐個 & zuk6go3 & $\mathrm{CM}$ & "one by one" & 佢逐個教啲細路哥寫字。 \\
\hline 529 & 逐一 & zuk6jat1 & $\mathrm{CM}$ & "one by one" & 佢逐一點名。 \\
\hline 530 & 終於 & zungljyul & $\mathrm{CM}$ & "finally" & 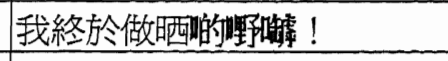 \\
\hline 531 & 終須 & zunglseoil & CS & $\begin{array}{l}\text { "anyhow; eventually; } \\
\text { after all" }\end{array}$ & 做落去終須有日唔掂。 \\
\hline 532 & 總共 & zung2gung 6 & $\mathrm{CM}$ & "altogether" & $\begin{array}{l}\text { 聖誕節褦住新年, 總共有十 } \\
\text { 日假。 }\end{array}$ \\
\hline 533 & 總係 & zung2hai6 & CS & "always; invariably" & 問親佢, 佢總係話唔知。 \\
\hline 534 & 總算 & zung2syun 3 & $\mathrm{CM}$ & $\begin{array}{l}\text { "at long last; finally" } \\
\text { "all things } \\
\text { considered" }\end{array}$ & $\begin{array}{l}\text { 我總算無白㶳心機。 } \\
\text { 佢總算對我唔錯。 }\end{array}$ \\
\hline 535 & 仲 & zung6 & CS & \begin{tabular}{|l} 
"more; even more" \\
"still; yet" \\
"additionally" \\
"expressing \\
emphasis" \\
\end{tabular} & $\begin{array}{l}\text { 今日仲凍過琴日。 } \\
\text { 佢仲未肯走。 } \\
\text { 佢日頭做嘢, 夜晚仲要讀書。 } \\
\text { 最初佢話試機呢, 我仲以瓜 } \\
\text { 佢會教晒啲 function 點用添。 }\end{array}$ \\
\hline 536 & 仲係 & zung6haib & CS & "still; nevertheless" & 十年無見, 你仲係咁後生。 \\
\hline 537 & 仲加 & zung6gaal & $\mathrm{CS}$ & "even more" & 聽日仲加辛苦眎啊。 \\
\hline 538 & 專 & zyun 1 & $\mathrm{CM}$ & "specially; always" & 佢專講大話。 \\
\hline 539 & 專程 & zyunl cing 4 & $\mathrm{CM}$ & "specially" & 我專程嚟探你嚜。 \\
\hline 540 & 專登 & zyunldangl & $\mathrm{CS}$ & $\begin{array}{l}\text { "for a special } \\
\text { purpose; specially" }\end{array}$ & 我專登嚟探你騭。 \\
\hline 541 & 專門 & zyun Imun2 & $\mathrm{CM}$ & "specially; always" & 佢專門整礐人。 \\
\hline 542 & 轉吓眼 & zyun2haa 5ngaan 5 & CS & "in an instant" & 佢轉叮眼就唔見人。 \\
\hline 543 & 轉(個)頭 & zyun3(go3)tau4 & CS & "later" & 我轉(個)頭敕搵你。 \\
\hline 544 & 絕對 & zyut6deoi3 & $\mathrm{CM}$ & "absolutely" & 啲消息絕對可靠騭！ \\
\hline
\end{tabular}

Number of Cantonese adverbs having cognates in Mandarin (CM): 242

Number of Cantonese-specific adverbs (CS): 302 


\section{Appendix 2: \\ Classification of Adverbs by Grammatical Meanings}

\section{Conjunctive Adverb}

\begin{tabular}{|c|c|c|}
\hline 不特止...仲... & bat ldak6zi2 ...zung6... & "not only...but also..." \\
\hline 固然之...只不過... & gu3jin4zi1 ...zi2bat1gwo $3 \ldots$ & "no doubt... only..." \\
\hline 一...就... & jatl ...zau6... & "as soon as... then..." \\
\hline 一旦...就... & jatldaan6...zau $6 \ldots$ & "once; in case... then..." \\
\hline 又 $_{\mathrm{a}} \ldots$ 又 $_{\mathrm{a}} \ldots$ & jau6...jau6... & "at the same time" \\
\hline 唔...又 X... & $m 4 \ldots$..jau6... & "not...but..." \\
\hline 咪 & mai6 & "then" \\
\hline 哩頭...调頭 ... & niltau 4 ...go 2 tau 4 .. & "as soon as" \\
\hline 先 ${ }_{\mathrm{a}}$ (至) & $\sin 1(z i 3)$ & "then" \\
\hline 就 a & zau 6 & "as soon as" \\
\hline 就 b & $z a u 6$ & "naturally (under certain condition); then" \\
\hline 就 c & zau6 & "expressing contrast" \\
\hline 就 d & zau 6 & $\begin{array}{l}\text { "expressing concession (in comparing two } \\
\text { things)" }\end{array}$ \\
\hline 至 & $z i 3$ & "then" \\
\hline
\end{tabular}

\section{Degree Adverb}

\begin{tabular}{|c|c|c|}
\hline 比較(上) & bei2gaau3(soeng6) & "fairly; rather" \\
\hline 牛 & bun 3 & "half" \\
\hline 超 & ciul & "super" \\
\hline 大大 & daai6daai6 & "greatly; enormously" \\
\hline 得滯 & daklzai6 & "too" \\
\hline 特別 & dak6bit6 & "particularly" \\
\hline 分外 & fan6ngoi6 & "particularly" \\
\hline 非常(之) & feilsoeng4(zil) & "very" \\
\hline 較爲 & gaau3wai4 & "relatively" \\
\hline 咁(鬼) & $\operatorname{gam} 3$ (gwai2) & "very" \\
\hline 近乎 & $\operatorname{gan} 6 f u 4$ & "close to" \\
\hline 更加 & gang3gaal & "even more" \\
\hline 幾a & gei2 & "rather" \\
\hline 幾 $\mathrm{b}$ & gei2 & "very" \\
\hline 幾咁/幾鬼 & gei2gam3/gei2gwai2 & "very" \\
\hline 極 $\mathrm{a}$ & gik6 & "extremely" \\
\hline 極之 & gik6zil & "extremely" \\
\hline 勁 & ging6 & "extremely" \\
\hline 鬼咁 & gwai2gam 3 & "very" \\
\hline
\end{tabular}




\begin{tabular}{|c|c|c|}
\hline 過分 & gwo3fan 6 & "excessively" \\
\hline 過頭 & gwo 3 tau 4 & "very; too" \\
\hline 輕輕 & heng6heng1 & "slightly" \\
\hline 好(鬼) & hou2(gwai2) & "extremely" \\
\hline 有啲 & jau5di1 & "a little bit" \\
\hline 異常 & ji6soeng 4 & "extremely" \\
\hline 認真 & jing2zan 1 & "absolutely" \\
\hline 完全 & jyun 4cyun4 & "completely" \\
\hline 遠遠 & jyun5jyun5 & "largely" \\
\hline 越...越... & jyut6.. jyut $6 \ldots$ & "the more...the more..." \\
\hline 越嚟越 & jyut6lai4jyut6 & "more and more" \\
\hline 靈舍 & ling 4 se 3 & "particularly" \\
\hline 略略 & loek6loek2 & "a little; a bit; slightly" \\
\hline 略篇 & loek6wai4 & "slightly" \\
\hline 萬二分 & maan6jibfan 1 & "very much; extremely" \\
\hline 微微 & mei4mei2 & "slightly" \\
\hline 哽 & ngang3 & "even very" \\
\hline 稍爲 & saau2wai4 & "slightly" \\
\hline 十分(之) & sap6fenl(ziI) & "very" \\
\hline 相當(之) & soengldongl(zil) & "quite; fairly; considerably" \\
\hline 算 & $\operatorname{syun} 3$ & "fairly" \\
\hline 太(過) & taai3(gwo3) & "too" \\
\hline 最 & zeoi3 & "the most" \\
\hline 儘量 & zeon6loeng6 & "to the full" \\
\hline 至b & $z i 3$ & "the most" \\
\hline 再a & zoi3 & "more" \\
\hline 仲 & zung6 & "more; even more" \\
\hline 仲加 & zung6gaal & "even more" \\
\hline
\end{tabular}

\section{Manner Adverb}

Manner

\begin{tabular}{|l|l|l|}
\hline 擺明 & baai2ming4 & "clearly" \\
\hline 百般 & baak3bun1 & "by every means" \\
\hline 白 & baak6 & "in vain; for nothing" \\
\hline 白白 & baak6baak6 & "in vain; to no purpose" \\
\hline 哇嘭㿦 & baang4baang2seng1 & "very quickly; at once" \\
\hline 齊齊 & cai4cai4 & "together" \\
\hline 親口 & canIhau2 & "(speak) personally" \\
\hline 親耳 & can1ji5 & "(hear) personally" \\
\hline 親眼 & canIngaan5 & "(see) personally" \\
\hline 親手 & can1sau2 & "with one's own hands" \\
\hline 親自 & canlzi6 & "in person" \\
\hline 趁早 & can3zou2 & "as early as possible" \\
\hline 脆脆哋 & ceoi3ceoi3dei2 & "reluctantly; grudgingly" \\
\hline 隨口 & ceoi4hau2 & "(speak) thoughtlessly" \\
\hline 隨手 & ceoi4sau2 & "conveniently" \\
\hline
\end{tabular}




\begin{tabular}{|c|c|c|}
\hline 速速 & cuklcukl & "very quickly; at once" \\
\hline 從中 & cung4zung1 & "from among" \\
\hline 打掂 & daa2dim6 & "vertically" \\
\hline 打成 & daa2dung 6 & "vertically" \\
\hline 打孙 & daa2maal & "in pair" \\
\hline 打橫 & daa2waang 4 & "horizontally" \\
\hline 大大步 & daai6daai6bou6 & "with wide steps" \\
\hline 大大啖 & daai6daai6daam6 & "at a big mouthful" \\
\hline 大大力 & daai6daai6lik6 & "with a strong force" \\
\hline 大大聲 & daai6daai6seng1 & "very loudly" \\
\hline 大啖大啖 & daai6daam6daai6daam6 & "at a big mouthful" \\
\hline 大力 & daai6lik6 & "vigorously" \\
\hline 單獨 & daanlduk6 & "alone" \\
\hline 特登 & dak6dangl & "for a special purpose; specially" \\
\hline 突然(之)間 & dat6jin4(zil)gaan1 & "suddenly" \\
\hline 氹氹师緙 & dam4dam4kwaakl & "in a circle" \\
\hline 叠埋心水 & dip6maai4sam1seoi2 & "wholeheartedly; attentively" \\
\hline 當面 & dong1min 2 & "in somebody's presence" \\
\hline 當眺 & donglzung 3 & "in the presence of all" \\
\hline 快啲 & faai3dil & "quickly" \\
\hline 快快脆脆 & faai3faai3ceoi3ceoi3 & "quickly" \\
\hline 快手快腳 & faai3sau2faai3goek3 & "quickly" \\
\hline 分頭 & fanltau4 & "separately" \\
\hline 忽然(之)間 & fatljin4(zil)gaan1 & "suddenly" \\
\hline 監粗 & gaam 3 coul & "unyieldingly" \\
\hline 監生 & gaam 3 saang 1 & "while still alive" \\
\hline 夾硬 & gaap3ngaang2 & "unyieldingly" \\
\hline 夾手夾腳 & gaap3sau 2gaap 3 goek 3 & "jointly" \\
\hline 雞噉腳 & gailgam 2 goek3 & "very quickly" \\
\hline 急急 & gap lgap1 & "nearly at once" \\
\hline 急急腳 & gaplgap Igoek3 & "at a fast pace" \\
\hline 急急忙忙 & gaplgap 1mong4mong4 & "in a hurry; in a haste" \\
\hline 急住 & gaplzyu6 & "in a hurry; in a haste" \\
\hline 極力 & gik6lik6 & "do one's utmost" \\
\hline 各自各 & gok3(zi3)gok3 & "respectively" \\
\hline 乾 & gon1 & "in a dry manner" \\
\hline 公然 & gungljin 4 & "openly" \\
\hline 滾水淥腳(噉) & gwan2seoi2luk6goek3(gam2) & "in a hurry" \\
\hline 閒閒哋 & haan4haan2dei2 & "very easily" \\
\hline 係都 & hai6doul & "with insistence" \\
\hline 係噉意 & haibgam $2 j i 2$ & "perfunctorily" \\
\hline 刻意 & haklji3 & "painstakingly" \\
\hline 口快快(噉) & hau2faai3faai3(gam2) & "without hesitation" \\
\hline 好好(哋) & hau2hau2(dei2) & "well" \\
\hline 口口聲聲 & hau 2 hau 2 seng 1 sengl & "(say) again and again" \\
\hline 起勢 & hei2sai3 & "desperately" \\
\hline 輕易 & hinglji6 & "rashly" \\
\hline 開口埋口 & hoilhau2maai4hau2 & "(keep) talking over the same thing \\
\hline
\end{tabular}




\begin{tabular}{|c|c|c|}
\hline 好意思 & hou2ji3sil & "have the nerve" \\
\hline 一步步 & jat1bou2bou6 & "step by step; progressively" \\
\hline 一齊 $\mathrm{a}$ & jatlcai4 & "together" \\
\hline 一口價 & jatlhau2gaa3 & "at a fixed price" \\
\hline 一口氣 & jatl hau2hei3 & "in one breath; without a break" \\
\hline 二- & jatljat1 & "one by one" \\
\hline 一輪嘴 & jat1leon2zeoi2 & "without a break" \\
\hline 一連 & jat1lin4 & "in succession" \\
\hline 一眼 & jatlngaan 5 & "in a glimpse" \\
\hline 一心 & jatlsam1 & "wholeheartedly" \\
\hline 一心 $b$ & jatlsaml & "intentionally" \\
\hline 一手 & jat1sau2 & "single-headed" \\
\hline 一手一腳 & jat1sau2jatlgoek3 & "by oneself" \\
\hline 由頭 & jau4tau4 & "from the beginning" \\
\hline 有意 & jau5ji3 & "intentionally; deliberately" \\
\hline 依稀 & jilheil & "vaguely" \\
\hline 依時(依候) & jilsi4(jilhau6) & "punctually" \\
\hline 現皃淣 & jin6dauldau1 & "in cash" \\
\hline 卡那(卡那) & kaa3laa1 (kaa3laal) & "alternatively" \\
\hline 擒擒青 & kam4kam2ceng 1 & "quickly" \\
\hline 及早 & kap6zou2 & "as soon as possible" \\
\hline 狂 & kwong4 & "crazily" \\
\hline 嗱(嗱)臨 & laa4(laa4)lam4 & "very quickly; at once" \\
\hline 嗱嗱聲 & laa4laa2seng1 & "very quickly; at once" \\
\hline 臨急臨忙 & lam4gap Ilam4mong4 & "at the last moment" \\
\hline 臨時臨急 & lam4si4lam4gap1 & "at the last moment" \\
\hline 笠亂 & lap6lyun2 & "carelessly; casually; at random" \\
\hline 輪流 & leon4lau2 & "in turn" \\
\hline 哩哩啦啦 & Zi4li1laa4laa4 & "very quickly" \\
\hline 力 & lik6 & "make every effort" \\
\hline 鏈住條頸 & Iin2zyu6tiu4geng2 & "unwillingly" \\
\hline 連氣 & Iin4hei3 & "in succession" \\
\hline 連續 & $\operatorname{lin} 4 z u k 6$ & "successively" \\
\hline 兩份 & loeng5fan2 & "together" \\
\hline 來回 & loi4wui4 & "back and forth" \\
\hline 落手 & lok6sau2 & "by oneself; in person" \\
\hline 落手落腳 & lok6sau2lok6goek3 & "by oneself; in person" \\
\hline 陸陸續續 & luk6luk6zuk6zuk6 & "in succession" \\
\hline 陸續 & luk6zuk6 & "in succession" \\
\hline 亂(咁) & lyun2(gam 3$)$ & "thoughtlessly" \\
\hline 唔經唔覺 & $m 4$ ging $1 \mathrm{~m} 4$ gok 3 & "without knowing consciously" \\
\hline 唔聲唔聲 & $m 4$ seng $1 m 4$ seng 1 & "quietly" \\
\hline 誤 & $m 6$ & "mistakenly" \\
\hline 漫 & maan6 & "casually" \\
\hline 慢慢 & maan6maan2 & "slowly" \\
\hline 猛(咁) & maang5(gam 3) & "desperately" \\
\hline 貿然 & mau6jin4 & "rashly; hastily" \\
\hline 貿貿然 & mau6mau6jin4 & "rashly; hastily" \\
\hline
\end{tabular}




\begin{tabular}{|c|c|c|}
\hline 面對面 & $\min 6$ deoi3min 6 & "face-to-face" \\
\hline 無情情 & mou4cing 4 cing 4 & "without reason" \\
\hline \begin{tabular}{|l} 
無從 \\
\end{tabular} & mou4cung 4 & "have no way of doing something" \\
\hline \begin{tabular}{|l|} 
無端白事/ \\
無情白事
\end{tabular} & $\begin{array}{l}\text { mou4dyun1baak6si4/ } \\
\text { mou4cing4baak6si6 }\end{array}$ & "without reason" \\
\hline \begin{tabular}{|l|l} 
無端端 \\
\end{tabular} & mou4dyun1dyun1 & "without reason" \\
\hline \begin{tabular}{|l|} 
無意中 \\
\end{tabular} & mou4ji3zungl & "not intentionally; by chance" \\
\hline 無緣無故 & mou4jyun4mou4gu3 & "without reason" \\
\hline 無檚厴 & mou4laallaal & "without reason" \\
\hline 啱啱 a & ngaam Ingaaml & "exactly" \\
\hline \begin{tabular}{|l|} 
啱啱好/ \\
嵱啱線
\end{tabular} & $\begin{array}{l}\text { ngaam1ngaam1hou2/ } \\
\text { ngaam1ngaam1sin } 3\end{array}$ & "just right" \\
\hline 眼白白 & ngaan5baak6baak6 & "helplessly" \\
\hline 毅然 & ngai6jin4 & "determinedly" \\
\hline 惡 & ngok3 & "with difficultly" \\
\hline 惡 & ngok3 & "painstakingly" \\
\hline 憑空 & pang4hung1 & "without foundation" \\
\hline 三爬兩撥(噉) & saam1paa4loeng5but6(gam2) & "very quickly" \\
\hline 誓死 & sai6sei2 & "firmly" \\
\hline 新 & $\operatorname{san} 1$ & "newly" \\
\hline 失警無神 & satlgeng1mou $4 \operatorname{san} 4$ & "suddenly" \\
\hline 死 & sei2 & "desperately; determinedly" \\
\hline 死命(噉) & sei2meng6(gam2) & "desperately" \\
\hline 死死地氣 & sei2sei2dei6hei3 & "have no way out" \\
\hline 順便 & $\operatorname{seon} 6$ bin 2 & "conveniently" \\
\hline 順帶 & seon6daai3 & "in passing" \\
\hline 順勢 & seon6sai3 & "by taking advantage of an opportunity" \\
\hline 順手 & seon6sau2 & "conveniently" \\
\hline 私底下 & sildai2haa6 & "in secret; in private" \\
\hline 施施然 & silsiljin4 & "leisurely; unhurriedly" \\
\hline 私自 & silzi6 & "secretly" \\
\hline 是但 & si6daan 6 & "randomly; as one pleases" \\
\hline 食住個勢(就) & sik6zyu6go3sai3(zau6) & $\begin{array}{l}\text { "by taking advantage of a favourable } \\
\text { situation" }\end{array}$ \\
\hline 先後 & $\sin 1$ hau6 & "one after another" \\
\hline 擅自 & $\sin 6 z i 6$ & "without authorisation" \\
\hline 誠心 & sing 4 sam 1 & "intentionally" \\
\hline 偷 & taul & "secretly" \\
\hline 偷雞 & taulgail & "secretly" \\
\hline 偷偷吔 & taul tauldei2 & "secretly" \\
\hline 同埋 & tung4maai4 & "together" \\
\hline 話名 & waa6meng2 & "nominally" \\
\hline 互 & wu6 & "each other" \\
\hline 互相 & wubsoengI & "mutually; each other" \\
\hline 盖 $\mathrm{a}$ & zaan2 & "in vain; for nothing" \\
\hline 借狛意 & ze3dilji2 & "by making an excuse" \\
\hline 借頭借路 & ze3tau4ze3lou6 & "by making an excuse" \\
\hline 盡 & zeon6 & "exhaustively" \\
\hline
\end{tabular}




\begin{tabular}{|l|l|l|}
\hline 盡情 & zeon6cing4 & "as much as one likes" \\
\hline 儘快 & zeon6faai3 & "as fast as one could" \\
\hline 盡力 & zeon6lik6 & "try one's best" \\
\hline 儘早 & zeon6zou2 & "as early as possible" \\
\hline 直 & zik6 & "straight; directly" \\
\hline 直情 a & zik6cing4 & "straight; directly" \\
\hline 直頭 a & zik6tau4 & "straight; directly" \\
\hline 漸漸 & zim6zim2 & "gradually" \\
\hline 整整吓 & zing2zing2haa2 & "gradually" \\
\hline 靜雞雞 & zing6gailgai1 & "quietly" \\
\hline 照舊(噉)/照 & ziu3gau6(gam2)/ziu3 & "as usual" \\
\hline 照樣(噉)/照 & ziu3joeng2(gam2)/ziu3 & "in the same old way" \\
\hline 照常 & ziu3soeng4 & "as usual" \\
\hline 足以 & zuk1ji5 & "enough" \\
\hline 逐啲 & zuk6di1 & "one by one" \\
\hline 逐個 & zuk6go3 & "one by one" \\
\hline 遂一 & zuk6jat1 & "one by one" \\
\hline 專 & zyun1 & "specially; always" \\
\hline 專程 & zyun1cing4 & "specially" \\
\hline 專登 & zyun1dang1 & "for a special purpose; specially" \\
\hline 專門 & zyun1mun2 & "specially; always" \\
\hline
\end{tabular}

Repetition

\begin{tabular}{|l|l|l|}
\hline 重 & cung4 & "again; once more" \\
\hline 重新 & cung4san1 & "again; anew" \\
\hline 從頭 & cung4tau4 & "anew; once again" \\
\hline 反複 & faan2fuk1 & "repeatedly" \\
\hline 極 $\mathrm{b}$ & gik6 & "repeatedly" \\
\hline 一次又一次 & jat1ci3jau6jat1ci3 & "time and again; repeatedly" \\
\hline 一再 & jat1zoi3 & "time and again" \\
\hline 又 & jau6 & "repeatedly; again" \\
\hline 又試 & jau6si3 & "again" \\
\hline 三番四次 & saam1faan1sei3ci3 & "repeatedly" \\
\hline 再 $b$ & zoi3 & "again" \\
\hline 再三 & zoi3saam1 & "over and over again" \\
\hline 再試 & zoi3si3 & "again" \\
\hline
\end{tabular}

\section{Modal Adverb}

Level of Doubt/Certainty i.e. Epistemic Condition on the Clause

\begin{tabular}{|l|l|l|}
\hline 包 & baau1 & "certainly" \\
\hline 似乎 & ciffu4 & "seemingly" \\
\hline 的(而且)確 & dik1(ji4ce2)kok3 & "really; indeed" \\
\hline 多多少少 & doldolsiu2siu2 & "to a certain extent" \\
\hline 多數 & do1sou3 & "probably; most likely" \\
\hline 斷(斷) & dyun6(dyun6) & "absolutely" \\
\hline
\end{tabular}




\begin{tabular}{|c|c|c|}
\hline 分明 & fan1ming4 & "clearly; obviously" \\
\hline 梗 & gang2(hai6) & "undoubtedly" \\
\hline 梗 $\mathrm{b}($ 係 $)$ & gang2(hai6) & "certainly" \\
\hline 九成 & gau2sing 4 & "very likely" \\
\hline 肯定 & hang2 ding 6 & "surely" \\
\hline 可能 & ho2nang4 & "probably" \\
\hline 好似 & hou 2 ci5 & "presumably" \\
\hline 一定 & jat1ding6 & "certainly; surely" \\
\hline 一口 & jat1hau2 & "with certainty" \\
\hline 有 & jau5 & "surely; already" \\
\hline 確係 & kok2hai6 & "really; indeed" \\
\hline 確實 & kok2sat6 & "really; indeed" \\
\hline 唔多唔少 & $m 4 d o \operatorname{lm} 4 \operatorname{siu} 2$ & "to a certain extent" \\
\hline 唔見得 & m4gin 3 dakl & "not likely" \\
\hline 唔使問 & m4sai2man6 & "undoubtedly" \\
\hline 唔使審 & $m 4$ sal2sam2 & "undoubtedly" \\
\hline 唔通 a & m4tung 1 & "can it be that; is it possible that" \\
\hline 唔知 a & $m 4 z i 1$ & "really" \\
\hline 咪 $\mathrm{b}$ & mai6 & "exactly; really" \\
\hline 未必 & mei6bit I & "not necessarily" \\
\hline 明明 & ming 4 ming 4 & "certainly" \\
\hline 硬 & ngaang6 & "certainly" \\
\hline 怕(且) & paa3(ce2) & "perhaps" \\
\hline 勢 & sai3 & "absolutely" \\
\hline 十成十 & sap6sing 4 sap6 & "surely" \\
\hline 實 & sat6 & "certainly" \\
\hline 實梗 & sat6gang2 & "certainly" \\
\hline 實係 & sat6haib & "certainly" \\
\hline 實行 & sat6hang4 & "certainly" \\
\hline 實牙實荗 & sat6ngaa4sat6ci2 & "definitely; undoubtedly" \\
\hline 是必 & si6bitl & "certainly" \\
\hline 少不免 & $\operatorname{siu} 2 b a t \min 5$ & "to a certain extent" \\
\hline 睇怕 & tai2paa3 & "apparently; seemingly" \\
\hline 或者 & waak6ze2 & "perhaps; maybe" \\
\hline 玩吓手 & waan 2 haa 5 sau 2 & "probably" \\
\hline 真係 & zanlhai6 & "really" \\
\hline 直情 $\mathrm{b}$ & zik6cing4 & "surely" \\
\hline 直頭 b & zik6tau4 & "surely" \\
\hline 正( & zing3(jat1) & "indeed; really" \\
\hline 正式 & zing3sikI & "indeed; really" \\
\hline 絕對 & zyut6deoi3 & "absolutely" \\
\hline
\end{tabular}

Speaker's Attitude

\begin{tabular}{|l|l|l|}
\hline 弊在 & bai6zoi6 & "unfortunately" \\
\hline 不妨 & bat1fong4 & "there is no harm in; might as well" \\
\hline 不如 & batljyu4 & "it would be better to" \\
\hline 不期然 & bat1kei4jin4 & "unexpectedly" \\
\hline
\end{tabular}




\begin{tabular}{|c|c|c|}
\hline 不愧 & bat1kwais & "be worthy of" \\
\hline 不知 & batlzil & "to speaker's/hearer's surprise" \\
\hline 查實 & caa4sat6 & "in fact; actually" \\
\hline 始終 & ci2zung1 & "after all" \\
\hline 千新 & cin1kei4 & "for heaven's sake" \\
\hline 情願 & cing4jyun 2 & "would rather" \\
\hline 點(不)知 & $\operatorname{dim} 2$ (bat1)zi1 & "unexpectedly" \\
\hline 當然 & dongljin4 & "of course" \\
\hline 都a & doul & "after all; rather" \\
\hline 都。 & doul & "expressing emphasis; already" \\
\hline 都係 & doulhai6 & "had better" \\
\hline 到底 & dou3dai2 & "actually; exactly" \\
\hline 反而 & faan $2 j i 4$ & "on the contrary" \\
\hline 反爲 & faan 2 wai4 & "on the contrary; instead" \\
\hline 反正 & faan2zing 3 & "anyway" \\
\hline 假假哋 & gaa2gaa2dei2 & "in any case; anyhow" \\
\hline 簡直 & gaan2zik6 & "simply" \\
\hline 咁啱 & gam3ngaam 1 & "by chance" \\
\hline 根本(上) & gan1bun2(soeng6) & "at all; simply" \\
\hline 夠 & gau3 & "as well; too" \\
\hline 究竟 & gau3ging2 & "actually; exactly" \\
\hline 幾大 & gei2daai2 & "no matter what, how, etc." \\
\hline 居然 & geoiljin4 & "unexpectedly" \\
\hline 竟然 & ging2jin4 & "unexpectedly" \\
\hline 乾脆 & gonlceoi3 & "simply" \\
\hline 姑且 & gulce2 & "for the time being" \\
\hline 果然 & gwo2jin4 & "accordingly" \\
\hline 何必 & ho4bit1 & "why bother" \\
\hline 何苦 & ho4fu2 & "why bother" \\
\hline 好好醜醜 & hou 2 hou 2 cau2cau2 & "in any case; whatever happens" \\
\hline 好唔好都 & hou $2 m 4$ hou 2 doul & "in any case; whatever happens" \\
\hline 好在 & hou2zoi6 & "fortunately" \\
\hline 一日 & jatljat6 & "in the final analysis; at bottom" \\
\hline 一於 & jatljyul & "regardless of the consequences" \\
\hline 一於 $\mathrm{b}$ & jatljyu1 & "simply; just; altogether" \\
\hline 一於 $\mathrm{c}$ & jatljyul & "expressing a decision" \\
\hline 有得 $a$ & jau 5 dakl & "it is possible to" \\
\hline 有得 $b$ & jau5dakl & "it is worthwhile to" \\
\hline 有理無理 & jau5lei5mou5lei5 & "regardless of the consequences" \\
\hline$\underline{Z}_{c}$ & jau6 & "but" \\
\hline $\bar{X}_{d}$ & jau6 & "expressing emphasis" \\
\hline 應份 & jinglfan 6 & "necessarily" \\
\hline 原來 & jyun4loi4 & "originally" \\
\hline 其實 & kei4sat6 & "actually; in fact" \\
\hline 唔怪(之)得 & m4gwaai3(zil)dakl & "that is why" \\
\hline 唔通 b & m4tungl & "expressing rhetorical question" \\
\hline 唔掙在 & m4zaanglzoi6 & "never mind" \\
\hline 唔知 $b$ & $m 4 z i 1$ & "unknowingly" \\
\hline
\end{tabular}




\begin{tabular}{|c|c|c|}
\hline 未免 & $\operatorname{mei6\operatorname {min}5}$ & "a bit too" \\
\hline 無非 & moutfeil & "nothing but; simply; no more than; only" \\
\hline 無論如何 & mou4leon6jyu4ho4 & "in any case; at any rate ; whatever happens" \\
\hline 難怪 & naan4gwaai3 & "no wonder" \\
\hline 硬係 & ngaang2hai6 & "in contrary to speaker's will" \\
\hline 寧願 & ning4jyun2 & "would rather" \\
\hline 偏 & $\operatorname{pin} 1$ & "contrary to speaker's will" \\
\hline 偏偏 a & pin1pin1 & "contrary to speaker's will" \\
\hline 甚至(無)/甚至(乎) & sam6zi3(mou4)/sam6zi3(fu4) & "even" \\
\hline 實 & sat6 & "actually" \\
\hline 實實在在 & sat6sat6zoi6zoi6 & "in fact" \\
\hline 實則 & sat6zak1 & "actually" \\
\hline 實在 & sat6zoi6 & "actually" \\
\hline 死(人)都 & sei2(jan4)doul & "in any case; anyway" \\
\hline 衰衰哋 & seoilseoildei2 & "nevertheless" \\
\hline 純粹 & seon 4 seoi 5 & "simply" \\
\hline 事實上 & sibsat6soeng6 & "in fact; actually" \\
\hline 先 $\mathrm{b}$ (至) & $\sin 1(z i 3)$ & "as late as" \\
\hline 先 $c$ (至) & $\sin 1(z i 3)$ & "expressing emphasis" \\
\hline 索性 & sok3sing 3 & "simply" \\
\hline 話晒 & waa6saai3 & "after all" \\
\hline 橫掂 & waang4dim 6 & "anyway" \\
\hline 唯有 & wai4jau 5 & "only; anyway" \\
\hline 䓝 b & zaan2 & "to an undesirable result" \\
\hline 就 e & zau6 & "as early as" \\
\hline 就 $\mathrm{f}$ & zau6 & "expressing determination" \\
\hline 就 $\mathrm{g}$ & zau6 & "expressing emphasis; the fact is that" \\
\hline 就 $\mathrm{h}$ & zau6 & "expressing contrary belief" \\
\hline 最多/至多a & zeoi3dol/zi3dol & "at the worst" \\
\hline 最好 & zeoi3hou2 & "it is good to" \\
\hline 至 $_{c}$ & $z i 3$ & "as late as" \\
\hline 至多(咪)/至多(唔係) & zi3dol(mai6)/zi3dol(m4hai6) & "at the worst" \\
\hline 自不然 & zi6batljin4 & "naturally" \\
\hline 自自然然 & zi6zi6jin4jin4 & "naturally" \\
\hline 即管 & ziklgun2 & "feel free to; not hesitate to" \\
\hline 直情 c & zik6cing4 & "simply; at all" \\
\hline 直頭 c & zik6tau4 & "simply; at all" \\
\hline 撞啱 & zong6ngaam 1 & "by chance" \\
\hline 早知 & zou2zi1 & "having known this beforehand" \\
\hline 仲 & zung6 & "expressing emphasis" \\
\hline
\end{tabular}

Speaker's Point of View

\begin{tabular}{|l|l|l|}
\hline 大體(上) & daai6tai2(soeng6) & "on the whole" \\
\hline 奉旨 & fung6zi2 & "as a rule" \\
\hline 計正 & gai3zeng3 & "under normal circumstances; as a rule" \\
\hline 基本上 & geilbun2soeng6 & "basically" \\
\hline 嗱埋 a & laa2maai4 & "generally speaking" \\
\hline
\end{tabular}




\begin{tabular}{|l|l|l|}
\hline 聽講(話) & teng1gong2(waa6) & "be told; hear of; by hearsay" \\
\hline 照計 & ziu3gai3 & $\begin{array}{l}\text { "according to reason; in the ordinary course of } \\
\text { events; normally" }\end{array}$ \\
\hline 照例 & ziu3lai6 & "as a rule" \\
\hline 照理 & ziu3lei5 & "generally" \\
\hline 總算 a & zung2syun3 & $\begin{array}{l}\text { "considering everything; all things } \\
\text { considered; on the whole" }\end{array}$ \\
\hline
\end{tabular}

\section{Negation Adverb}

\begin{tabular}{|c|c|c|}
\hline 幾時得嚟 $\mathrm{a}$ & gei2si4dak1lai4 & "not (within a period of time)" \\
\hline 幾時得嚟 b & gei2si4dak1lai4 & "not (comparatively speaking)" \\
\hline 鬼 & gwai2 & "not" \\
\hline 唔(鬼) & m4(gwai2) & "not" \\
\hline 唔多 & $m 4 d o 1$ & "not very" \\
\hline 唔慌 & $m 4$ fong 1 & "absolutely not" \\
\hline 唔好 & m4hou2 & "don't" \\
\hline 唔使 & $m 4$ sai2 & "not necessarily" \\
\hline 咪 & mai5 & "don't" \\
\hline 末 & mei6 & "not yet" \\
\hline 未曾 & mei6cang4 & "never" \\
\hline 未有耐 & mei6jau5noil & "not (in time)" \\
\hline 未有耐 b & mei6jau5noil & "not (in degree)" \\
\hline 未嘗 & mei6soeng4 & "not (negative sentence)" \\
\hline 無 & mou5 & "have not" \\
\hline 無 $\mathrm{b}$ & mous & "not (in fixed phrases)" \\
\hline 無得 & mou5dak1 & "it is not possible to" \\
\hline 無さ & mou5mat1 & "almost not" \\
\hline $\begin{array}{l}\text { 無話 } \\
\end{array}$ & mou 5 waa6 & "never" \\
\hline
\end{tabular}

\section{Scope Adverb}

\begin{tabular}{|l|l|l|}
\hline 差啲 & caaldi1 & "almost" \\
\hline 隨處 & ceoi4cyu3 & "everywhere" \\
\hline 隨地 & ceoi4dei6 & "everywhere" \\
\hline 前後 & cin4hau6 & "altogether" \\
\hline 大大話話 & daai6daai6waa6waa6 & "approximately" \\
\hline 大約 & daai6joek3 & "approximately" \\
\hline 大嗱嗱 & daai6laa4laa4 & "as much as" \\
\hline 大略 & daai6loek2 & "roughly" \\
\hline 大致(上) & daai6zi3(soeng6) & "roughly; approximately; more or less" \\
\hline 單係 & daan1hai6 & "only" \\
\hline 點止 & dim2zi2 & "not only" \\
\hline 頂多 & ding2do1 & "at the most" \\
\hline 頂籠 & ding2lung2 & "at the most" \\
\hline 都c & dou1 & "also; as well" \\
\hline 都 $\mathrm{d}$ & dou1 & "all" \\
\hline 到處 & dou3cyu3 & "everywhere" \\
\hline
\end{tabular}




\begin{tabular}{|c|c|c|}
\hline 加加埋埋 & gaalgaalmaai4maai4 & "altogether" \\
\hline 咁滯 & gam3zai6 & "almost" \\
\hline 幾乎 & geilfu4 & "almost" \\
\hline 極其量 & gik6kei4loeng6 & "at the most" \\
\hline 正躍呤 & ham6baang6laang6 & "all" \\
\hline 起碼 & hei2maa5 & "at least" \\
\hline 何止 & ho4zi2 & "not only" \\
\hline 一齊 & jat1cai4 & "along with all the others" \\
\hline 一共 & jatlgung6 & "altogether" \\
\hline 一概 & jat Ikoi3 & "without exception; totally" \\
\hline 一律 & jat1leot6 & "without exception" \\
\hline 又e & jau6 & $\begin{array}{l}\text { "also; expressing information of the same } \\
\text { kind" }\end{array}$ \\
\hline 亦(都) & jik6(doul) & "as well" \\
\hline 約莫 & joek 3 mok2 & "approximately" \\
\hline 拉匀 & laailwan4 & "on average" \\
\hline 另外 & ling6ngoi6 & "in addition" \\
\hline 唔只 & $m 4 z i 2$ & "not only" \\
\hline 唔淨只/唔單只 & m4zing6 zi2/m4daan 16zi2 & "not only" \\
\hline 也滯 & mat1zai6 & "not quite (negative sentence)" \\
\hline 偏偏 b & pin1pin1 & "just" \\
\hline 成 & seng4 & "full; as much as" \\
\hline 上下 & soeng6haa2 & "almost" \\
\hline 頭尾 & tau4mei5 & "from beginning to end; altogether" \\
\hline 通通 & tungltung 1 & "all; entirely" \\
\hline 唯獨是 & wai4duk6si6 & "only" \\
\hline 掙啲 & zaangldil & "almost" \\
\hline 就 $_{\mathrm{i}}$ & zau6 & "only; merely" \\
\hline 最多/至多 $\mathrm{b}$ & zeoi3dol/zi3dol & "at the most" \\
\hline 最少侄少 & zeoi3siu2/zi3siu2 & "at least" \\
\hline 只不過 & zi2batlgwo 3 & "merely; only; just" \\
\hline 只係 & zi2hai6 & "merely; only; just" \\
\hline 淨 & zing6 & "only" \\
\hline 淨係 & zing6hai6 & "only" \\
\hline 座底 & zo6dai2 & "at least" \\
\hline 將近 & zoenglgan6 & "nearly; almost" \\
\hline 再c & zoi3 & "also" \\
\hline 足足 & zuklzukl & "full; as much as" \\
\hline 總共 & zung2gung6 & "altogether" \\
\hline 仲 $c$ & zung6 & "additionally" \\
\hline
\end{tabular}

\section{Time Adverb}

Past/Present/Future Time Adverb

\begin{tabular}{|l|l|l|}
\hline 不舅 & bat1laul & "consistently; all long" \\
\hline 本來 & bun2loi4 & "originally" \\
\hline 㖵經 & cang4ging1 & "ever" \\
\hline
\end{tabular}




\begin{tabular}{|c|c|c|}
\hline 慥時 & ceoi4si4 & "at all times" \\
\hline 週啲(再) & ci4dil (zoi3) & "later" \\
\hline 遲早 & ci4zou2 & "some time in the future; some day" \\
\hline 初 & col & "at first" \\
\hline 初初 & colcol & "at the beginning" \\
\hline 初頭 & coltau4 & "at the beginning" \\
\hline 從來 & cung4loi4 & "always; at all times" \\
\hline 大早 & daai6zou2 & "some time ago" \\
\hline 第(二)日 & dai6(jib)jat6 & "later" \\
\hline 第(二)時 & dai6(ji6)si4 & "later; next time" \\
\hline 第一時間 & dai6jatlsi4gaan 3 & "immediately" \\
\hline 當初 & donglcol & "originally" \\
\hline 當堂 & dongltong4 & "immediately" \\
\hline 到時 & dou3si4 & "at that time" \\
\hline 到頭梂 & dou3tau4lai4 & "in the end; finally" \\
\hline 分分鐘 & fanlfanlzung 1 & "at any time" \\
\hline 跟手 & gan1sau2 & "next; then; after that; immediately" \\
\hline 跟住 & $\operatorname{gan} 1 z y u 6$ & "afterwards; then" \\
\hline 舊底 & gau6dai2 & "in the past" \\
\hline 經已 & ginglji5 & "already" \\
\hline 過後 & gwo3hau6 & "afterwards; later" \\
\hline 踧度㸱處 & hai2dou6/hai2syu3 & "in progress of" \\
\hline 後赶 & hau6meil & "afterwards; later" \\
\hline 起初 & hei2col & "originally" \\
\hline 起先 & hei2 $\sin 1$ & "at the beginning" \\
\hline 响度响處 & hoeng2dou6/hoeng2syu 3 & "in progress of" \\
\hline 向來 & hoeng3loi4 & "always" \\
\hline 一邊... 一邊. & jat1bin $1 \ldots$ jat 1bin $1 \ldots$ & "at the same time" \\
\hline 二便... 一便.. & jat1 bin $6 \ldots$..jat1bin $6 \ldots$ & "at the same time" \\
\hline 一齊。 & jat1cai4 & "at a time" \\
\hline 一向 & jat1hoeng3 & "consistently; all along" \\
\hline 一路 $\mathrm{a}_{\mathrm{a}} .$. 一路 $\mathrm{a} \ldots$ & jat1lou6...jat1lou6... & "at the same time" \\
\hline 一路 b & jat1lou6 & "continuously; always; all along" \\
\hline 一面... 一面.. & jat 1 min $6 \ldots$...jat $1 \min 6 \ldots$ & "at the same time" \\
\hline 一時(之間) & jat1si4(zilgaan1) & "for the time being" \\
\hline 一直 & jatlzik6 & "consistently" \\
\hline 一早 & jatlzou2 & "in advance" \\
\hline 依然 & jiljin4 & "at the same time" \\
\hline 已經 & ji5gingl & "already" \\
\hline 仍然 & jing4jin 4 & "still; yet" \\
\hline 預先 & jyu6sin 1 & "in advance; beforehand" \\
\hline 預早 & jyu6zou2 & "in advance; beforehand" \\
\hline 原先 & jyun $4 \sin 1$ & "originally" \\
\hline 立即 & laap6zik1 & "immediately" \\
\hline 連隨 & Iin 4 ceoi4 & "immediately" \\
\hline 啱(啱)先 & ngaam 1(ngaam1) $\sin 1$ & "just" \\
\hline 啱啱 $b$ & ngaam 1ngaam1 & "just" \\
\hline 新近 & sanlgan6 & "recently" \\
\hline
\end{tabular}




\begin{tabular}{|c|c|c|}
\hline 晨早 & $\operatorname{san} 4 z o u 2$ & "well in advance" \\
\hline 晨早 b & $\operatorname{san} 4 z o u 2$ & "early in the morning" \\
\hline 首先 & $\operatorname{sau} 2 \sin 1$ & "first of all" \\
\hline 試過 & si3gwo3 & "before; formerly; in the past" \\
\hline 先 $\mathrm{d}($ 至 $)$ & $\sin 1(z i 3)$ & "first" \\
\hline 先 $\mathrm{e}$ (至) & $\sin 1(z i 3)$ & "just" \\
\hline 先先/先前 & $\sin 1 \sin 1 / \sin 1 \operatorname{cin} 4$ & "before; previously" \\
\hline 素來 & sou3loi4 & "always; usually" \\
\hline 頭頭 & tau4tau2 & "at the beginning" \\
\hline 同時 & tung4si4 & "at the same time" \\
\hline 就 & zau6 & "very soon" \\
\hline 就 $\mathrm{k}$ & zau6 & "originally" \\
\hline 就快 & zau6faai3 & "very soon" \\
\hline 就嚟 & zaublai4 & "very soon" \\
\hline 正話 & zeng3waa 6 & "just" \\
\hline 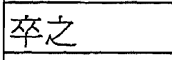 & zeot1zil & "finally" \\
\hline 即刻 & ziklhaakl & "immediately" \\
\hline 接住 & ziр3zyu6 & "immediately afterwards; in a minute" \\
\hline 將近 $b$ & zoenglgan6 & "very soon" \\
\hline 再 & $20 i 3$ & "afterwards" \\
\hline 早日 & zou2jat6 & "soon" \\
\hline (老)早就 & (lou5)zou2zau6 & "in advance" \\
\hline 早早 & zou 2 zou 2 & "well in advance" \\
\hline 終於 & zungljyuI & "finally" \\
\hline 終須 & zunglseoil & "anyhow; eventually; after all" \\
\hline 總算 ${ }_{\mathrm{b}}$ & zung $2 \operatorname{syun} 3$ & "at long last; finally" \\
\hline 仲 d & zung6 & "still; yet" \\
\hline 仲係 & zung6hai6 & "still; nevertheless" \\
\hline 轉(個)頭 & zyun3(go3)tau4 & "later" \\
\hline
\end{tabular}

Frequency

\begin{tabular}{|l|l|l|}
\hline 不斷 & bat1dyun6 & "continuously" \\
\hline 次次 & ci3ci3 & "every time" \\
\hline 動不動 & dung6bat1dung6 & "frequently" \\
\hline 凡親 & fan4can1 & "whenever" \\
\hline 逢(親) & fung4(can1) & "whenever" \\
\hline 間(唔)中 & gaan3(m4)zung1 & "occasionally" \\
\hline 久唔久 & gau2m4gau2 & "once in a while" \\
\hline 係噉 & hai6gam2 & "continuously" \\
\hline 好多時 & hou2do1si4 & "very often" \\
\hline 好日 & hou2jat6 & "rarely; seldom" \\
\hline 好少 & hou2siu2 & "rarely; seldom" \\
\hline 一日到[黑 a & jat1jat6dou3hak1 & "always" \\
\hline 一味 & jat1mei2 & "blindly; endlessly" \\
\hline 一時時 & jat1 si2si4 & "occasionally" \\
\hline 一時...一時... & jat1si4...jat1si4... & "sometimes... sometimes..." \\
\hline 日日 & jat6jat6 & "daily; regularly" \\
\hline
\end{tabular}




\begin{tabular}{|c|c|c|}
\hline 有幾何 & jau5gei2ho2 & "rarely; seldom" \\
\hline 有時 & jau5si4 & "sometimes" \\
\hline 有陣時 & jau5zan6si2 & "sometimes" \\
\hline 有啲 & jukldil & "easily; frequently" \\
\hline 有阴下 & juklhaas & "easily; frequently" \\
\hline 勤啲 & kan4dil & "frequently" \\
\hline 嗱埋 b & laa2maai4 & "easily; frequently; at every turn" \\
\hline 䊖日 & laam3jat6 & "every two days" \\
\hline 無時無刻 & mou4si4mou4hakl & "all the time (negative sentence)" \\
\hline 無幾何 & mou5gei2ho2 & "rarely; seldom" \\
\hline 無日 & mou5jat6 & "all the time (negative sentence)" \\
\hline 每 & mui5 & "whenever" \\
\hline 耐不耐/而唔耐 & noi6bat1noi2/noi6m 4 noi2 & "once in a while" \\
\hline 而唔中 & noi6m4zungl & "once in a while" \\
\hline 頻頻 & pan4pan 4 & "again and again" \\
\hline 成日 & seng $4 j a t 6$ & "always" \\
\hline 時...時... & si4...si4 ... & "sometimes...sometimes..." \\
\hline 時不時 & si4bat1si4 & "sometimes" \\
\hline 時時 & si4si4 & "often; constantly" \\
\hline 時時刻刻 & si4si4haklhakl & "all the time" \\
\hline 通常 & tungl soeng 4 & "usually" \\
\hline 枕住 & zam2zyu6 & "continuously" \\
\hline 周(不)時 & zaul(bat1)si4 & "often; constantly" \\
\hline 淨係 b & zing6hai6 & "constantly" \\
\hline 再e & $z o i 3$ & "continuously" \\
\hline 總係 & zung2hai6 & "always; invariably" \\
\hline
\end{tabular}

Duration

\begin{tabular}{|l|l|l|}
\hline 長 & coeng4 & "for a long time" \\
\hline 一度 & jat1dou6 & "for a time" \\
\hline 一日到黑 b & jatljat6dou3hak1 & "from morning till night; all day long" \\
\hline 一睴眼 & jat1zaam2ngaan5 & "in an instant" \\
\hline 日夜 & jat6je6 & "day and night" \\
\hline 雴時間 & saap3si4gaan1 & "in a twinkling" \\
\hline 永遠 & wing5jyun5 & "forever" \\
\hline 䀞下下眼 & zaam2haa5ngaan5 & "in an instant" \\
\hline 枕長 & zam2coeng4 & "for a long time" \\
\hline 轉下下眼 & zyun2haa5ngaan5 & "in an instant" \\
\hline
\end{tabular}




\section{References}

\section{English Works Cited}

Bellert, I. 1977. "On Semantic and Distributional Properties of Sentential Adverbs". Linguistic Inquiry 8(2):337-351.

Biber, D., Johansson, S., Leech, J., Conrad, S., \& Finegan, E. 1999. Longman Grammar of Spoken and Written English. England: Longman.

Burton-Roberts, N. 1991. "Prepositions, Adverbs and Adverbials" in I. Van-Ostade and J. Frankis (Eds.) Language: usage and description: studies presented to N.E. Osselton on the occasion of his retirement (p.159-172). Amsterdam and Atlanta: Rodopi.

Chafe, W. 1986. "Evidentiality in 'English conversations and academic writing" in W. Chafe and J. Nichols (Eds.) Evidentality: The Linguistic Coding of Epistemology (p.261-272). Norwood, NJ: Ablex.

Chao, Wynn, \& Mui, Evelynne Ki-Mei. 1999. The Forms of Negation in Cantonese. Paper presented at the Proceedings of the Tenth North American Conference on Chinese Linguistics. University of Southern California.

Chao, Yuen Ren. 1968. A Grammar of Spoken Chinese. Berkeley and Los Angeles: University of California Press.

Conradie, C.J. 1993. "On subjectification in modal adverbs" in H. Andersen (Ed.)

Historical Linguistics 1993: Selected papers from the $11^{\text {th }}$ International

Conference on Historical Linguistics, Los Angeles, 16-20 August 1993.

Amsterdam and Philadelphia: John Benjamins Publishing Company.

Erust, T.B. 1984. Towards an integrated theory of adverb position in English.

Bloomington: Indiana University Linguistic Club.

Greenbaum, S. 1969. Studies in English Adverbial Usage. London and New York: Longman.

Hoffner, C., Cantor, J., \& Badzinski, D. 1990. "Children's understanding of adverbs denoting degree of likelihood". Journal of Child Language 17(1):217-231.

Hoye, L. 1997. Adverbs and Modality in English. London and New York: Longman. Huang, S.F. 1975. A Study of Adverbs. Hague: Mouton.

Jackendoff, R.S. 1972. Semantic Interpretation in Generative Grammar. Cambridge, Massachusetts and London: MIT Press. 
Jacobson, S. 1978. On the Use, Meaning and Syntax of English Preverbal Adverbs. Stockholm: Almqvist \& Wiksell International.

Kjellmer, G. 1984. "Why great:greatly but not big: *bigly?". Studia Linguistica 38(1):1-19.

Klein, H. 1998. Adverbs of Degree in Dutch and Related Languages. Amsterdam and Philadelphia: John Benjamins Publishing Company.

Kryk-Kastovsky, B. 1997. "From temporal adverbs to discourse particles: An instance of cross-linguistic grammaticalisation?" in T. Nevalainen and L. Kahlas-Tarkka (Eds.) To explain the present: Studies in the changing English language in honour of Matti Rissanen (p.319-328). Helsinki: Société Néophilologique.

Kwok, H. 1984. Sentence Particles in Cantonese. Hong Kong: University of Hong Kong, Centre for Asian Studies.

Lau, W.M. 1999. A Study of Adjectives in Cantonese. Unpublished M. Phil. thesis. University of Hong Kong. Hong Kong.

Lehrer, A. 1975a. "Complement-oriented Adverbs". Linguistic Inquiry 6(3):489-494.

Lehrer, A. 1975b. "Interpreting certain adverbs: semantics or pragmatics?" Journal of Linguistics 6(2):239-248.

Li, C., \& Thompson, S. 1989. Mandarin Chinese: A Functional Reference Grammar. Berkeley and Los Angeles: University of California Press.

Luke, K.K. 1990. Utterance Particles in Cantonese Conversation. Amsterdam and Philadelphia: John Benjamins Publishing Company.

Luke, K.K., \& Nancarrow, O.T. 1997. Modal particles in Cantonese: a corpus-based study. Paper presented at the Yuen Ren Society for the Promotion of Chinese Dialect Fieldwork, March. University of Washington.

Luke, K.K., \& Nancarrow, O.T. 1998. "Auxiliary Verbs in Cantonese" in S. Matthews (Ed.) Studies in Cantonese Linguistics (p.85-100). Hong Kong: Linguistics Society of Hong Kong.

Macaulay, R. 1995. "The adverbs of authority". English World-Wide 16(1):37-60.

Matthews, S., \& Yip, V. 1994. Cantonese: A Comprehensive Grammar. London and New York: Routledge.

McConnell-Ginet, S. 1982. "Adverbs and logical form: a linguistically realistic theory". Language 58(1):144-184.

Moore, R. 1993. "Events, situations, and adverbs" in M. Bates and R. Weischedel (Eds.) Challenges in natural language processing. Cambridge: Cambridge University Press.

Nakamura, W. 1997. "A cognitive approach to English adverbs". Linguistics 35(1):247-287. 
Nancarrow, O.T., \& Luke, K.K. 1998. "The Expression of Futurity in English and Cantonese Verbs" in S. Matthews (Ed.) Studies in Cantonese Linguistics (p.101-112). Hong Kong: Linguistics Society of Hong Kong.

Peters, H. 1994. "Degree Adverbs in Early Modern English" in D. Kastovsky Studies in Early Modern English. Berlin and New York: Mouton de Gruyter.

Powell, M. 1992. "The systematic development of correlated interpersonal and metalinguistic uses in stance adverbs". Cognitive Linguistics 3(1):75-110.

Quirk, R., \& Greenbaum, S. 1973. A University Grammar of English. England: Longman.

Ramat, P, \& Ricca, D. 1998. "Sentence adverbs in the languages of Europe" in J. Van-der-Auwera and D. Ó-Baoill (Eds.) Adverbial constructions in the languages of Europe (p.187-275). Berlin and New York: Mouton De Gruyter.

Schank, R. 1974. "Adverbs and Belief". Lingua 33(1):45-67.

Swan, T. 1988. "The Development of Sentence Adverbs in English". Studia Linguistica 42(1):1-17.

Tao, Hongyin. 2000. "Adverbs of Absolute Time and Assertiveness in Vernacular Chinese: A Corpus-Based Study". Journal of the Chinese Language Teachers Association 35(2):53-74.

Thomason, R.H., \& Stalnaker, R.C. 1973. "A Semantic Theory of Adverbs". Linguistic Inquiry 4(2): 195-220.

University of Hong Kong. 2001. Notes from Research Methods in Linguistics on the topic of Dialect Survey lectured by Zhan Bohui 詹伯慧. Hong Kong: University of Hong Kong.

Valera, S. 1998. "On the subject-orientation in English -ly adverbs". English Language and Linguistics 2(2):263-282.

Vendler, Z. 1984. "Adverbs of action" in D. Testen, V. Mishra and J. Drogo (Eds.) Papers from the Parasession on Lexical Semantics (p.297-307). Chicago: Chicago Linguistic Society.

Wong, L.Y. 2001a. Sequential orders in Cantonese adverbial strings. Paper presented at the Postgraduate Research Forum on Language and Linguistics 2001, May. City University of Hong Kong.

Wong, L.Y. 2001b. The positioning of adverbs: A qualitative analysis. Paper presented at the Linguistic Society of Hong Kong Annual Research Forum 2001, December. Hong Kong Baptist University.

Wong, L.Y. 2002. On the syntactic functions of adverbs: A contrastive analysis of Cantonese, Mandarin and English. Paper presented at Bouillon De Langues Sens et structures: approche contrastive, April. Université de Lille III, France.

Wyner, A.Z. 1998. "Subject-oriented Adverbs are Thematically Dependent" in S. 
Rothstein (Ed.) Events and Grammar (p.333-348). Dordrecht, Boston, London:

Kluwer Academic Publishers. 


\section{Chinese Works Cited 中文參考書目}

Cai, Jianhua. 蔡建華 1995a 廣州話副詞的辨別 鄭定歐、潘小洛編 《廣州話研 究和教學(第二輯)》 廣州：中山大學學報編輯部

Cai, Jianhua. 蔡建華 1995b 廣州話動詞後的 “先’ 鄭定歐、潘小洛編《廣州話 研究和教學(第二輯)》 廣州 : 中山大學學報編輯部

Cai, Jianhua. 蔡建華 1997 廣州話副詞 “都’ 淺議 詹伯慧編《第五屆國際粵方 言砰討會論文集》 廣州：暨南大學出版社

Cai, JianHua. 蔡建華 1998 廣州話動詞前 “有”的詞性制別 鄭定歐、蔡建華編 《廣州話㸴究與教學(第三輯)》 廣州：中山大學出版社

Chao, Enfong. 趙恩芳 1998 談 ‘時間副詞” 和 ‘時間名詞” 《中國成人教育》 1998 年第 7 期 頁 33,38

Chen, Baoru. 陳寶如 1982 試論廣州話十一個副詞在句中的位置 《華南師院學 報(社會科學版)》 1982 年第 2 期 頁 95-99

Chen, Huiying. 陳慧英 1985 廣州話的 ‘噉” 和 ‘咁’ 《方言》1985 年第 4 期 頁 297-304

Chen, Xiaoming. 陳小明 2001 粤方言副詞 “出唪呤” 再探 論文宣讀於第八屆 國際粵方言䂰討會 十二月 廣州 暨南大學

Chen, Zijiao. 陳子䣖 1996 “都” 的語義指向 《漢語學習》1996 年第 6 期 頁 54-56

Cheng, Ting Au. 鄭定歐 1990 粵語 ‘先’ 分析 詹伯慧編 《第二屆國際粵方言 研討會論文集》 廣州：暨南大學出版社

Cheng, Ting Au. 鄭定歐編纂 1997 《香港奥語詞典》 南京 : 江蘇教育出版社

Cheung, Hung-nin Samuel. 張洪年 1972 《香港粵語語法的研究》 香港 : 香港 中文大學出版社

Chu, Zexiang and Liu, Jiesheng. 信澤祥 劉街生 1997 “細節顯現”與 ‘副+ 名'《語文建設》1997 年第6 期 頁 15-17

Ding, Shengshu, Lü, Shuxiang, Li, Rong, Sun, Dexuan, Guan, Xiechu, Fu, Jing, Huang, Chengzhang, \& Chen, Zhiwen. 丁聲樹 呂叔湘 李榮 孫德宣 管临初 傅婧 黃盛璋 陳治文 1979 《現代漢語語法講話》 北京：商務印書館

Duan, Yehui. 段業輝 1999. 語氣副詞的分布及語用功能《現代漢語語法語義碑 究》 南京：南京師範大學出版社

Guo, Chungui. 郭春貴 1997 時間副詞 ‘已經” 和 ‘都” 的異同 《世界漢語教 學》 1997 年第 2 期 頁 35-41

Han, Rongzhu. 韓容洙 2000 現代漢語的程度副詞 《漢語學習》 2000 年第 2 期 頁 12-15

He, Yang. 賀陽 1994 “程度副詞+有+名’ 試析《漢語學習》1994 年第 2 期 頁 
$22-24$

Hou, Xuechao. 侯學超編 1998 《現代漢語虛詞詞典》 北京：北京大學出版社 Jiang, Huichuan, Xu, Haoguang, Liu, Yanxin, \& Song, Fengying. 姜滙川 許皓光 劉延新 宋鳳英編 1989 《現代漢語副詞分類實用詞典》 北京：對外貿易教 育出版社

Jiang, Yan. 蔣噉 1998 語用推理與 ‘都’ 的句法語義特征 《現代外語》 1998 年第 1 期 頁 10-24

Jin, Conggao. 金琮鎬 2000 現代漢語句子副詞的語法、語義功能 陸儉明 沈陽 袁毓林編 《面臨新世紀挑戰的現代漢語語法矷究：'98 現代漢語語法學國 際學術會議論文集》濟南：山東教育出版社

Lei, Jiezhen. 雷杰珍 1999 現代漢語副名結構淺析 《廣東教育學院學報》1999 年第 4 期 頁 $41-45$

Li, Quan. 李泉 1996 副詞和副詞的再分類 胡明揚編 《詞類問題考察》北京： 北京語言學院出版社

Li, Xinkui, Huang, Jiajiao, Shi, Qisheng, Mai, Yun, \& Chen, Dingfang. 李新魁 黃 家教 施其生 麥耘 陳定方著 1995 《廣州方言㸴究》廣東：廣東人民出版 社

Liu, Yuanman. 劉元滿 1999 '太+形/動’ 與 ‘了’ 《語言教學與矿究》 1999 年第 1 期 頁 145-151

LSHK. 香港語言學學會奥語括音字表編寫小組 1997 《粵語括音字表》香港： 香港語言學學會

Lu Jianming. 陸儉明 1999 關於漢語詞類的劃分 馬慶株編 《語法研究入門》 北京：商務印書館

Lu, Jianming, \& Ma, Zhen. 陸儉明 馬真 1999 《現代漢語虛詞散論》 北京：語 文出版社

Lü, Shuxiang. 吕叔湘 1979 《漢語語法分析問題》 北京：商務印書館

Lü, Shuxiang. 吕叔湘主編 1999 《現代漢語八百詞：增訂本》 北京：商務印書 館

Luke, Kang Kwong. 陸鏡光 2002 廣州話句未 “先” 的話語分析 《暨南學報(哲 學社會科學)》 2002 年第 2 期 頁 41-44

Luo, Qingsong. 羅青松 1995 副詞 “夠’ 的語法特點分析 《殷都學刊》 1995 年第 1 期 頁 98-101

Mai, Yun. 麥耘 1993 廣州話 “先’ 再分析 鄭定歐、周小兵編 《廣州話研究和 教學(第一輯)》 廣州：中山大學出版社

Mai, Yun, \& Tan, Buyun. 麥耘 譚步雲編 1997 《實用廣州話分類詞典》廣東： 廣東人民出版社

Peng, Xiaochuan. 彭小川I 2001 廣州話的結構助詞 “噉” 論文宣讀於第八屆國 際粵方言䂰討會十二月 廣州 暨南大學 
Qiao, Yannong. 喬硯農著 1966 《廣州話口語詞的研究》香港：華僑語文出版 社

Qufu Shifan Daxue Benshu Bianxiezu. 曲阜師範大學本書編寫組編著 1992 《現 代漢語常用虛詞詞典》 杭州：浙江教育出版社

Rao, Bingcai, Ouyang, Xueya, \& Zhou, Wuji. 饒秉才 歐陽覺亞 周無忌編著 1996 《廣州話方言詞典》 香港：商務印書館

Rao, Bingcai, Ouyang, Xueya, \& Zhou, Wuji. 饒秉才 歐陽覺亞 周無忌編著 1997 《廣州話詞典》廣東 : 廣東人民出版社

Ren, Zhijing. 任芝鍈 2001 “程度副詞十名詞’ 結構的語義分析 論文宣讀於二 十一世紀首屆現代漢語語法國際碑討會(暨第七屆現代漢語語法碑討會) 二 月 香港城市大學

Shi, Xiyao. 史鍚堯 1990 副詞 “都”語義語用綜合考察 《漢語學習》1990年 第 4 期 頁 6-10

Wang, Hai. 王還主編 1992 《漢英虛詞詞典》 北京 : 華語教學出版社

Wang, Hong. 王紅 1999 副詞 ‘都’ 的語法意義分析 《漢語學習》1999 年第 6 期 頁 55-60

Wang, Li. 王力 1959 《中國現代語法(上冊)》 香港 : 中華書局

Wang, Xiaoxin and Zhang, Ge. 王小莘 張舸 1998 ‘程度副詞十名詞’ 是當前漢 語運用中値得注意的一種現象 《語言文字應用》 1998 年第 2 期 頁 42-43

Wang, Ziqiang. 王自強編著 1984 《現代虛詞用法小詞典》上海：上海辭書出 版社

Wu, Kaibun. 吳開斌 1997 《香港話詞典》廣東 : 花城出版社

Xia, Huimin. 夏慧敏 1999 試析 ‘副+名’ 《大連大學學報》1999 年第 3 期 頁 83-85

Xing, Fuyi. 刑福義 1997 “很淑女’之類說法語言文化背景的思考 《語言研 究》 1997 年第 2 期 頁 1-10

Yang, Defeng. 楊德峰 1999 副詞修飾動詞性成分形成的結構的功能 《漢語學 習》1999 年第 1 期 頁 17-20

Yang, Mansheng. 楊滿生 1998 淺析副詞 ‘都’ 《北京第二外國語學院學報》 1998 年第 2 期

Yang, Rongxiang. 楊榮洋 2000 “範圍副詞” 中的功能差異 - 兼論副詞次類的 劃分問題 《湖北大學學報(哲學社會科學版)》 2000 年第 4 期 頁 53-57

Yang, Yun. 楊雲 1999 不受程度副詞 “很” 修飾的心理動詞 《雲南教育學院學 報》1999 年第 1 期 頁 68-71

Yuan, Mingjun. 袁明軍 1999 受程度副詞修飾的述賓結構中動詞的類 南開大學 中文系《語言䂧究論叢》編委會編 《語言砰究論叢(第八輯)》天津 : 南開 大學出版社 
Zeng, Zifan. 曾子凡編著 1999 《廣州話、普通話口語詞對譯手冊(增訂本)》 香 港 : 三聯書店

Zhang, Baolin. 張寶林 1996 關聯副詞的範圍及其與連詞的區分 胡明揚編 《詞 類問題考察》 北京：北京語言學院出版社

Zhang, Liyan, \& Ni, Liehuai. 張勵研 倪列懷編著 1999 《港式廣州話詞典》 香 港 : 萬里書店出版

Zhang, Yisheng. 張誼生 2000 《現代漢語副詞研究》上海：學林出版社

Zhao, Lijiang. 趙立江 1998 '夠’ 的使用情況初步考察 《漢語學習》1998 年 第 3 期 頁 52-54

Zhongguo Shewui Kexueyuan Yuyan Yanjiusuo Cidian Bianjishi. 中國社會科學院 語言砰究所詞典編輯室 1999 《現代漢語詞典》 北京：商務印書館

Zhu, Dexi. 朱德熙 1982 《語法講義》 北京：商務印書館

Zhu, Dexi. 朱德熙 1985 《語法答問》 北京：商務印書館 